\title{
Chemistry of Tetrathiomolybdate: Aziridine Ring Opening Reactions and Facile Synthesis of Interesting Sulfur Heterocycles
}

Devarajulu Sureshkumar, Srinivasa Murthy Koutha, Srinivasan Chandrasekaran*
Department of Organic Chemistry,
Tel : + 918022932404
Indian Institute of Science,
Fax : + 918023602423
Bangalore-560 012,
e-mail : scn@orgchem.iisc.ernet.in
India.

\section{Supporting Information}

\begin{tabular}{|c|c|}
\hline Contents & Page number \\
\hline Experimental Procedures & \\
\cline { 1 - 1 } Aziridination & $\mathbf{S 3 - S 6}$ \\
\hline Tosylation & $\mathbf{S 7}-\mathbf{S 9}$ \\
\hline $\begin{array}{c}\text { Aziridine Ring Opening with } \\
\text { tetrathiomolybdate 1 }\end{array}$ & $\mathbf{S 9}-\mathbf{S 2 2}$ \\
\hline H $/{ }^{13}$ C NMR Spectra & \\
\hline Aziridines & $\mathbf{S 2 3}-\mathbf{S 3 0}$ \\
\hline N-Tosyl & \\
\hline Aziridine Tosylates & $\mathbf{S 3 1}-\mathbf{S 3 7}$ \\
\hline Amino Sulfides & $\mathbf{S 3 8}-\mathbf{S 6 1}$ \\
\hline
\end{tabular}




\section{Experimental}

General Procedures. All reactions were carried out in oven-dried apparatus using dry solvents under anhydrous conditions, unless otherwise noted. Reaction mixtures were stirred magnetically unless otherwise stated. Commercial grade solvents were distilled and dried according to literature procedures ("Purification of laboratory chemicals", $3^{\text {rd }}$ Ed., D. D. Perrin, W. L. F. Armarego, Pergamon Press, Oxford, 1988). Analytical TLC was performed on commercial plates coated with silica gel $\mathrm{GF}_{254}(0.25$ mm). Silica gel (230 - 400 mesh) was used for column chromatography. Melting points determined are uncorrected. Yields refer to chromatographically and spectroscopically ( ${ }^{1}$ H NMR) homogeneous materials, unless otherwise stated. NMR spectra were recorded on 300 or $400 \mathrm{MHz}$ instruments and calibrated using residual undeuterated solvent as an internal reference. The following abbreviations explain the multiplicity $\mathrm{s}=$ singlet, $\mathrm{d}=$ doublet, $\mathrm{t}=$ triplet, $\mathrm{q}=$ quartet, $\mathrm{m}=$ multiplet, $\mathrm{br}=$ broad. IR spectra were recorded on a FT-IR spectrometer. High-resolution mass spectra (HR-MS) were recorded on a Micromass Q-TOF mass spectrometer.

Preparation of ammonium tetrathiomolybdate ${ }^{1}\left[\mathrm{NH}_{4}\right]_{2} \mathrm{MoS}_{4}$ : Ammonium molybdate $(10 \mathrm{~g})$ was dissolved in a mixture of ammonium hydroxide $(60 \mathrm{~mL})$ water $(20 \mathrm{~mL})$, and the solution was filtered. Hydrogen sulfide was bubbled rapidly at ambient temperature into the solution mixture until it was saturated and then the temperature was raised to $60{ }^{\circ} \mathrm{C}$ while maintaining a slow stream of hydrogen sulfide. After $60 \mathrm{~min}$, the mixture was cooled to $0{ }^{\circ} \mathrm{C}$ for 30 min and the product was isolated by filtration. The crystalline 
solid was washed with isopropyl alcohol $(25 \mathrm{~mL})$ and ether $(25 \mathrm{~mL})$ and dried under vacuum to obtain brick red crystals of ammonium molybdate (13.4 - $14.2 \mathrm{~g}, 92 \%)$.

$\mathrm{UV}-\mathrm{Vis}\left(\mathrm{H}_{2} \mathrm{O}\right): \lambda_{\max }(\mathrm{nm}),(\varepsilon): 472(11,850) ; 326 \quad(16,750) ; 241 \quad(24,700)$.

IR (KBr): 750, 700, 525, 450 .

Preparation of Benzyltriethylammonium tetrathiomolybdate ${ }^{2}\left[\mathrm{BnEt}_{3} \mathrm{~N}_{2} \mathrm{MoS}_{4}\right.$ : A solution of benzyltriethylammonium chloride $(23.3 \mathrm{~g}, 102.5 \mathrm{mmol})$ in distilled water (60 $\mathrm{mL}$ ) was added to a well stirred solution of ammonium tetrathiomolybdate (13 $\mathrm{g}, 50$ $\mathrm{mmol})$ in distilled water $(60 \mathrm{~mL})$. Vigorous stirring was continued for $2 \mathrm{~h}$ at room temperature and the solid was filtered and washed with isopropyl alcohol $(40 \mathrm{~mL})$ and diethylether $(40 \mathrm{~mL})$. After drying at high vacuum, brick red powder of benzyltriethylammonium tetrathiomolybdate 1 was stored in a desiccator ( $24 \mathrm{~g}, 80 \%)$.

mp: $150{ }^{\circ} \mathrm{C}$ (decomp). UV - Vis (DMF): $\lambda_{\max }(\mathrm{nm}),(\varepsilon): 472(11,850) ; 320$ (16,750);

274 (24,700); Anal. Calcd for $\left(\mathrm{C}_{26} \mathrm{H}_{44} \mathrm{~N}_{2} \mathrm{~S}_{4} \mathrm{Mo}\right) \mathrm{C}, 51.29 ; \mathrm{H}, 7.28 ; \mathrm{N}, 4.60$. Found C, 50.93; H, 7.31; N, 4.31.

General procedure for the synthesis of aziridines $^{3}$. To a mixture of an appropriate olefin (3 mmol) and $\mathrm{TsNCINa} .3 \mathrm{H}_{2} \mathrm{O}(\mathrm{CAT})(0.930 \mathrm{~g}, 3.3 \mathrm{mmol})$ in $15 \mathrm{~mL}$ of $\mathrm{CH}_{3} \mathrm{CN}$, was added phenyltrimethylammonium tribromide (PTAB) $(0.113 \mathrm{~g}, 0.3 \mathrm{mmol})$ at $28{ }^{\circ} \mathrm{C}$. After $12 \mathrm{~h}$ of vigorous stirring, the reaction mixture was concentrated and filtered through a short column of silica gel and eluted with $10 \%$ EtOAc in hexanes. After solvent evaporation of solvent, the resultant solid was purified by flash column chromatography to yield the corresponding aziridine.

Scheme S1.Synthesis of cis-N-tosyl aziridine 2.

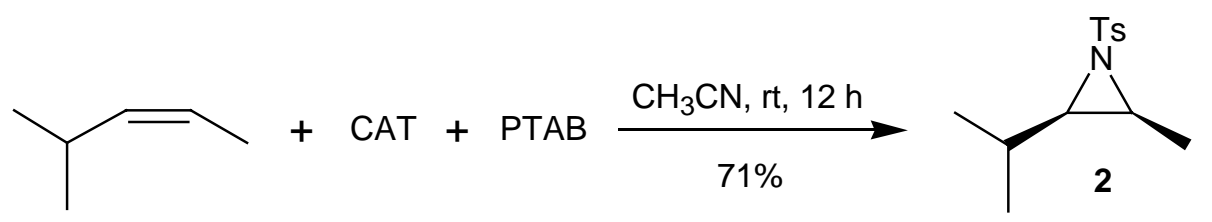


cis-N-tosyl aziridine 2: $\mathrm{R}_{\mathrm{f}}=0.45$ (EtOAc/hexanes, $3: 7$ ); Yield: $0.540 \mathrm{~g}, 71 \%$; m. p: $78{ }^{\circ} \mathrm{C}$; IR (neat) $\mathrm{V}_{\max }: 1322,1159,1091,945,868,739,671 \mathrm{~cm}^{-1} ;{ }^{1} \mathrm{H}$ NMR (300 MHz, $\left.\mathrm{CDCl}_{3}\right): \delta 7.82(\mathrm{~d}, J=8.4 \mathrm{~Hz}, 2 \mathrm{H}), 7.32(\mathrm{~d}, J=8.4 \mathrm{~Hz}, 2 \mathrm{H}), 2.94(\mathrm{~m}, 1 \mathrm{H})$, $2.43(\mathrm{~m}, 1 \mathrm{H}), 2.42(\mathrm{dd}, J=9.9,6.6 \mathrm{~Hz}, 1 \mathrm{H}), 1.44(\mathrm{~s}, 3 \mathrm{H}), 1.23(\mathrm{~d}, J=6.6 \mathrm{~Hz}, 3 \mathrm{H}), 0.92$ $(\mathrm{d}, J=6.6 \mathrm{~Hz}, 3 \mathrm{H}), 0.78(\mathrm{~d}, J=6.6 \mathrm{~Hz}, 3 \mathrm{H}) ;{ }^{13} \mathrm{C} \mathrm{NMR}\left(75 \mathrm{MHz}, \mathrm{CDCl}_{3}\right): \delta 144.1,135.4$, $129.4,127.9,51.4,40.2,26.3,21.5,20.7,19.1,11.9 ; \mathrm{HR}-\mathrm{MS} \mathrm{m} / \mathrm{z}$ : calcd for $\mathrm{C}_{13} \mathrm{H}_{19} \mathrm{NO}_{2} \mathrm{SNa}^{+}\left[\mathrm{M}+\mathrm{Na}^{+}\right]: 276.1034$; found: 276.1037 .

Scheme S2. Synthesis of trans-N-tosyl aziridine 4.

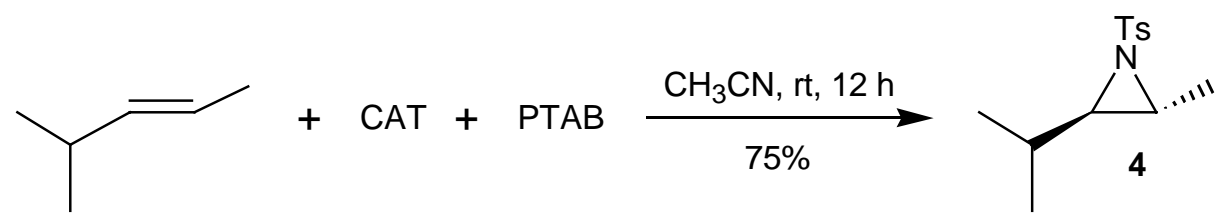

Trans-N-tosyl aziridine 4: $\mathrm{R}_{\mathrm{f}}=0.70$ (EtOAc/hexanes, $\left.3: 7\right)$; Yield: $0.570 \mathrm{~g}$, 75\%; m. p: $70{ }^{\circ} \mathrm{C}$; IR (neat) $\mathrm{V}_{\max }$ : 1321, 1158, 1089, 877, $684 \mathrm{~cm}^{-1}$; ${ }^{1} \mathrm{H}$ NMR (300 MHz, $\left.\mathrm{CDCl}_{3}\right): \delta 7.84(\mathrm{~d}, J=8.4 \mathrm{~Hz}, 2 \mathrm{H}), 7.30(\mathrm{~d}, J=8.4 \mathrm{~Hz}, 2 \mathrm{H}), 2.68(\mathrm{~m}, 1 \mathrm{H}), 2.54$ (dd, $J=6.3,3.9 \mathrm{~Hz}, 1 \mathrm{H}), 2.42(\mathrm{~s}, 3 \mathrm{H}), 1.63(\mathrm{~d}, J=6.3 \mathrm{~Hz}, 3 \mathrm{H}), 1.47(\mathrm{~m}, 1 \mathrm{H}), 0.88$ (d, $J=6.3 \mathrm{~Hz}, 3 \mathrm{H}), 0.73(\mathrm{~d}, J=6.3 \mathrm{~Hz}, 3 \mathrm{H}) ;{ }^{13} \mathrm{C} \mathrm{NMR}\left(75 \mathrm{MHz}, \mathrm{CDCl}_{3}\right): \delta 143.6,137.9$, $129.3,127.5,54.9,45.3,30.0,21.5,19.8,19.3,14.2 ; \mathrm{HR}-\mathrm{MS} \mathrm{m} / \mathrm{z}$ : calcd for $\mathrm{C}_{13} \mathrm{H}_{19} \mathrm{NO}_{2} \mathrm{SNa}^{+}\left[\mathrm{M}+\mathrm{Na}^{+}\right]: 276.1034$; found: 276.1040 .

Scheme S3. Synthesis of N-tosyl aziridine methanol:

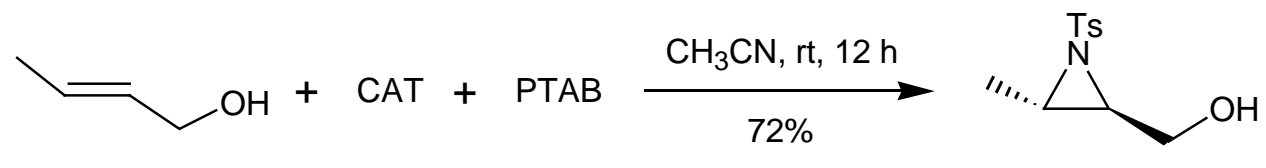

N-Tosyl aziridine methanol: $R_{\mathrm{f}}=0.60$ (silica gel, EtOAc/hexanes, $1: 1$ ); Yield: $0.520 \mathrm{~g}, 72 \%$; IR (neat) $\mathrm{V}_{\max }: 3507,1318,1159,1091,1050,937,710,686 \mathrm{~cm}^{-1}$; 
${ }^{1} \mathrm{H}$ NMR (300 MHz, $\left.\mathrm{CDCl}_{3}\right): \delta ; 7.83(\mathrm{~d}, \mathrm{~J}=8.4 \mathrm{~Hz}, 2 \mathrm{H}), 7.32(\mathrm{~d}, \mathrm{~J}=8.4 \mathrm{~Hz}, 2 \mathrm{H}), 3.96$ (m, 1H), $3.76(\mathrm{~m}, 1 \mathrm{H}), 2.96(\mathrm{~m}, 2 \mathrm{H}), 2.50(\mathrm{t}, \mathrm{J}=7.2 \mathrm{~Hz}, 1 \mathrm{H}), 2.43(\mathrm{~s}, 3 \mathrm{H}), 1.44$ $(\mathrm{d}, \mathrm{J}=5.1 \mathrm{~Hz}, 3 \mathrm{H}) ;{ }^{13} \mathrm{C} \mathrm{NMR}\left(75 \mathrm{MHz}, \mathrm{CDCl}_{3}\right): \delta 144.1,137.4,129.6,127.0,60.8,51.0$, 42.3, 21.5, 14.9; $\mathrm{HR}$ - MS.m/z: calcd for $\mathrm{C}_{12} \mathrm{H}_{17} \mathrm{NO}_{3} \mathrm{SNa}^{+}\left[\mathrm{M}+\mathrm{Na}^{+}\right]$: 278.0827; found: 278.0836 .

Scheme S4. Synthesis of N-tosyl homo aziridine methanol:

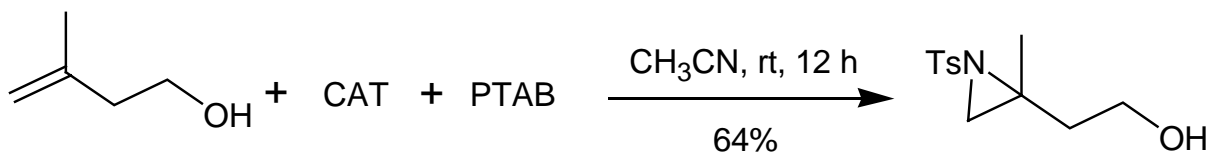

N-Tosyl homo aziridine methanol: $\mathrm{R}_{\mathrm{f}}=0.65$ (silica gel, EtOAc/hexanes, $1: 1$ ); Yield: $0.489 \mathrm{~g}, 64 \%$; IR (neat) $\mathrm{V}_{\max }: 3525,1597,1318,1158,943,818,709 \mathrm{~cm}^{-1}$; ${ }^{1} \mathrm{H}$ NMR (300 MHz, $\left.\mathrm{CDCl}_{3}\right): \delta 7.81(\mathrm{~d}, \mathrm{~J}=8.3 \mathrm{~Hz}, 2 \mathrm{H}), 7.32(\mathrm{~d}, \mathrm{~J}=8.3 \mathrm{~Hz}, 2 \mathrm{H}), 3.78$ (m, 2H), $2.61(\mathrm{~s}, 1 \mathrm{H}), 2.43(\mathrm{~s}, 3 \mathrm{H}), 2.38(\mathrm{~s}, 1 \mathrm{H}), 2.03(\mathrm{~m}, 1 \mathrm{H}), 1.76(\mathrm{~m}, 1 \mathrm{H}), 1.72(\mathrm{~s}, 3 \mathrm{H})$; ${ }^{13} \mathrm{C}$ NMR $\left(75 \mathrm{MHz}, \mathrm{CDCl}_{3}\right): \delta 144.0,137.4,129.5,127.2,59.2,48.8,40.9,39.5,21.5$, 18.9; HR - MS.m/z: calcd for $\mathrm{C}_{12} \mathrm{H}_{17} \mathrm{NO}_{3} \mathrm{SNa}^{+}\left[\mathrm{M}+\mathrm{Na}^{+}\right]$: 278.0827; found: 278.0836.

Scheme S5. Synthesis of carvone derived aziridine 16:

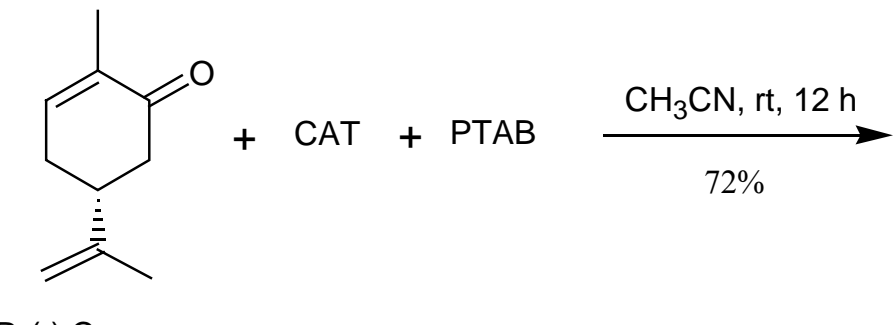

R-(-) Carvone

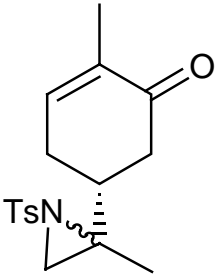

16

Aziridine derivative 16: $\mathrm{R}_{\mathrm{f}}=0.40$ (EtOAc/hexanes, $3: 7$ ); Yield: $0.689 \mathrm{~g}, 72 \%$; IR (neat) $\mathrm{V}_{\max }: 1671,1451,1320,1159,959,847,713,696 \mathrm{~cm}^{-1} ;{ }^{1} \mathrm{H}$ NMR $(300 \mathrm{MHz}$, $\mathrm{CDCl}_{3,1} 1: 1$ mixture of diastereomers): $\delta 7.81(\mathrm{~d}, J=8.4 \mathrm{~Hz}, 2 \mathrm{H}), 7.32(\mathrm{~d}, J=8.4 \mathrm{~Hz}, 2 \mathrm{H})$, $6.72(\mathrm{bs}, 1 \mathrm{H}), 2.63(\mathrm{~s}, 1 \mathrm{H}), 2.44(\mathrm{~s}, 3 \mathrm{H}), 2.28(\mathrm{~m}, 3 \mathrm{H}), 2.17(\mathrm{~s}, 2 \mathrm{H}), 1.76(\mathrm{~s}, 3 \mathrm{H}), 1.74$ 
(s, 3H), 1.69 (s, 1H); ${ }^{13} \mathrm{C}$ NMR (75 MHz, $\mathrm{CDCl}_{3}, 1: 1$ mixture of diastereomers): $\delta 198.5$, 198.4, 144.1, 144.0, 143.9, 143.6, 137.6, 137.5, 135.5, 135.3, 129.4, 127.2, 51.4, 51.1, 42.8, 41.9, 40.2, 40.1, 39.6, 39.5, 28.2, 27.9, 21.5, 15.8, 15.5, 15.0; HR - MS m/z: calcd for $\mathrm{C}_{17} \mathrm{H}_{21} \mathrm{NO}_{3} \mathrm{SNa}^{+}\left[\mathrm{M}+\mathrm{Na}^{+}\right]$: 342.1140; found: 342.1145 .

Scheme S6. Synthesis of aziridino-epoxy-carvone 21:

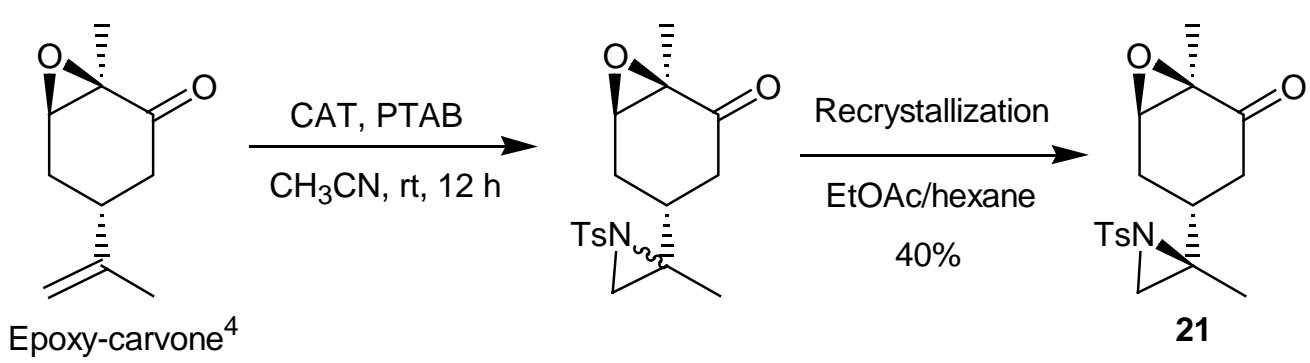

After flash chromatography the diastereomeric mixture was recrystallized using EtOAc and hexanes to afford aziridino-epoxy-carvone $\mathbf{2 1}$ as colorless crystals. $\mathrm{R}_{\mathrm{f}}=0.50$ (EtOAc/hexanes, 3 : 7); Yield: 0. 402 g, 40\%; m. p: $121{ }^{\circ} \mathrm{C} ;[\alpha]^{26}{ }_{\mathrm{D}}=+48.00(\mathrm{c}=1.0$, $\mathrm{CHCl}_{3}$ ); IR (neat) $\mathrm{V}_{\max }: 1708,1319,1159,846,712 \mathrm{~cm}^{-1} ;{ }^{1} \mathrm{H} \mathrm{NMR}\left(300 \mathrm{MHz}, \mathrm{CDCl}_{3}\right.$ ): $\delta 7.81(\mathrm{~d}, J=8.4 \mathrm{~Hz}, 2 \mathrm{H}), 7.32(\mathrm{~d}, J=8.4 \mathrm{~Hz}, 2 \mathrm{H}), 3.42(\mathrm{~d}, J=3.3 \mathrm{~Hz}, 1 \mathrm{H}), 2.63(\mathrm{~s}, 1 \mathrm{H})$, 2.57-2.50 (m, 1H), $2.44(\mathrm{~s}, 3 \mathrm{H}), 2.36-2.29(\mathrm{~m}, 1 \mathrm{H}), 2.24(\mathrm{~s}, 1 \mathrm{H}), 2.13-1.95(\mathrm{~m}, 2 \mathrm{H})$, 1.88-1.79 (m, 1H), $1.66(\mathrm{~s}, 3 \mathrm{H}), 1.40(\mathrm{~s}, 3 \mathrm{H}) ;{ }^{13} \mathrm{C} \mathrm{NMR}\left(75 \mathrm{MHz}, \mathrm{CDCl}_{3}\right): \delta 204.2$, $144.0,137.5,129.5,127.3,60.7,58.7,50.9,40.3,39.0,35.3,25.5,21.5,15.2,15.1$; HR - MS m/z: calcd for $\mathrm{C}_{17} \mathrm{H}_{21} \mathrm{NO}_{4} \mathrm{SNa}^{+}\left[\mathrm{M}+\mathrm{Na}^{+}\right]$: 358.1089; found: 358.1098 .

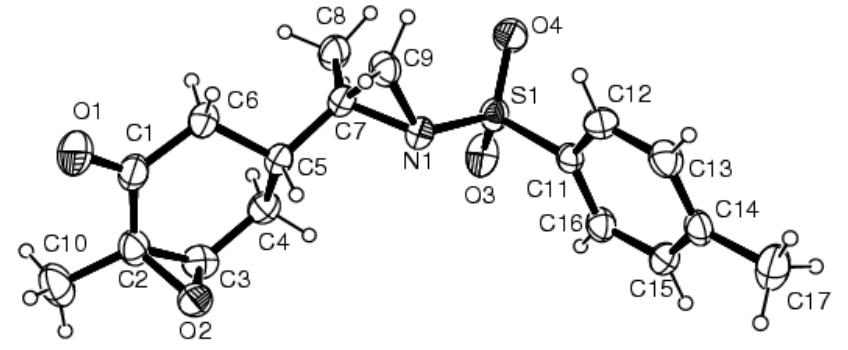

Crystal structure of compound 21

Crystal data of compound 21
$\begin{array}{ll}\mathrm{a}=8.4144(5) & \alpha=90.000 \\ \mathrm{~b}=13.3381(8) & \beta=90.000 \\ \mathrm{c}=15.0488(9) & \gamma=90.000 \\ \mathrm{~V}=1688.96(2) & \\ \text { Space group }=\mathrm{P} 2{ }_{1} 2_{1} 2_{1}\end{array}$


General procedure for the tosylation of hydroxy group of N-tosyl aziridine derivatives $^{5}$. To a solution of an appropriate aziridino-alcohol $(0.68 \mathrm{mmol})$ in $\mathrm{CH}_{2} \mathrm{Cl}_{2}$ (2 $\mathrm{mL})$ cooled to $-15{ }^{\circ} \mathrm{C}$, was added pyridine $(0.17 \mathrm{~mL}, 2.1 \mathrm{mmol})$, DMAP (10 $\mathrm{mg}$, $0.08 \mathrm{mmol})$ and $\mathrm{p}$-toluenesulfonyl chloride $(0.270 \mathrm{~g}, 1.4 \mathrm{mmol})$. After stirring for $24 \mathrm{~h}$ at $-15{ }^{\circ} \mathrm{C}$, the solution was diluted with ether $(150 \mathrm{~mL})$ and washed with water, $1 \mathrm{M} \mathrm{HCl}$, saturated $\mathrm{NaHCO}_{3}$ and water. The organic phase was dried over $\mathrm{MgSO}_{4}$ and concentrated in vacuo. Chromatography (20\% EtOAc/hexanes) gave the corresponding tosylates 6, 9 and 11 in high purity.

Scheme S7. Synthesis of trans-N-tosyl aziridinemethanol tosylate 6.
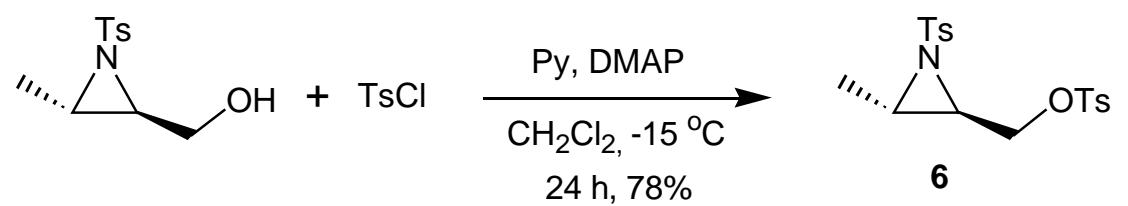

trans-N-tosyl aziridinemethanol tosylate 6: $\mathrm{R}_{\mathrm{f}}=0.65($ EtOAc/hexanes, $3: 7)$; Yield: 0.210 g, 78\%; IR (neat) $\mathrm{V}_{\max }: 1597,1495,1453,1361,1324,1177,1092,970$, 815, 709, 685, $664 \mathrm{~cm}^{-1} ;{ }^{1} \mathrm{H}$ NMR $\left(300 \mathrm{MHz} \mathrm{CDCl}_{3}\right): \delta 7.79(\mathrm{~d}, J=8.4 \mathrm{~Hz}, 2 \mathrm{H}), 7.64$ $(\mathrm{d}, J=8.4 \mathrm{~Hz}, 2 \mathrm{H}), 7.30(\mathrm{~d}, J=8.4 \mathrm{~Hz}, 4 \mathrm{H}), 4.17(\mathrm{dd}, J=11.0,5.7 \mathrm{~Hz}, 1 \mathrm{H}), 3.96$ (dd, $J=11.0,6.3 \mathrm{~Hz}, 1 \mathrm{H}), 3.01(\mathrm{~m}, 1 \mathrm{H}), 2.75(\mathrm{~m}, 1 \mathrm{H}), 2.44(\mathrm{~s}, 6 \mathrm{H}), 1.56(\mathrm{~d}, J=6.3 \mathrm{~Hz}$, $3 \mathrm{H}) ;{ }^{13} \mathrm{C}$ NMR $\left(75 \mathrm{MHz}, \mathrm{CDCl}_{3}\right): \delta 145.1,144.3,137.0,132.4,129.8,129.5,127.8$, 127.4, 68.4, 45.0, 43.6, 21.7, 21.6, 14.1; HR - MS.m/z: calcd for $\mathrm{C}_{18} \mathrm{H}_{21} \mathrm{NO}_{5} \mathrm{~S}_{2} \mathrm{Na}^{+}\left[\mathrm{M}+\mathrm{Na}^{+}\right.$]: 418.0759; found: 418.0768 .

Scheme S8. Synthesis of N-tosyl homo aziridinemethanol tosylate 9.

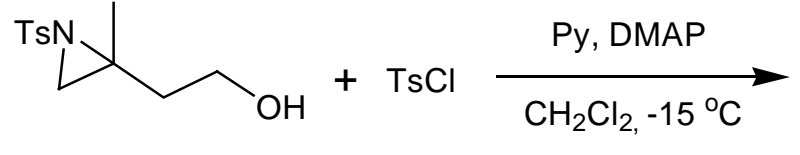

$24 \mathrm{~h}, 73 \%$

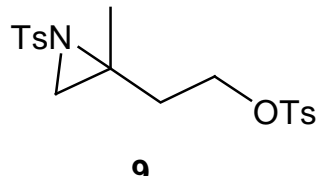

9 
N-Tosyl homo aziridinemethanol tosylate 9: $R_{f}=0.50$ (EtOAc/hexanes, $3: 7$ ); Yield: $0.205 \mathrm{~g}, 73 \%$; IR (neat) $\mathrm{V}_{\max }$ : 1597, 1358, 1320, 1176, $1159816,663 \mathrm{~cm}^{-1} ;{ }^{1} \mathrm{H}$ NMR (300 MHz, $\left.\mathrm{CDCl}_{3}\right): \delta 7.77(\mathrm{~d}, J=8.4 \mathrm{~Hz}, 4 \mathrm{H}), 7.34(\mathrm{~d}, J=8.4 \mathrm{~Hz}, 2 \mathrm{H}), 7.30$ (d, $J=8.4 \mathrm{~Hz}, 2 \mathrm{H}), 4.15(\mathrm{~m}, 2 \mathrm{H}), 2.54(\mathrm{~s}, 1 \mathrm{H}), 2.45(\mathrm{~s}, 3 \mathrm{H}), 2.43(\mathrm{~s}, 3 \mathrm{H}), 2.33(\mathrm{~s}, 1 \mathrm{H})$, $2.10(\mathrm{~m}, 1 \mathrm{H}), 1.96(\mathrm{~m}, 1 \mathrm{H}), 1.59(\mathrm{~s}, 3 \mathrm{H}) ;{ }^{13} \mathrm{C} \mathrm{NMR}\left(75 \mathrm{MHz}, \mathrm{CDCl}_{3}\right): \delta 144.9,143.9$, $137.4,132.6,129.8,129.4,127.7,127.2,66.9,47.3,41.0,36.4,21.5,21.4,18.8$ HR - MS m/z: calcd for $\mathrm{C}_{19} \mathrm{H}_{23} \mathrm{NO}_{5} \mathrm{~S}_{2} \mathrm{Na}^{+}\left[\mathrm{M}+\mathrm{Na}^{+}\right]$: 432.0915;.found: 432.0919 .

\section{Scheme S9. Synthesis of ribonolactone derived tosylate 11.}

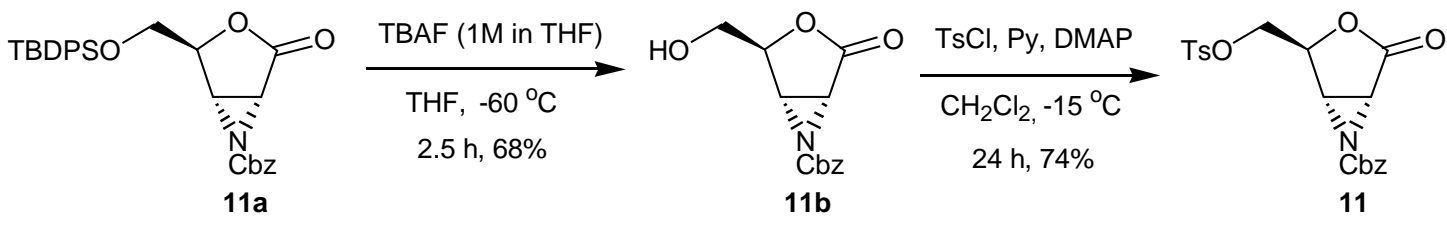

To a solution of $11 \mathrm{a}^{6}(0.280 \mathrm{~g}, 0.56 \mathrm{mmol})$ in THF $(1.6 \mathrm{~mL})$ held at $-60{ }^{\circ} \mathrm{C}$ under argon, was added a solution of TBAF $(1.0 \mathrm{M})$ in THF $(0.56 \mathrm{~mL}, 1$ equiv). After stirring for $30 \mathrm{~min}$ at $-60{ }^{\circ} \mathrm{C}$, the reaction mixture was gradually allowed to attain room temperature over $2 \mathrm{~h}$. It was then diluted with EtOAc $(0.56 \mathrm{~mL})$ and washed with water $(2 \times 10 \mathrm{~mL})$. The organic phase was dried over $\mathrm{MgSO}_{4}$ and evaporated to dryness. The resultant oily residue was purified by flash chromatography on silica gel (EtOAc/hexanes, $3: 7$ ) to afford compound $\mathbf{1 1 b}$ as a colorless oil. 11b: $\mathrm{R}_{\mathrm{f}}=0.30$ (EtOAc/hexanes, 3:7); Yield: $0.101 \mathrm{~g}, 68 \%$; IR (neat) $\mathrm{V}_{\max }: \mathrm{cm}^{-1}$; ${ }^{1} \mathrm{H}$ NMR $(300 \mathrm{MHz}$, $\left.\mathrm{CDCl}_{3}\right): \delta 7.34(\mathrm{~m}, 5 \mathrm{H}), 5.15(\mathrm{~d}, \mathrm{~J}=2.7 \mathrm{~Hz}, 2 \mathrm{H}), 4.71(\mathrm{t}, \mathrm{J}=2.4 \mathrm{~Hz}, 1 \mathrm{H}), 3.95$ (bd, J=12.9 Hz, 1H), $3.77(b d, J=12.9 \mathrm{~Hz}, 1 \mathrm{H}), 3.61(\mathrm{~d}, \mathrm{~J}=3.3 \mathrm{~Hz}, 1 \mathrm{H}), 3.48$ (d, J= 3.3 Hz, 1H), 2.99 (bs, 1H); ${ }^{13} \mathrm{C}$ NMR (75 MHz, $\mathrm{CDCl}_{3}$ ): $\delta$ 169.6, 158.3, 134.5, 128.6, 128.5, 128.4, 71.1, 69.2, 62.0, 42.1, 37.6; HR - MS m/z: calcd for $\mathrm{C}_{13} \mathrm{H}_{13} \mathrm{NO}_{5} \mathrm{Na}^{+}\left[\mathrm{M}+\mathrm{Na}^{+}\right]:$286.0691; found: 286.0702 . 
Tosylate 11: $R_{\mathrm{f}}=0.50$ (EtOAc/hexanes, $3: 7$ ); Yield: $0.210 \mathrm{~g}, 74 \%$; IR (neat) $\mathrm{V}_{\max }: 1790,1708,1530,1263 \mathrm{~cm}^{-1} ;{ }^{1} \mathrm{H}$ NMR $\left(300 \mathrm{MHz}, \mathrm{CDCl}_{3}\right): \delta 7.75(\mathrm{~d}, J=8.4 \mathrm{~Hz}$, 2H), $7.34(\mathrm{~m}, 7 \mathrm{H}), 5.13(\mathrm{dd}, J=22.5,11.7 \mathrm{~Hz}, 2 \mathrm{H}), 4.81(\mathrm{t}, J=3.0 \mathrm{~Hz}, 1 \mathrm{H}), 4.31$ (dd, $J=11.5,3.0 \mathrm{~Hz}, 1 \mathrm{H}), 4.19$ (dd, $J=11.5,3.3 \mathrm{~Hz}, 1 \mathrm{H}), 3.64(\mathrm{~d}, J=3.3 \mathrm{~Hz}, 1 \mathrm{H}), 3.49$ $(\mathrm{d}, J=3.3 \mathrm{~Hz}, 1 \mathrm{H}), 2.45(\mathrm{~s}, 3 \mathrm{H}) ;{ }^{13} \mathrm{C} \mathrm{NMR}\left(75 \mathrm{MHz}, \mathrm{CDCl}_{3}\right): \delta 167.5,157.9,145.7$, $134.4,131.6,130.1,128.7,128.6,128.4,127.9,73.4,69.4,67.7,41.4,37.3,21.6$; HR - MS m/z: calcd for $\mathrm{C}_{20} \mathrm{H}_{19} \mathrm{NO}_{7} \mathrm{SNa}^{+}\left[\mathrm{M}+\mathrm{Na}^{+}\right]$: 440.0780; found: 440.0795 .

General procedure for the reaction of simple N-tosyl aziridines with benzyltriethylammonium tetrathiomolybdate 1 . To a well-stirred solution of appropriate aziridines $(0.40 \mathrm{mmol})$ in $\mathrm{CH}_{3} \mathrm{CN} \quad(6 \quad \mathrm{~mL})$ was added benzyltriethylammonium tetrathiomolybdate $1(0.264 \mathrm{~g}, 0.43 \mathrm{mmol})$ at once and stirred at room temperature $\left(28^{\circ} \mathrm{C}\right)$ for $10 \mathrm{~h}$. The solvent was evaporated under reduced pressure and the black residue was extracted with $\mathrm{CH}_{2} \mathrm{Cl}_{2}: \mathrm{Et}_{2} \mathrm{O}(1: 5,3 \times 10 \mathrm{~mL})$ and was filtered through a Celite pad. The filtrate was concentrated and the residue was purified by flash column chromatography on silica gel to give $\beta$-aminodisulfide as white solid.

Scheme S10.Synthesis of anti- $\beta$-aminodisulfide 3.

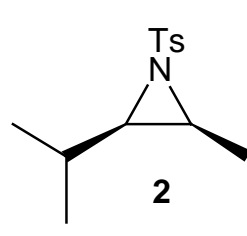

2

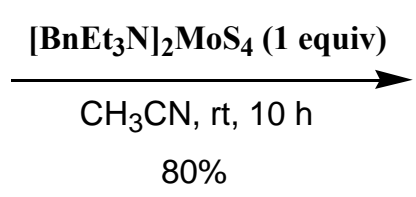

$80 \%$

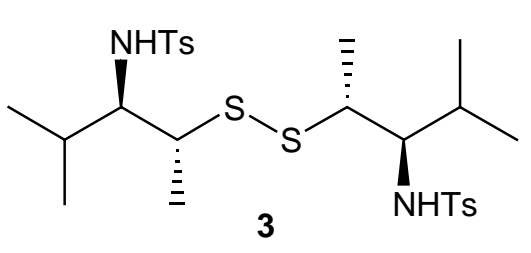

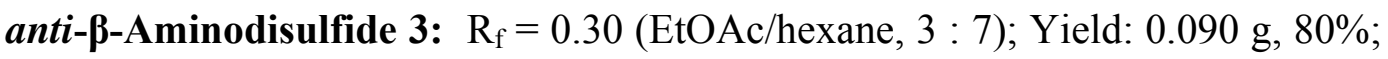
$667 \mathrm{~cm}^{-1} ;{ }^{1} \mathrm{H}$ NMR $\left(300 \mathrm{MHz}, \mathrm{CDCl}_{3}, 1: 1\right.$ mixture of diastereomers): $\delta 7.78$ (d, $J=8.4 \mathrm{~Hz}, 2 \mathrm{H}), 7.76(\mathrm{~d}, J=8.4 \mathrm{~Hz}, 2 \mathrm{H}), 7.28(\mathrm{~d}, J=8.4 \mathrm{~Hz}, 4 \mathrm{H}), 5.09(\mathrm{~d}, J=9.3 \mathrm{~Hz}$, 
1H), $4.72(\mathrm{~d}, J=9.3 \mathrm{~Hz}, 1 \mathrm{H}), 3.25(\mathrm{~m}, 2 \mathrm{H}), 3.16(\mathrm{~m}, 2 \mathrm{H}), 2.41(\mathrm{~s}, 6 \mathrm{H}), 1.92(\mathrm{~m}, 2 \mathrm{H})$, $1.31(\mathrm{~d}, J=6.6 \mathrm{~Hz}, 3 \mathrm{H}), 1.19(\mathrm{~d}, J=7.8 \mathrm{~Hz}, 3 \mathrm{H}), 0.83(\mathrm{~d}, J=7.8 \mathrm{~Hz}, 3 \mathrm{H}), 0.78$ $(\mathrm{d}, J=6.6 \mathrm{~Hz}, 6 \mathrm{H}), 0.71(\mathrm{~d}, J=6.6 \mathrm{~Hz}, 3 \mathrm{H}) ;{ }^{13} \mathrm{C} \mathrm{NMR}\left(75 \mathrm{MHz}, \mathrm{CDCl}_{3}, 1: 1\right.$ mixture of diastereomers): $\delta 143.1,143.0,138.7,138.6,129.4,126.9,63.8,63.7,49.7,30.2,21.5$, 20.9, 18.9, 18.3; HR - MS m/z: calcd for $\mathrm{C}_{26} \mathrm{H}_{40} \mathrm{~N}_{2} \mathrm{O}_{4} \mathrm{~S}_{4} \mathrm{Na}^{+}\left[\mathrm{M}+\mathrm{Na}^{+}\right]$: 595.1769; found: 595.1776.

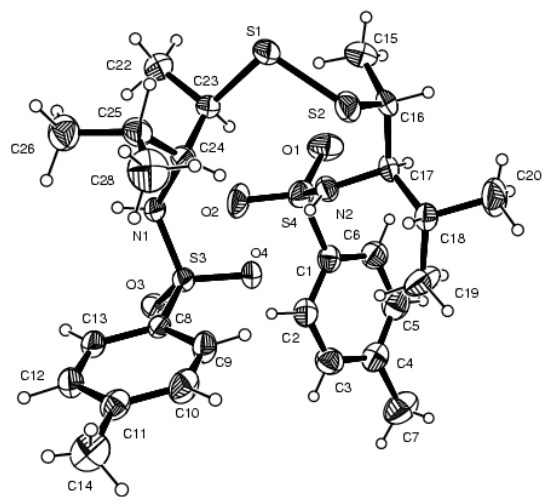

$$
\begin{aligned}
& \text { Crystal data of compound } 3 \\
& \mathrm{a}=8.8750(16) \quad \alpha=82.465(4) \\
& \mathrm{b}=11.6119(22) \quad \beta=86.737(4) \\
& \mathrm{c}=15.4880(28) \quad \gamma=74.056(4) \\
& \mathrm{V}=1521.11(20) \\
& \text { Cnano rrourn }=\mathbf{D} \overline{\mathbf{1}}
\end{aligned}
$$

\section{Crystal structure of compound 3}

Scheme S11.Synthesis of syn- $\boldsymbol{\beta}$-aminodisulfide 5.
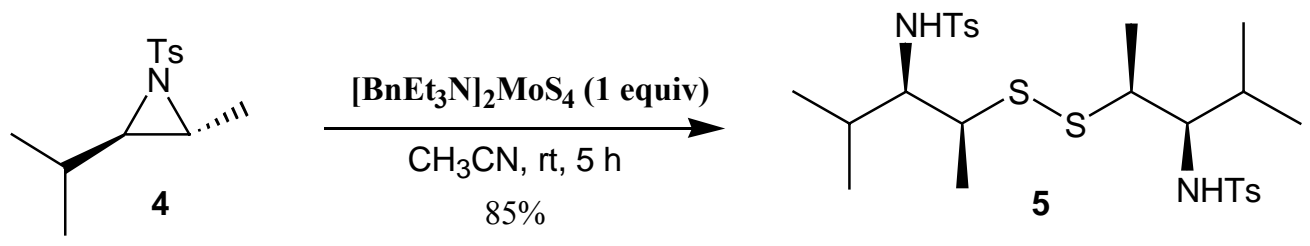

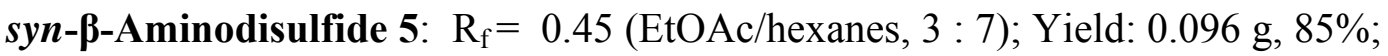

m. p: $142{ }^{\circ} \mathrm{C}$; IR (neat) $\mathrm{V}_{\max }: 3288,1512,1463,1323,1239,1207,1159,1905,828,754$, $668 \mathrm{~cm}^{-1} ;{ }^{1} \mathrm{H}$ NMR $\left(300 \mathrm{MHz}, \mathrm{CDCl}_{3}, 1: 1\right.$ mixture of diastereomers): $\delta 7.76(\mathrm{~d}$, $J=8.4 \mathrm{~Hz}, 4 \mathrm{H}), 7.28(\mathrm{~d}, J=8.4 \mathrm{~Hz}, 4 \mathrm{H}), 4.61(\mathrm{~d}, J=9.9 \mathrm{~Hz}, 1 \mathrm{H}), 4.57(\mathrm{~d}, J=9.6 \mathrm{~Hz}$, 1H), 3.37 (m, 2H), $2.89(\mathrm{~m}, 2 \mathrm{H}), 2.41(\mathrm{~s}, 6 \mathrm{H}), 1.97(\mathrm{~m}, 2 \mathrm{H}), 1.20(\mathrm{~d}, J=6.9 \mathrm{~Hz}, 6 \mathrm{H})$, $0.86(\mathrm{~d}, J=6.9 \mathrm{~Hz}, 6 \mathrm{H}), 0.77(\mathrm{~d}, J=5.7 \mathrm{~Hz}, 6 \mathrm{H}) ;{ }^{13} \mathrm{C} \mathrm{NMR}\left(75 \mathrm{MHz}, \mathrm{CDCl}_{3}, 1: 1\right.$ 
mixture of diastereomers): $\delta 143.1,138.6,129.4,127.1,127.0,62.4,62.3,50.5,50.4$, $30.0,29.9,21.5,20.6,20.5,17.9,17.8,17.7,17.6$; HR - MS m/z: calcd for $\mathrm{C}_{26} \mathrm{H}_{40} \mathrm{~N}_{2} \mathrm{O}_{4} \mathrm{~S}_{4} \mathrm{Na}^{+}\left[\mathrm{M}+\mathrm{Na}^{+}\right]$: 595.1769; found: 595.1780.

General procedure for the reaction of N-tosyl aziridino-tosylates with benzyltriethylammonium tetrathiomolybdate 1 . To a well-stirred solution of appropriate aziridine-tosylates $(0.50 \mathrm{mmol})$ in $\mathrm{CH}_{3} \mathrm{CN}(7 \mathrm{~mL})$ was added tetrathiomolybdate $1(0.616 \mathrm{~g}, 1.00 \mathrm{mmol})$ at once and stirred at room temperature $\left(28^{\circ} \mathrm{C}\right)$ for 3 to $6 \mathrm{~h}$. The solvent was evaporated under reduced pressure and the black residue was extracted with $\mathrm{CH}_{2} \mathrm{Cl}_{2}: \mathrm{Et}_{2} \mathrm{O}(1: 5,3 \times 10 \mathrm{~mL})$ and filtered through a celite pad. The filtrate was concentrated and the residue was purified by flash column chromatography on silica gel to give the appropriate sulfur heterocycles.

\section{Scheme S12.Synthesis of trans-thiirane 7 and trans-cyclic disulfide 8.}

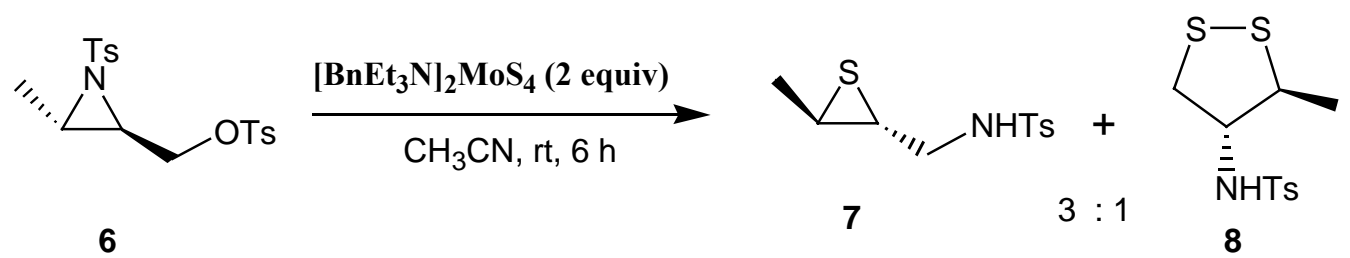

Trans-thiirane 7: $\mathrm{R}_{\mathrm{f}}=0.30$ (EtOAc/hexanes, $3: 7$ ); Yield: 0.048 g, 66\%; m. p: $92{ }^{\circ} \mathrm{C}$; IR (neat) $\mathrm{V}_{\max }: 3280,1597,1441,1324,1159,1093,1050,813,664 \mathrm{~cm}^{-1} ;{ }^{1} \mathrm{H}$ NMR (400 MHz, $\left.\mathrm{CDCl}_{3}\right): \delta$ 7.74, (d, $\left.J=8.4 \mathrm{~Hz}, 2 \mathrm{H}\right), 7.31(\mathrm{~d}, J=8.4 \mathrm{~Hz}, 2 \mathrm{H}), 4.91$ (t, $J=6.4 \mathrm{~Hz}, 1 \mathrm{H}), 3.27(\mathrm{~m}, 1 \mathrm{H}), 3.10(\mathrm{~m}, 1 \mathrm{H}), 2.74(\mathrm{~m}, 2 \mathrm{H}), 2.42(\mathrm{~s}, 3 \mathrm{H}), 1.44$ $(\mathrm{d}, J=5.6 \mathrm{~Hz}, 3 \mathrm{H}) ;{ }^{13} \mathrm{C} \mathrm{NMR}\left(75 \mathrm{MHz}, \mathrm{CDCl}_{3}\right): \delta 143.6,136.7,129.8,127.0,46.8,42.0$, 36.4, 21.4, 20.9; $\mathrm{HR}-\mathrm{MS} \mathrm{m} / \mathrm{z}$ : calcd for $\mathrm{C}_{11} \mathrm{H}_{15} \mathrm{NO}_{2} \mathrm{~S}_{2} \mathrm{Na}^{+}\left[\mathrm{M}+\mathrm{Na}^{+}\right]$: 280.0442; found: 280.0475 . 


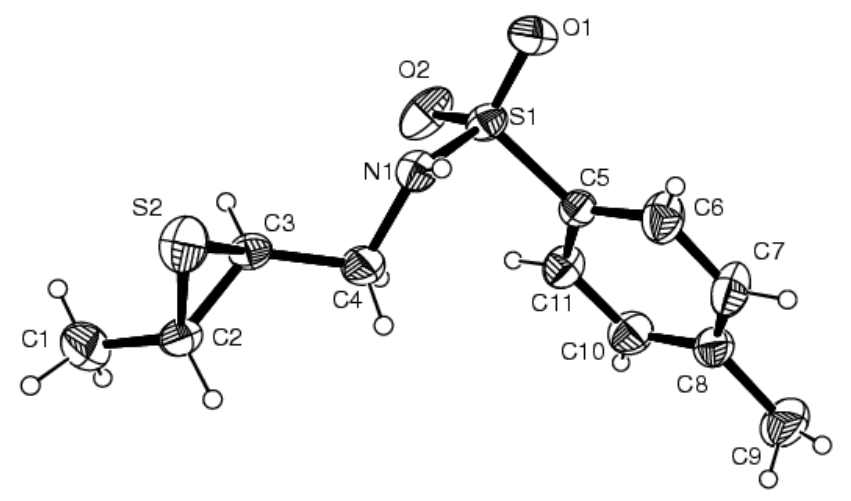

$$
\begin{aligned}
& \text { Crystal data of compound } 7 \\
& \begin{array}{ll}
\mathrm{a}=5.1696(30) & \alpha=90.000 \\
\mathrm{~b}=29.5060(12) & \beta=100.040(10) \\
\mathrm{c}=8.5218(50) & \gamma=90.000 \\
\mathrm{~V}=1279.96(26) & \\
\text { Space group }=\mathrm{P} 2_{1} / \mathrm{n}
\end{array}
\end{aligned}
$$

\section{Crystal structure of compound 7}

Cyclic disulfide 8: $\mathrm{R}_{\mathrm{f}}=0.40$ (EtOAc/hexanes, $3: 7$ ); Yield: $0.010 \mathrm{~g}, 16 \%$; m. p: $93{ }^{\circ} \mathrm{C}$; IR (neat) $\mathrm{V}_{\max }: 3274,2923,2853,1743,1333,1159,1091,813,666 \mathrm{~cm}^{-1}$; ${ }^{1} \mathrm{H}$ NMR (400 MHz, $\left.\mathrm{CDCl}_{3}\right): \delta 7.77(\mathrm{~d}, J=8.4 \mathrm{~Hz}, 2 \mathrm{H}), 7.33(\mathrm{~d}, J=8.4 \mathrm{~Hz}, 2 \mathrm{H}), 5.05$, (d, $J=10.4 \mathrm{~Hz}, 1 \mathrm{H}), 4.13(\mathrm{~m}, 1 \mathrm{H}), 3.40(\mathrm{~m}, 1 \mathrm{H}), 3.13(\mathrm{dd}, J=11.6,4.8 \mathrm{~Hz}, 1 \mathrm{H}), 2.89$ (dd, $J=11.6,2.0 \mathrm{~Hz}, 1 \mathrm{H}), 2.45(\mathrm{~s}, 3 \mathrm{H}), 1.24(\mathrm{~d}, J=6.8 \mathrm{~Hz}, 3 \mathrm{H}) ;{ }^{13} \mathrm{C} \mathrm{NMR}(75 \mathrm{MHz}$, $\left.\mathrm{CDCl}_{3}\right): \delta 143.9,137.8,129.9,126.9,64.7,55.4,42.2,21.5,20.17 ; \mathrm{HR}-\mathrm{MS} \mathrm{m} / \mathrm{z}$ : calcd for $\mathrm{C}_{11} \mathrm{H}_{15} \mathrm{NO}_{2} \mathrm{~S}_{3} \mathrm{Na}^{+}\left[\mathrm{M}+\mathrm{Na}^{+}\right]$: 312.0163 ; found: 312.0169 .

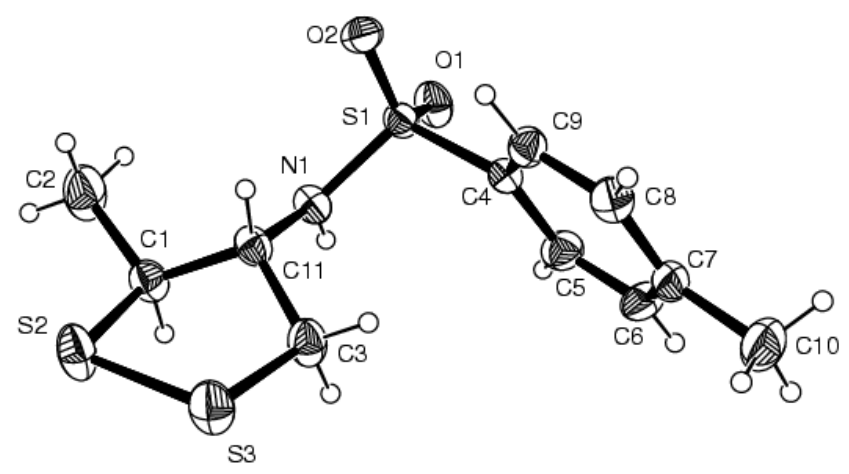

Crystal structure of compound 8

$$
\begin{aligned}
& \text { Crystal data of compound } 8 \\
& \begin{array}{ll}
\mathrm{a}=12.4697(73) & \alpha=90.000 \\
\mathrm{~b}=7.3474(43) & \beta=112.404(9) \\
\mathrm{c}=15.9649(93) & \gamma=90.000 \\
\mathrm{~V}=1352.30(56) & \\
\text { Space group }=\mathrm{P} 2_{1} / \mathrm{n}
\end{array}
\end{aligned}
$$

Scheme S13.Synthesis of tetrahydrothiophene derivative 10.<smiles>CC1(CC[OH2+])CC1</smiles>

9

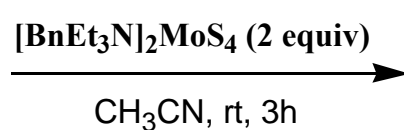

$88 \%$

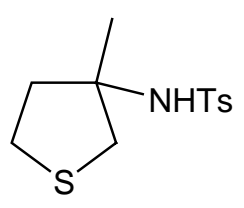

10 
Tetrahydrothiophene derivative 10: $\mathrm{R}_{\mathrm{f}}=0.70$ (EtOAc/hexanes, $3: 7$ ); Yield: 0.119 g, 88\%; m. p: $113{ }^{\circ} \mathrm{C}$; IR (neat) $\mathrm{V}_{\max }: 3237,1325,1152,1093,980,877,812$, $667 \mathrm{~cm}^{-1} ;{ }^{1} \mathrm{H}$ NMR $\left(300 \mathrm{MHz}, \mathrm{CDCl}_{3}\right): \delta 7.79(\mathrm{~d}, J=8.4 \mathrm{~Hz}, 2 \mathrm{H}), 7.29(\mathrm{~d}, J=8.4 \mathrm{~Hz}$, 2H), $5.14(\mathrm{~s}, 1 \mathrm{H}), 2.79(\mathrm{~m}, 2 \mathrm{H}), 2.77(\mathrm{dd}, J=11.4,1.5 \mathrm{~Hz}, 2 \mathrm{H}), 2.42(\mathrm{~s}, 3 \mathrm{H}), 2.33$ (m, 1H), $1.73(\mathrm{~m}, 1 \mathrm{H}), 1.43(\mathrm{~s}, 3 \mathrm{H}) ;{ }^{13} \mathrm{C}$ NMR $\left(75 \mathrm{MHz}, \mathrm{CDCl}_{3}\right): \delta 143.2,139.8,129.5$, 126.8, 66.2, 43.7, 41.8, 28.2, 23.3, 21.4; $\quad$ HR $\quad-\quad$ MS $\mathrm{m} / \mathrm{z}$ : calcd for $\mathrm{C}_{12} \mathrm{H}_{17} \mathrm{NO}_{2} \mathrm{~S}_{2} \mathrm{Na}^{+}\left[\mathrm{M}+\mathrm{Na}^{+}\right]: 294.0598$; found: 294.0627.

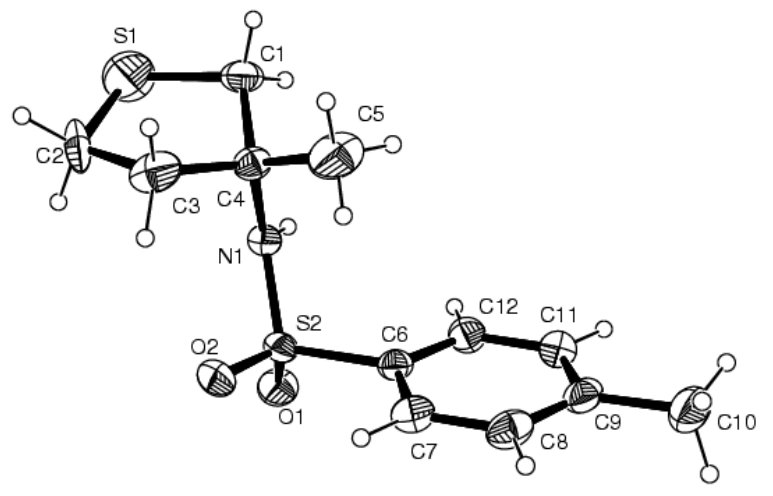

$$
\begin{aligned}
& \text { Crystal data of compound } \mathbf{1 0} \\
& \begin{array}{ll}
\mathrm{a}=10.0997(20) & \alpha=90.000 \\
\mathrm{~b}=6.3264(13) & \beta=94.225(4) \\
\mathrm{c}=20.7532(41) & \gamma=90.000 \\
\mathrm{~V}=1322.42(6) & \\
\text { Space group }=\mathrm{P} 2_{1} / \mathrm{n}
\end{array}
\end{aligned}
$$

\section{Crystal structure of compound 10}

Scheme S14. Synthesis of thia-bicyclic derivative 12.

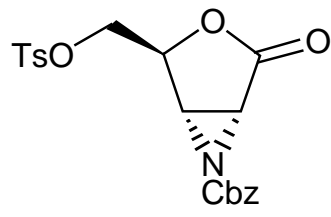

11

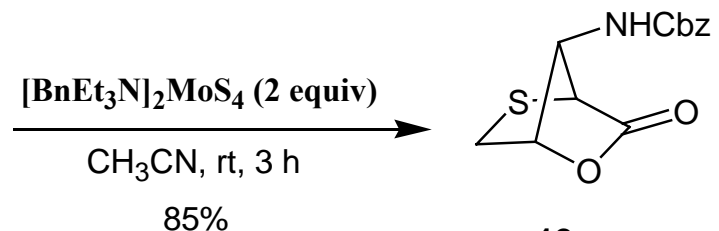

12

Thia-bicyclic derivative 12: $\mathrm{R}_{\mathrm{f}}=0.40($ EtOAc/hexanes, $3: 7)$; Yield: $0.118 \mathrm{~g}$, $85 \% ;[\alpha]_{D}^{27}=+60.00\left(\mathrm{c}=1.0, \mathrm{CHCl}_{3}\right) ;$ IR (neat) $\mathrm{V}_{\max }: 3305,1788,1703,1530,1264 \mathrm{~cm}^{-}$ ${ }^{1} ; \quad{ }^{1} \mathrm{H} \quad \mathrm{NMR} \quad\left(300 \mathrm{MHz}, \quad \mathrm{CDCl}_{3}\right): \quad \delta \quad 7.35 \quad(\mathrm{~m}, \quad 5 \mathrm{H}), \quad 5.40 \quad(\mathrm{bs}, \quad 1 \mathrm{H}), \quad 5.11$ (s, 2H), 4.99 (s, 1H), $4.20(\mathrm{~d}, J=6.3 \mathrm{~Hz}, 1 \mathrm{H}), 3.61(\mathrm{~s}, 1 \mathrm{H}), 3.28(\mathrm{dd}, J=11.5,1.8 \mathrm{~Hz}$, 
1H), $3.21(\mathrm{dd}, J=11.5,1.0 \mathrm{~Hz}, 1 \mathrm{H}) ;{ }^{13} \mathrm{C} \mathrm{NMR}\left(75 \mathrm{MHz}, \mathrm{CDCl}_{3}\right): \delta 171.8,155.6,135.5$, 128.6, 128.4, 128.2, 81.0, 67.5, 62.0, 44.2, 33.0; HR - MS m/z: calcd for $\mathrm{C}_{13} \mathrm{H}_{13} \mathrm{NO}_{4} \mathrm{SNa}^{+}\left[\mathrm{M}+\mathrm{Na}^{+}\right]: 302.0463$; found: 302.0474 .

Scheme S15. Synthesis of epoxy- $\beta$-amino sulfide 15.

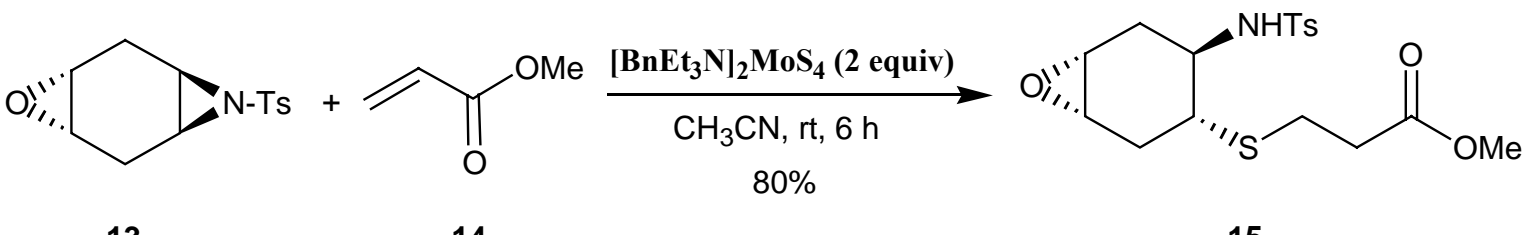

13 14

15

Epoxy- $\beta$-amino sulfide 15: Tetrathiomolybdate $1(0.609,1.00 \mathrm{mmol})$ was added to a well-stirred solution of trans-aziridino-epoxide $\mathbf{1 3}^{7}(0.132 \mathrm{~g}, 0.50 \mathrm{mmol})$ and methyl acrylate $14(0.055 \mathrm{~mL}, 0.60 \mathrm{mmol})$ in $\mathrm{CH}_{3} \mathrm{CN}(3 \mathrm{~mL})$ and the reaction mixture was stirred at room temperature $\left(28^{\circ} \mathrm{C}\right)$ for $6 \mathrm{~h}$. The solvent was evaporated under reduced pressure, and the black residue was extracted with $\mathrm{CH}_{2} \mathrm{Cl}_{2}: \mathrm{Et}_{2} \mathrm{O}(1: 5,3 \times 10 \mathrm{~mL})$ and filtered through a Celite pad. The filtrate was concentrated and the residue was purified by flash column chromatography on silica gel to give epoxy- $\beta$-aminodisulfide $\mathbf{1 5}$ as colorless oil. 15: $\mathrm{R}_{\mathrm{f}}=0.45$ (EtOAc/hexanes, $1: 1$ ); Yield: $0.540 \mathrm{~g}$, 71\%; IR (neat) $\mathrm{V}_{\max }$ : $3273,1735,1435,1328,1159,1091,815,669 \mathrm{~cm}^{-1} ;{ }^{1} \mathrm{H}$ NMR (300 MHz, $\left.\mathrm{CDCl}_{3}\right): \delta 7.77$ (d, $J=8.4 \mathrm{~Hz}, 2 \mathrm{H}), 7.31$ (d, $J=8.4 \mathrm{~Hz}, 2 \mathrm{H}), 5.27$ (d, $J=3.6 \mathrm{~Hz}, 1 \mathrm{H}), 3.71(\mathrm{~s}, 3 \mathrm{H}), 3.17$ (bs, $1 \mathrm{H}), 3.11(\mathrm{t}, J=4.2 \mathrm{~Hz}, 1 \mathrm{H}), 3.03(\mathrm{~m}, 1 \mathrm{H}), 2.87-2.81(\mathrm{~m}, 1 \mathrm{H}), 2.58-2.26(\mathrm{~m}, 6 \mathrm{H})$, $2.42(\mathrm{~s}, 3 \mathrm{H}), 2.12-2.02(\mathrm{~m}, 1 \mathrm{H}), 1.77(\mathrm{dq}, J=9.6,5.4,1.8 \mathrm{~Hz}, 1 \mathrm{H}) ;{ }^{13} \mathrm{C} \mathrm{NMR}(75 \mathrm{MHz}$, $\left.\mathrm{CDCl}_{3}\right): \delta 171.8,143.7,136.5,129.7,127.2,52.4,51.9,50.9,49.5,44.2,34.1,32.6,31.1$, 24.2, 21.5; HR - MS m/z: calcd for $\mathrm{C}_{17} \mathrm{H}_{23} \mathrm{NO}_{5} \mathrm{~S}_{2} \mathrm{Na}^{+}\left[\mathrm{M}+\mathrm{Na}^{+}\right]$: 408.0915; found: 408.0921. 


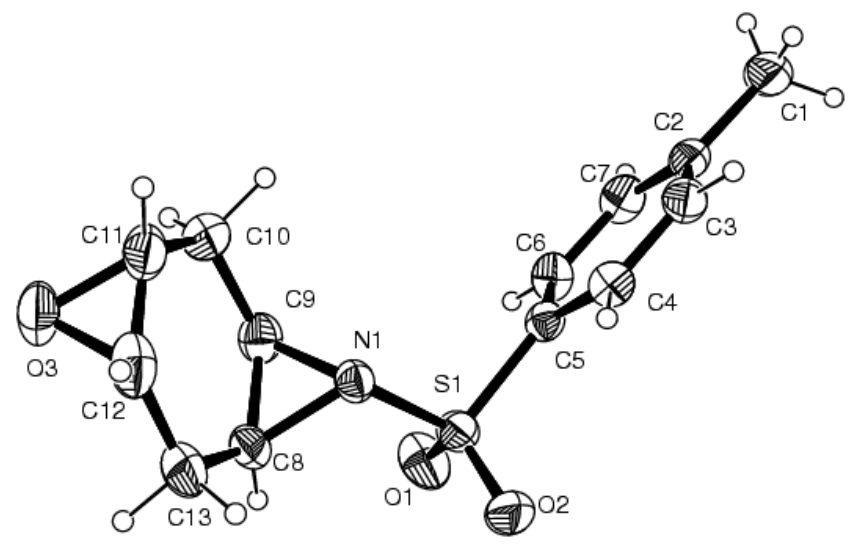

\section{Crystal data of compound 13}

$$
\begin{array}{ll}
\mathrm{a}=12.3840(20) & \alpha=90.000 \\
\mathrm{~b}=7.4375(12) & \beta=105.943(3) \\
\mathrm{c}=14.6124(24) & \gamma=90.000 \\
& \\
\mathrm{~V}=1294.12(24) & \\
\text { Space group }=\mathrm{P} 2_{1} / \mathrm{c} &
\end{array}
$$

\section{Crystal structure of compound 13}

Scheme S16. Synthesis of thia-bicyclononane derivatives 17 and 18.<smiles>CC1=CC[C@@H](C2CN2[AsH2])CC1=O</smiles>

16

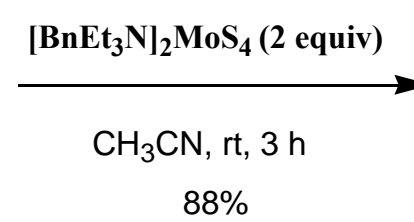

$88 \%$

Thia-bicyclononane derivatives 17 and 18: Tetrathiomolybdate 1 $(0.609,1.00 \mathrm{mmol})$ was added to a well-stirred solution of aziridine $\mathbf{1 6}(0.132 \mathrm{~g}, 0.50$ mmol) in $\mathrm{CH}_{3} \mathrm{CN}(3 \mathrm{~mL})$ and it was stirred at room temperature $\left(28{ }^{\circ} \mathrm{C}\right)$ for $3 \mathrm{~h}$. The solvent was evaporated under reduced pressure, the black residue was extracted with $\mathrm{CH}_{2} \mathrm{Cl}_{2}: \mathrm{Et}_{2} \mathrm{O}(1: 5,3 \times 10 \mathrm{~mL})$ and filtered through a Celite pad. The filtrate was concentrated and the residue was purified by flash column chromatography on silica gel to give thia-bicyclononane derivatives $\mathbf{1 7}$ and $\mathbf{1 8}$ as white solids.

17: $\mathrm{R}_{\mathrm{f}}=0.40$ (EtOAc/hexanes, 3 : 7); Yield: 0.048 g, 44\%; m. p: $174{ }^{\circ} \mathrm{C}$; $[\alpha]^{27}{ }_{D}=+97.00\left(\mathrm{c}=1.0, \mathrm{CHCl}_{3}\right) ; \mathrm{IR}$ (neat) $\mathrm{V}_{\max }: 3284,1706,1420,1316,1152,1018,667$ $\mathrm{cm}^{-1} ;{ }^{1} \mathrm{H}$ NMR (300 MHz, $\left.\mathrm{CDCl}_{3}\right): \delta 7.76(\mathrm{~d}, J=8.4 \mathrm{~Hz}, 2 \mathrm{H}), 7.28(\mathrm{~d}, J=8.4 \mathrm{~Hz}, 2 \mathrm{H})$, $5.82(\mathrm{~s}, 1 \mathrm{H}), 2.97(\mathrm{bs}, 1 \mathrm{H}), 2.86(\mathrm{bs}, 2 \mathrm{H}), 2.71(\mathrm{~m}, 1 \mathrm{H}), 2.58(\mathrm{~s}, 1 \mathrm{H}), 2.54(\mathrm{~d}, J=5.0 \mathrm{~Hz}$, 
2H), $2.41(\mathrm{~s}, 3 \mathrm{H}), 2.23(\mathrm{td}, J=7.2,3.3 \mathrm{~Hz}, 1 \mathrm{H}), 1.96(\mathrm{~d}, J=15.0 \mathrm{~Hz}, 1 \mathrm{H}), 1.24(\mathrm{~s}, 3 \mathrm{H})$, $1.23(\mathrm{~d}, J=6.0 \mathrm{~Hz}, 3 \mathrm{H}) ;{ }^{13} \mathrm{C} \mathrm{NMR}\left(75 \mathrm{MHz}, \mathrm{CDCl}_{3}\right): \delta 211.1,143.2,140.2,129.3,126.9$, 56.1, 49.6, 43.5, 41.8, 40.6, 33.6, 31.6, 23.8, 21.4, 12.8; HR - MS m/z: calcd for $\mathrm{C}_{13} \mathrm{H}_{19} \mathrm{NO}_{2} \mathrm{SNa}^{+}\left[\mathrm{M}+\mathrm{Na}^{+}\right]: 376.1017$; found: 376.1033 .

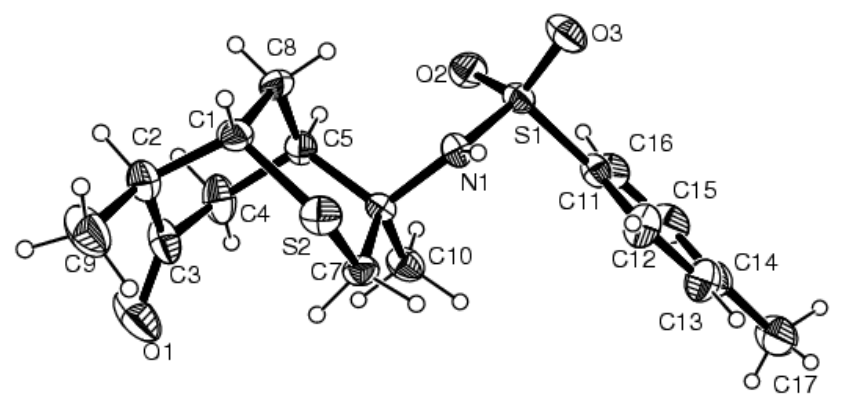

Crystal structure of compound 17

\begin{tabular}{|ll} 
Crystal data of compound $\mathbf{1 7}$ \\
$\mathrm{a}=10.8791(75)$ & $\alpha=90.000$ \\
$\mathrm{~b}=6.2139(43)$ & $\beta=106.637910)$ \\
$\mathrm{c}=13.8138(96)$ & $\gamma=90.000$ \\
& \\
$\mathrm{~V}=894.74(30)$ & \\
Space group $=\mathrm{P} 2_{1}$ &
\end{tabular}

18: $\mathrm{R}_{\mathrm{f}}=0.30\left(\right.$ EtOAc/hexanes, 3 : 7); Yield: 0.48 g, 44\%; m. p: $223{ }^{\circ} \mathrm{C}$; $[\alpha]_{D}^{27}=+137.00\left(\mathrm{c}=1.0, \mathrm{CHCl}_{3}\right) ; \mathrm{IR}$ (neat) $\mathrm{V}_{\max }: 3274,1704,1332,1159,1091,909$, 815, 732, $663 \mathrm{~cm}^{-1} ;{ }^{1} \mathrm{H}$ NMR $\left(300 \mathrm{MHz}, \mathrm{CDCl}_{3}\right): \delta 7.74(\mathrm{~d}, J=8.4 \mathrm{~Hz}, 2 \mathrm{H}), 7.29(\mathrm{~d}$, $J=8.4 \mathrm{~Hz}, 2 \mathrm{H}), 4.74(\mathrm{~s}, 1 \mathrm{H}), 2.92(\mathrm{~m}, 2 \mathrm{H}), 2.71-2.45(\mathrm{~m}, 5 \mathrm{H}), 2.42(\mathrm{~s}, 3 \mathrm{H}), 2.31(\mathrm{td}$, $J=13.8,3.6 \mathrm{~Hz}, 1 \mathrm{H}), 2.05(\mathrm{~d}, J=13.8 \mathrm{~Hz}, 1 \mathrm{H}), 1.64(\mathrm{~s}, 3 \mathrm{H}), 1.19(\mathrm{~d}, \mathrm{~J}=6.6 \mathrm{~Hz}, 3 \mathrm{H})$; ${ }^{13} \mathrm{C}$ NMR $\left(75 \mathrm{MHz}, \mathrm{CDCl}_{3}\right): \delta 211.1,143.3,140.1,129.6,126.8,58.9,50.0,42.6,41.9$, $41.4, \quad 32.9, \quad 31.5, \quad 22.9, \quad 21.5, \quad 12.6 ; \quad \mathrm{HR} \quad-\quad \mathrm{MS} \quad \mathrm{m} / \mathrm{z}$ : calcd for $\mathrm{C}_{13} \mathrm{H}_{19} \mathrm{NO}_{2} \mathrm{SNa}^{+}\left[\mathrm{M}+\mathrm{Na}^{+}\right]: 376.1017$; found: 376.1030 .

General procedure for the reaction of aziridino-epoxide with benzyltriethylammonium tetrathiomolybdate 1: To a well-stirred solution of appropriate aziridino-epoxide $(0.50 \mathrm{mmol})$ in $\mathrm{CH}_{3} \mathrm{CN}: \mathrm{EtOH}(1: 1,10 \mathrm{~mL})$ was added tetrathiomolybdate $1(0.609 \mathrm{~g}, 1.00 \mathrm{mmol})$ at once and stirred at room temperature $\left(28{ }^{\circ} \mathrm{C}\right)$ for $7-12 \mathrm{~h}$. The solvent was evaporated under reduced pressure and the black 
residue was extracted with $\mathrm{CH}_{2} \mathrm{Cl}_{2}: \mathrm{Et}_{2} \mathrm{O}(1: 5,3 \times 10 \mathrm{~mL})$ and filtered through a Celite pad. The filtrate was concentrated and residue was purified by flash column chromatography on silica gel to give the desired product in excellent yield.

Scheme S17.Synthesis of bridged bicyclic disulfide 20.
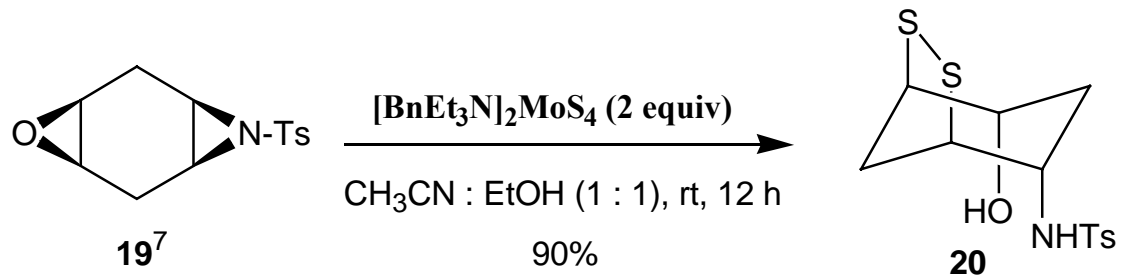

Bridged bicyclic disulfide 20: $\mathrm{R}_{\mathrm{f}}=0.50$ (EtOAc/hexanes, $\left.1: 1\right)$; Yield: $0.149 \mathrm{~g}$, 90\%; m. p: $155^{\circ} \mathrm{C}$; IR (neat) $\mathrm{V}_{\max }: 3480,3316,1416,1326,1156,1084,1024,667 \mathrm{~cm}^{-1}$; ${ }^{1} \mathrm{H}$ NMR $\left(300 \mathrm{MHz}, \mathrm{CDCl}_{3}\right): \delta 7.73(\mathrm{~d}, J=8.4 \mathrm{~Hz}, 2 \mathrm{H}), 7.31(\mathrm{~d}, J=8.4 \mathrm{~Hz}, 2 \mathrm{H}), 6.03$ (d, $J=8.4 \mathrm{~Hz}, 1 \mathrm{H}), 3.99(\mathrm{t}, J=3.9 \mathrm{~Hz}, 1 \mathrm{H}), 3.92(\mathrm{bs}, 1 \mathrm{H}), 3.81(\mathrm{t}, J=3.9 \mathrm{~Hz}, 1 \mathrm{H}), 3.48$ (bs, 1H), 3.43 (bs, 1H), 2.48 (t, $J=3.9 \mathrm{~Hz}, 1 \mathrm{H}), 2.43$ (s, 3H), 2.37 (d, $J=1.8 \mathrm{~Hz}, 1 \mathrm{H})$, $1.87(\mathrm{td}, J=13.2,4.2 \mathrm{~Hz}, 1 \mathrm{H}), 1.26(\mathrm{bd}, J=13.2 \mathrm{~Hz}, 1 \mathrm{H}) ;{ }^{13} \mathrm{C} \mathrm{NMR}\left(75 \mathrm{MHz}, \mathrm{CDCl}_{3}\right): \delta$ 143.5, 137.5, 129.8, 126.8, 71.0, 54.1, 52.2, 52.1, 34.5, 28.4, 21.5; HR - MS m/z: calcd for $\mathrm{C}_{13} \mathrm{H}_{17} \mathrm{NO}_{3} \mathrm{~S}_{3} \mathrm{Na}^{+}\left[\mathrm{M}+\mathrm{Na}^{+}\right]$: 354.0268; found: 354.0273 .

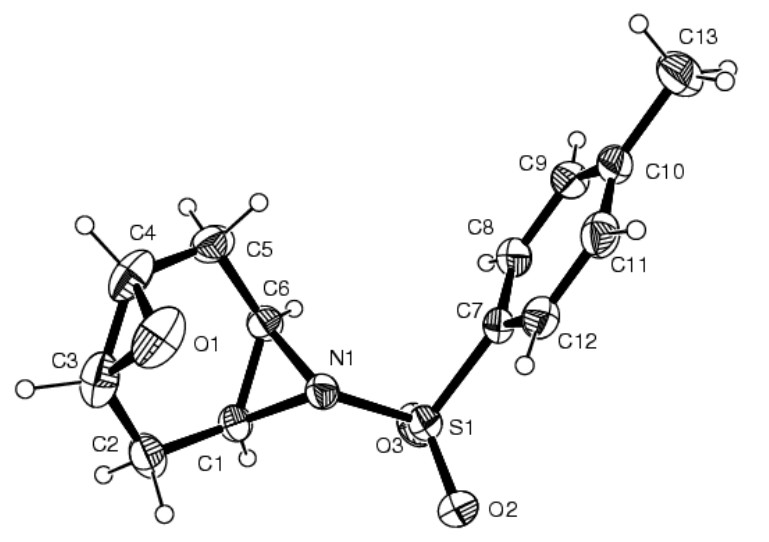

$$
\begin{aligned}
& \text { Crystal data of compound } 19 \\
& \mathrm{a}=6.3004(12) \quad \alpha=90.000 \\
& \mathrm{~b}=25.6737(49) \quad \beta=91.402(3) \\
& \mathrm{c}=15.7821(30) \quad \gamma=90.000 \\
& \mathrm{~V}=2552.06(9) \\
& \text { Space group }=\mathrm{P} 2{ }_{1} / \mathrm{n}
\end{aligned}
$$

\section{Crystal structure of compound 19}




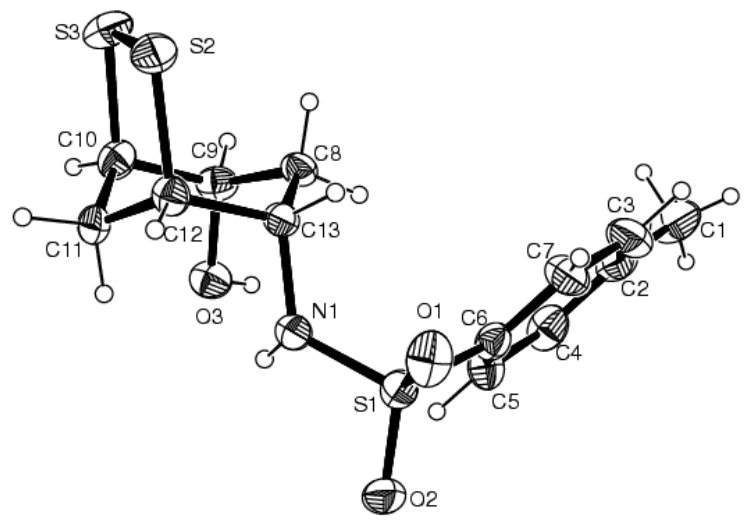

$$
\begin{aligned}
& \text { Crystal data of compound } 20 \\
& \begin{array}{ll}
\mathrm{a}=6.6883(45) & \alpha=99.613(9) \\
\mathrm{b}=10.7402(71) & \beta=99.698(9) \\
\mathrm{c}=12.0919(80) & \gamma=97.747(9) \\
\mathrm{V}=832.22(27) & \\
\text { Space group }=\mathrm{P} \overline{\mathbf{1}} &
\end{array}
\end{aligned}
$$

\section{Crystal structure of compound 20}

Scheme S18.Synthesis of thia-bicyclononane derivative 22.

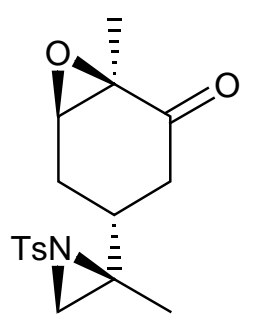

21

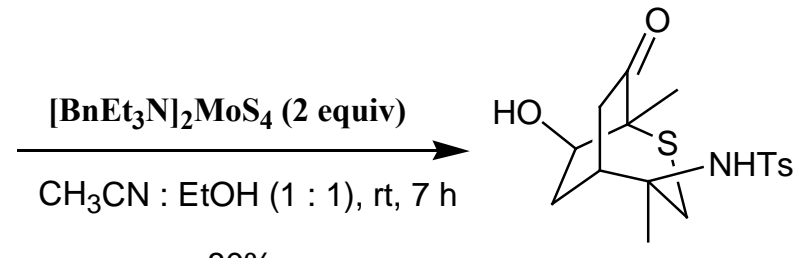

$80 \%$

22

Thia-bicyclononane derivative 22: $R_{\mathrm{f}}=0.45$ (EtOAc/hexanes, $3: 7$ ); Yield: $0.147 \mathrm{~g}, 80 \%$; m. p: $152{ }^{\circ} \mathrm{C} ;[\alpha]^{27}{ }_{\mathrm{D}}=+47.00\left(\mathrm{c}=1.0, \mathrm{CHCl}_{3}\right) ; \mathrm{IR}$ (neat) $\mathrm{V}_{\max }: 3498,3278$, 1707, 1319, 1154, 1090, 1043, 972, 815, 736, $664 \mathrm{~cm}^{-1} ;{ }^{1} \mathrm{H}$ NMR (300 MHz, $\left.\mathrm{CDCl}_{3}\right): \delta$ $7.73(\mathrm{~d}, J=8.5 \mathrm{~Hz}, 2 \mathrm{H}), 7.29(\mathrm{~d}, J=8.5 \mathrm{~Hz}, 2 \mathrm{H}), 4.88(\mathrm{~s}, 1 \mathrm{H}), 4.17(\mathrm{dd}, J=7.8,2.4 \mathrm{~Hz}$, 1H), $2.84(\mathrm{dd}, J=17.4,2.4 \mathrm{~Hz}, 1 \mathrm{H}), 2.73(\mathrm{bd}, J=15.0 \mathrm{~Hz}, 1 \mathrm{H}), 2.59$ (bs, 1H), 2.53 (t, $J=5.1 \mathrm{~Hz}, 2 \mathrm{H}), 2.44(\mathrm{~s}, 1 \mathrm{H}), 2.42(\mathrm{~s}, 3 \mathrm{H}), 1.88(\mathrm{dq}, J=8.2,2.1 \mathrm{~Hz}, 1 \mathrm{H}), 1.57$ (bs, 1H), $1.43(\mathrm{~s}, 3 \mathrm{H}), 1.36(\mathrm{~s}, 3 \mathrm{H}) ;{ }^{13} \mathrm{C} \mathrm{NMR}\left(75 \mathrm{MHz}, \mathrm{CDCl}_{3}\right): \delta$ 205.2, 143.4, 139.9, 129.7, $126.8,74.3,61.6,52.8,39.6,38.9,38.3,32.2,24.0,21.5,19.4 ;$ HR - MS m/z: calcd for $\mathrm{C}_{17} \mathrm{H}_{23} \mathrm{NO}_{4} \mathrm{~S}_{2} \mathrm{Na}^{+}\left[\mathrm{M}+\mathrm{Na}^{+}\right]$: 392.0966; found: 392.0997 . 


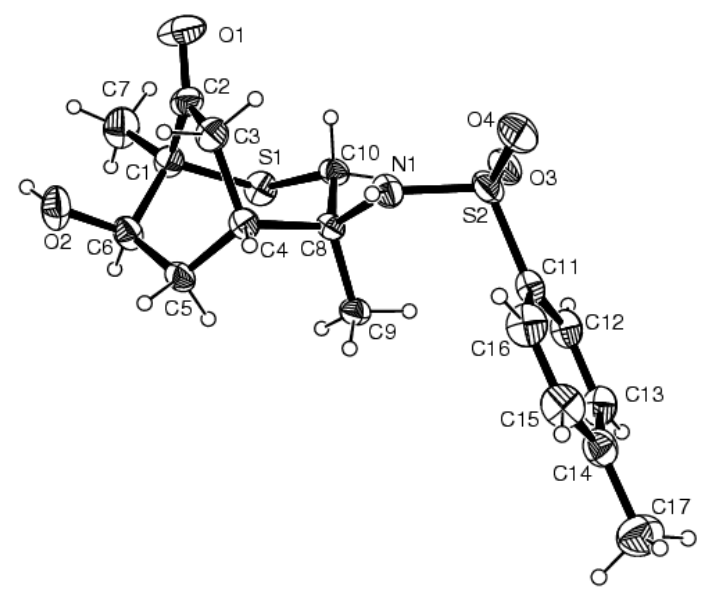

$$
\begin{aligned}
& \text { Crystal data of compound } 22 \\
& \begin{array}{ll}
\mathrm{a}=8.0979(44) & \alpha=90.000 \\
\mathrm{~b}=10.3725(57) & \beta=90.000 \\
\mathrm{c}=21.5805(12) & \gamma=90.000 \\
\mathrm{~V}=1812.669(17) & \\
\text { Space group }=\mathrm{P} 2{ }_{1}{ }_{1}{ }_{1}{ }_{1}
\end{array}
\end{aligned}
$$

\section{Crystal structure of compound 22}

\section{General procedure for the reaction of bis-aziridines with}

tetrathiomolybdate 1: To a well-stirred solution of appropriate bis-aziridine ( 0.50 mmol) in $\mathrm{CH}_{3} \mathrm{CN}(8 \mathrm{~mL})$ was added tetrathiomolybdate $1(0.609 \mathrm{~g}, 1 \mathrm{mmol})$ at once and stirred at room temperature $\left(28^{\circ} \mathrm{C}\right)$ for $1-4 \mathrm{~h}$. The solvent was evaporated under reduced pressure and the black residue was extracted with $\mathrm{CH}_{2} \mathrm{Cl}_{2}: \mathrm{Et}_{2} \mathrm{O}(1: 5,3 \times 10 \mathrm{~mL})$ and filtered through a Celite pad. The filtrate was concentrated and the residue was purified by flash column chromatography on silica gel to give the desired product in excellent yield.

\section{Scheme S19.Synthesis of cyclic sulfide 24 and disulfide 25.}

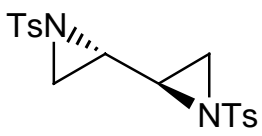

$23^{8}$

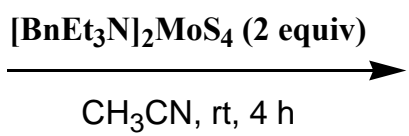

$\mathrm{CH}_{3} \mathrm{CN}, \mathrm{rt}, 4 \mathrm{~h}$

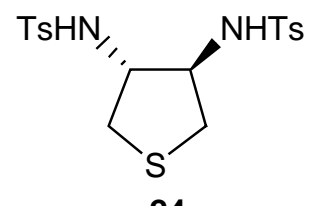

24

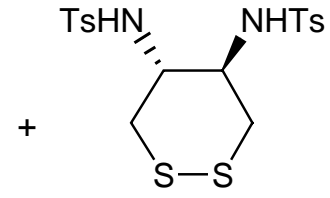

$1: 1$
25

Cyclic sulfide 24: $\mathrm{R}_{\mathrm{f}}=0.40$ (EtOAc/hexanes, 3 : 7); Yield: 0.090 g, 42\%; m. p: $153{ }^{\circ} \mathrm{C} ; ;[\alpha]^{27}{ }_{\mathrm{D}}=-53.00\left(\mathrm{c}=1.0, \mathrm{CHCl}_{3}\right) ; \mathrm{IR}$ (neat) $\mathrm{V}_{\max }: 3269,1597,1454,1093,1330$, 
1159, 1092, 814, $666 \mathrm{~cm}^{-1} ;{ }^{1} \mathrm{H}$ NMR $\left(300 \mathrm{MHz}, \mathrm{CDCl}_{3}\right): \delta 7.76(\mathrm{~d}, J=8.4 \mathrm{~Hz}, 2 \mathrm{H}), 7.34$

(d, $J=8.4 \mathrm{~Hz}, 2 \mathrm{H}), 4.94(\mathrm{~d}, J=6.0 \mathrm{~Hz}, 1 \mathrm{H}), 3.73(\mathrm{~m}, 1 \mathrm{H}), 2.93(\mathrm{dd}, J=11.4,5.1 \mathrm{~Hz}$,

$1 \mathrm{H}), 2.46(\mathrm{dd}, J=16.5,5.1 \mathrm{~Hz}, 1 \mathrm{H}), 2.45(\mathrm{~s}, 3 \mathrm{H}) ;{ }^{13} \mathrm{C} \mathrm{NMR}\left(75 \mathrm{MHz}, \mathrm{CDCl}_{3}\right): \delta 144.0$,

136.4, 129.9, 127.2, 60.0, 32.6, 21.5; $\quad$ HR $\quad-\quad$ MS $\mathrm{m} / \mathrm{z}: \quad$ calcd for $\mathrm{C}_{18} \mathrm{H}_{22} \mathrm{~N}_{2} \mathrm{O}_{4} \mathrm{~S}_{3} \mathrm{Na}^{+}\left[\mathrm{M}+\mathrm{Na}^{+}\right]:$449.0693; found: 449.0655 .

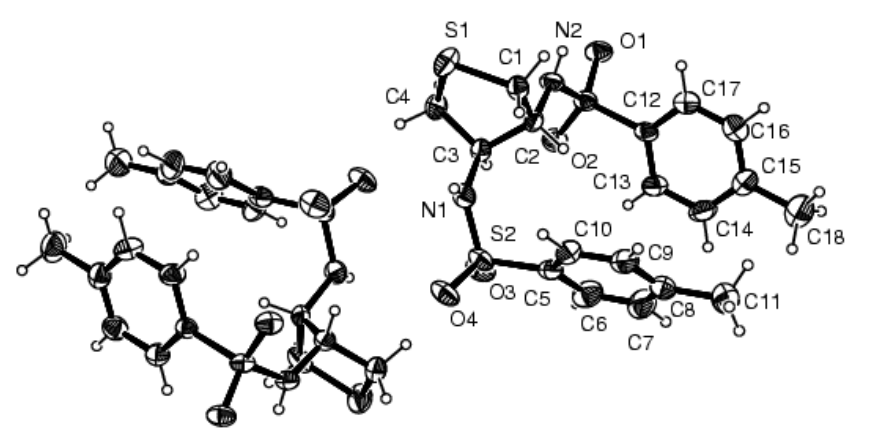

Crystal structure of compound 24

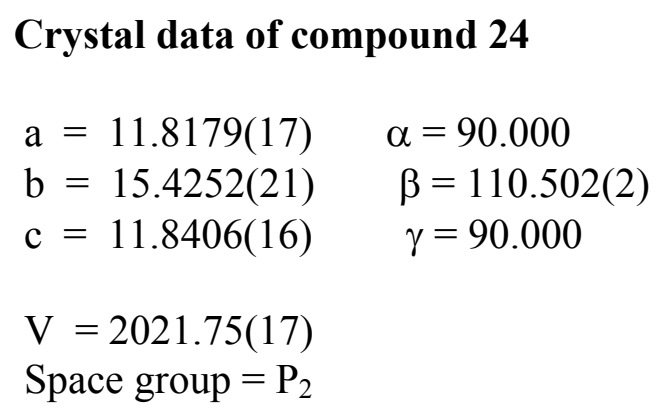

Cyclic disulfide 25: $\mathrm{R}_{\mathrm{f}}=0.50$ (EtOAc/hexanes, $3: 7$ ); Yield: $0.100 \mathrm{~g}, 43 \%$; m. p: $204{ }^{\circ} \mathrm{C} ;[\alpha]^{27}=-62.00\left(\mathrm{c}=1.0, \mathrm{CHCl}_{3}\right)$; IR (neat) $\mathrm{V}_{\max }: 3267,1597,1454,1330$, 1159, $1092814,666 \mathrm{~cm}^{-1} ;{ }^{1} \mathrm{H}$ NMR $\left(300 \mathrm{MHz}, \mathrm{CDCl}_{3}: \mathrm{DMSO}_{6}\right): \delta 7.73$ (d, $J=8.5 \mathrm{~Hz}, 2 \mathrm{H}), 7.30$ (d, $J=7.5 \mathrm{~Hz}, 2 \mathrm{H}), 6.72$ (bs, 1H), 3.44 (bs, 1H), 3.09 (bs, 1H), 2.85 (bs, 1H), $2.43(\mathrm{~s}, 3 \mathrm{H}) ;{ }^{13} \mathrm{C}$ NMR $\left(75 \mathrm{MHz}, \mathrm{CDCl}_{3}\right): \delta 143.2,136.9,129.5,126.9$, 21.35; HR - MS m/z: calcd for $\mathrm{C}_{18} \mathrm{H}_{22} \mathrm{~N}_{2} \mathrm{O}_{4} \mathrm{~S}_{4} \mathrm{Na}^{+}\left[\mathrm{M}+\mathrm{Na}^{+}\right]$: 481.0360 ; found: 481.0369 .

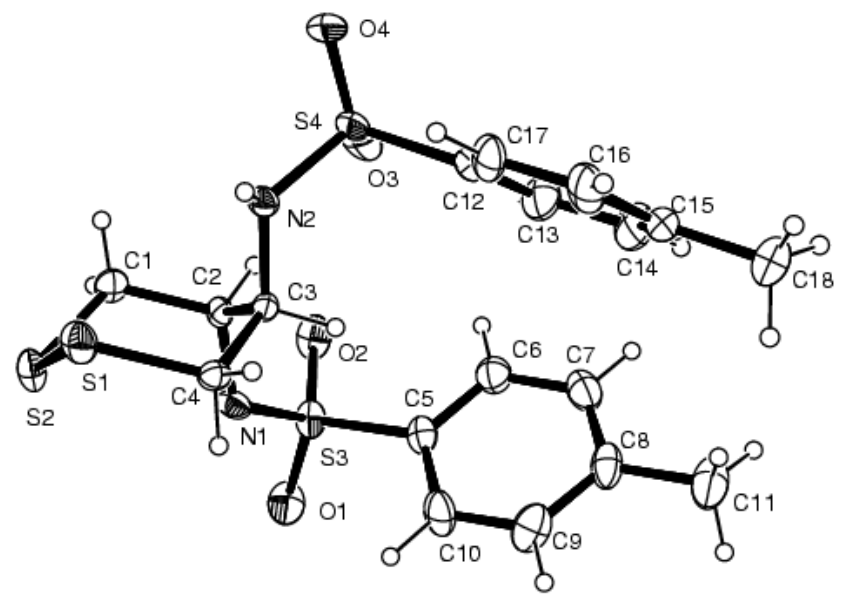

$$
\begin{aligned}
& \text { Crystal data of compound } 25 \\
& \begin{array}{ll}
\mathrm{a}=6.4480(8) & \alpha=90.000 \\
\mathrm{~b}=14.3862(2) & \beta=90.000 \\
\mathrm{c}=22.7353(3) & \gamma=90.000 \\
\mathrm{~V}=2108.98(5) & \\
\text { Space group }=\mathrm{P} 22_{1} 2_{1} 2_{1}
\end{array}
\end{aligned}
$$


Scheme S20.Synthesis of thiepane derivative 27

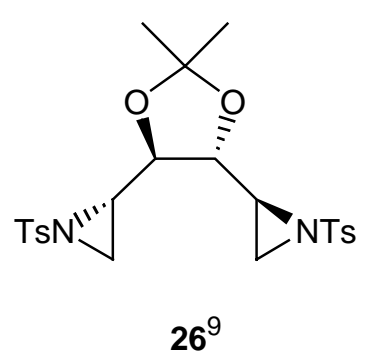

$26^{9}$

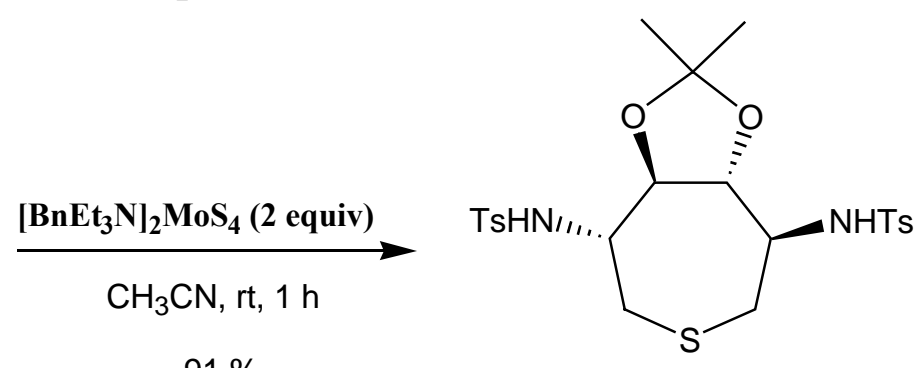

27

Thiepane 27: $\mathrm{R}_{\mathrm{f}}=0.45$ (EtOAc/hexanes, 3 : 7); Yield: $0.237 \mathrm{~g}, 91 \%$; m. p: $251{ }^{\circ} \mathrm{C} ;[\alpha]^{27}{ }_{\mathrm{D}}=+34.00\left(\mathrm{c}=1.0, \mathrm{CHCl}_{3}\right)$; IR (neat) $\mathrm{V}_{\max }: 3271,1443,1329,1149$, 1088, 1076, 812, $664 \mathrm{~cm}^{-1} ;{ }^{1} \mathrm{H}$ NMR $\left(300 \mathrm{MHz}, \mathrm{CDCl}_{3}:\right.$ DMSO-d 6$): \delta 7.71$ (d, $J=8.3 \mathrm{~Hz}, 4 \mathrm{H}), 7.24(\mathrm{~d}, J=8.3 \mathrm{~Hz}, 4 \mathrm{H}), 6.72(\mathrm{~d}, J=6.3 \mathrm{~Hz}, 2 \mathrm{H}), 3.80(\mathrm{~m}, 2 \mathrm{H}), 3.33$ (m, 2H), $2.84(\mathrm{dd}, J=15.3,4.8 \mathrm{~Hz}, 2 \mathrm{H}), 2.71(\mathrm{dd}, J=15.3,6.3 \mathrm{~Hz}, 2 \mathrm{H}), 2.39(\mathrm{~s}, 6 \mathrm{H})$, $0.87(\mathrm{~s}, 6 \mathrm{H}) ;{ }^{13} \mathrm{C} \mathrm{NMR}\left(75 \mathrm{MHz}, \mathrm{CDCl}_{3}\right): \delta 142.5,137.4,128.7,126.8,108.3,56.8,37.9$, 25.6, 21.0; HR - MS m/z: calcd for $\mathrm{C}_{23} \mathrm{H}_{30} \mathrm{~N}_{2} \mathrm{O}_{6} \mathrm{~S}_{3} \mathrm{Na}^{+}\left[\mathrm{M}+\mathrm{Na}^{+}\right]$: 549.1164; found: 549.1173.
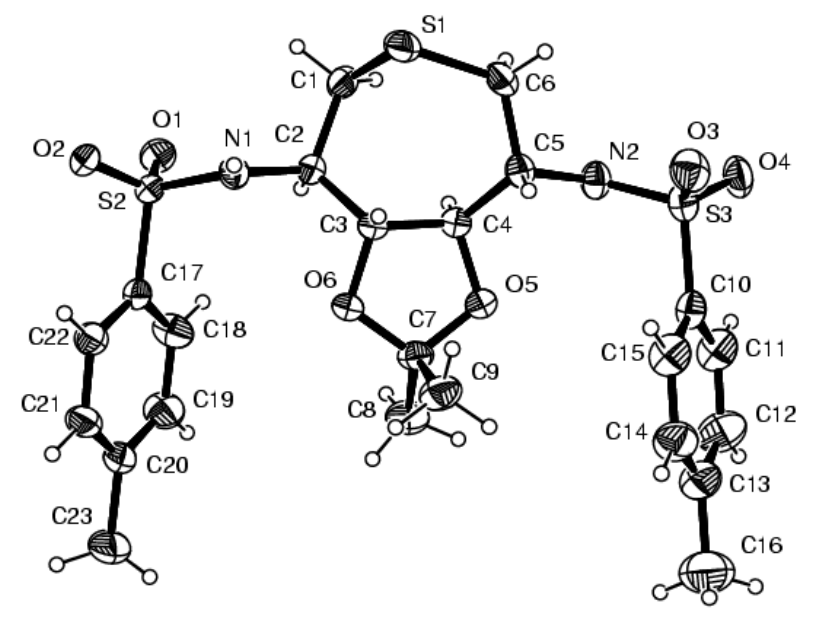

\begin{tabular}{|c|c|}
\hline \multicolumn{2}{|c|}{ Crystal data of compound 27} \\
\hline $\mathrm{a}=9.9407(57)$ & $\alpha=90.000$ \\
\hline $\mathrm{b}=9.1610(53)$ & $\beta=109.232(9)$ \\
\hline $\mathrm{c}=15.0456(89)$ & $\gamma=90.000$ \\
\hline $\mathrm{V}=1293.69(45)$ & \\
\hline Space group $=\mathrm{P} 2$ & \\
\hline
\end{tabular}

\section{Crystal structure of compound 27}

Refinement strategy: Two carbon atoms C8 and C9 have high values of anisotropic displacement parameter. These carbons were modeled and this was split up and the occupancies refined to a final value of $0.64: 0.36$ for $\mathrm{C} 8$ and $0.6: 0.4$ for $\mathrm{C} 9$. 


\section{References}

1. Kruss, G. Justus Liebigs Ann.Chem. 1884, 225, 1.

2. Ramesha, A. R.; Chandrasekaran, S. Synth. Commun. 1992, 22, 3277 - 3284.

3. Jeong, J. U.; Tao, B.; Sagasser, I.; Henniges, H.; Sharpless, K. B. J. Am. Chem. Soc. 1998, 120, 6844 - 6845

4. Klein, E.; Ohloff, G. Tetrahedron 1963, 19, 1091 - 1099.

5. Pearson, W. H.; Hines, J. V. J. Org. Chem. 2000, 65, 5785 - 5793.

6. Tarrade, A.; Dauban.; Dodd, R. H. J. Org. Chem. 2003, 68, 9521 - 9524.

7. Brien, O. P.; Pilgram, C. D. Org. Biomol. Chem. 2003, 1, 523 - 534.

8. Kanger, T.; Ausmees, K.; Muurisepp, A. M.; Pehk, T.; Loop, M. Synlett. 2003, $1055-1057$.

9. Merrer, Y. L.; Dureult, A.; Greck, C.; Langum, D. M.; Graier, C.; Depezay, J. Heterocycles, 1987, 25, 541 - 548. 

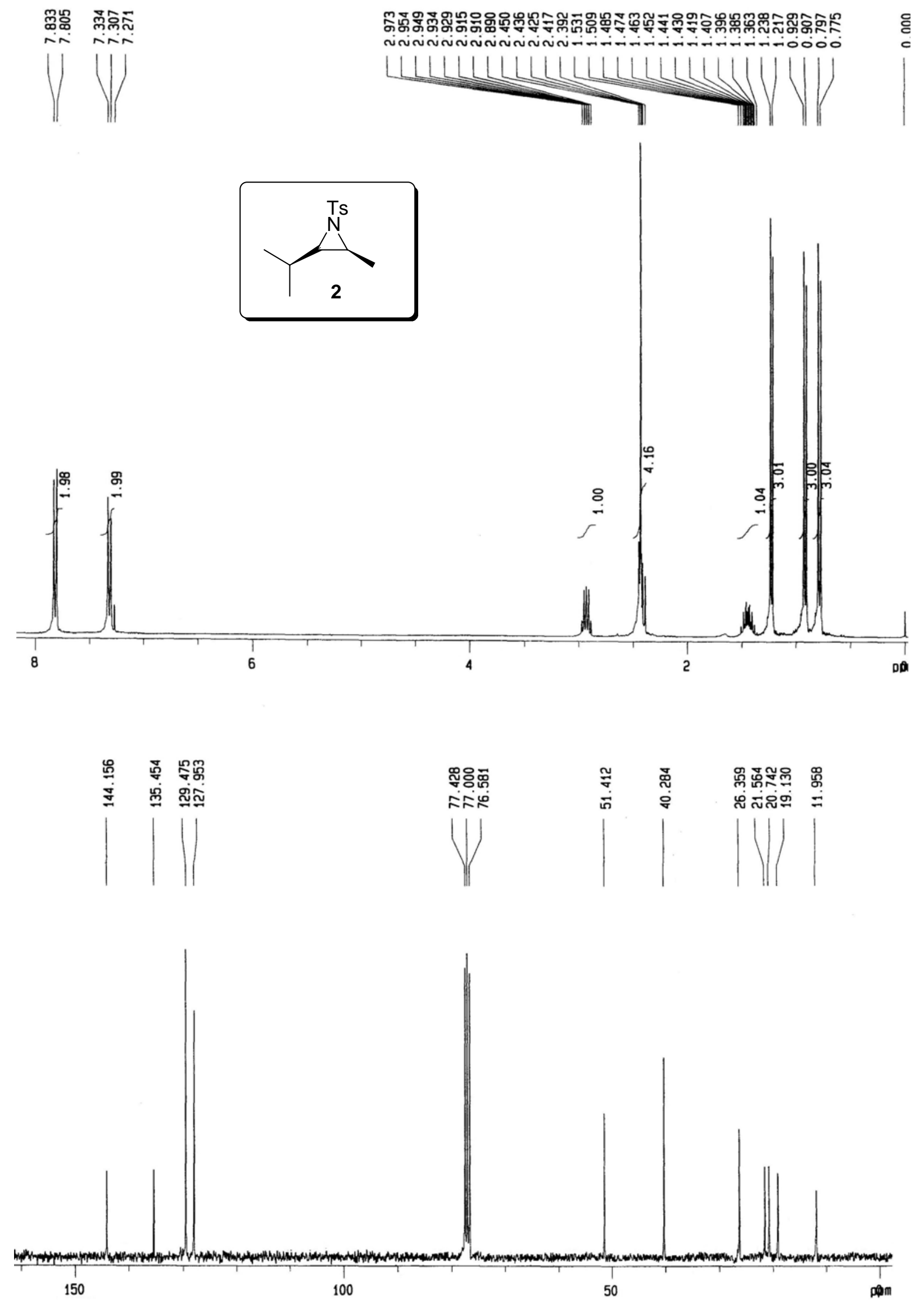

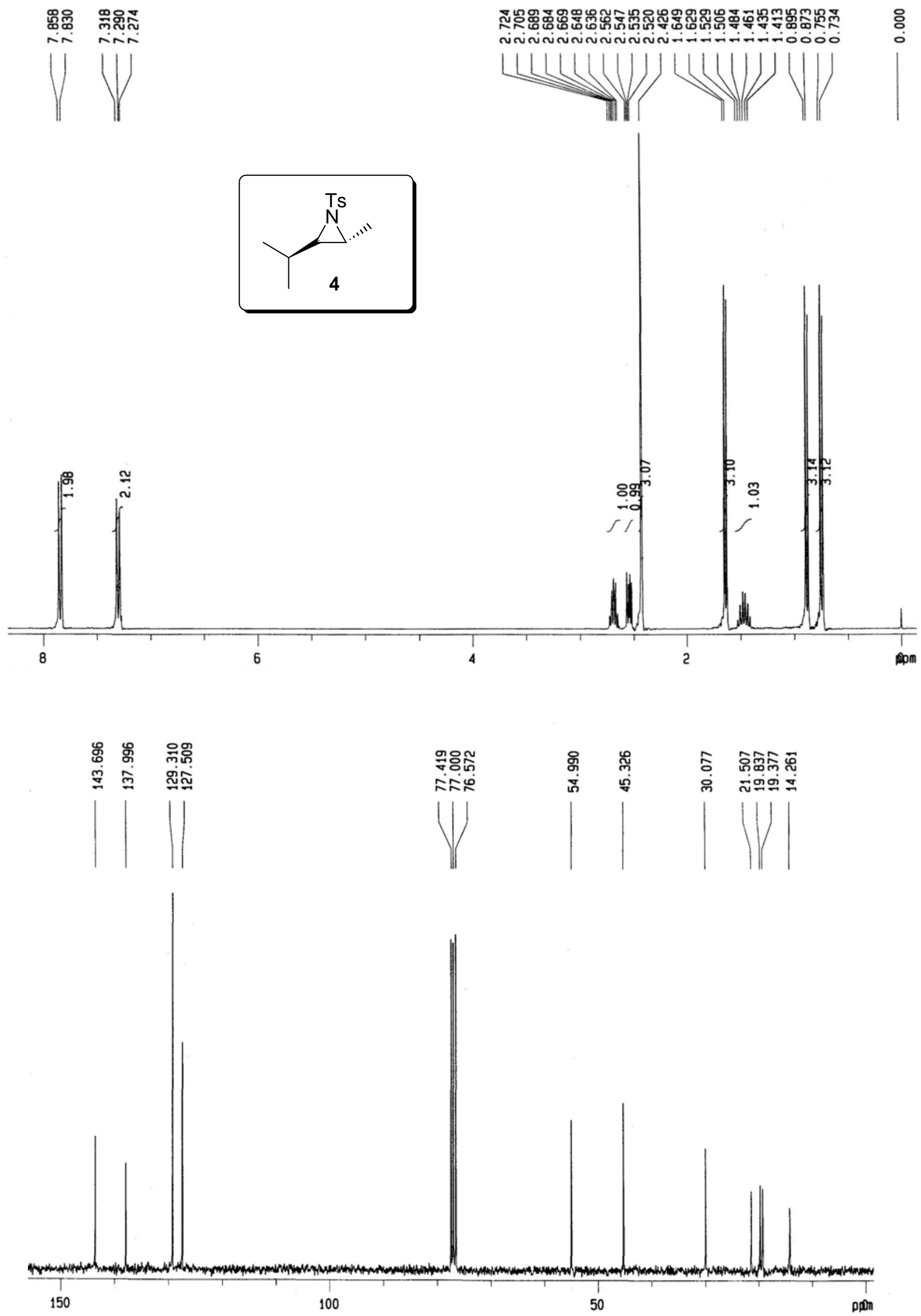


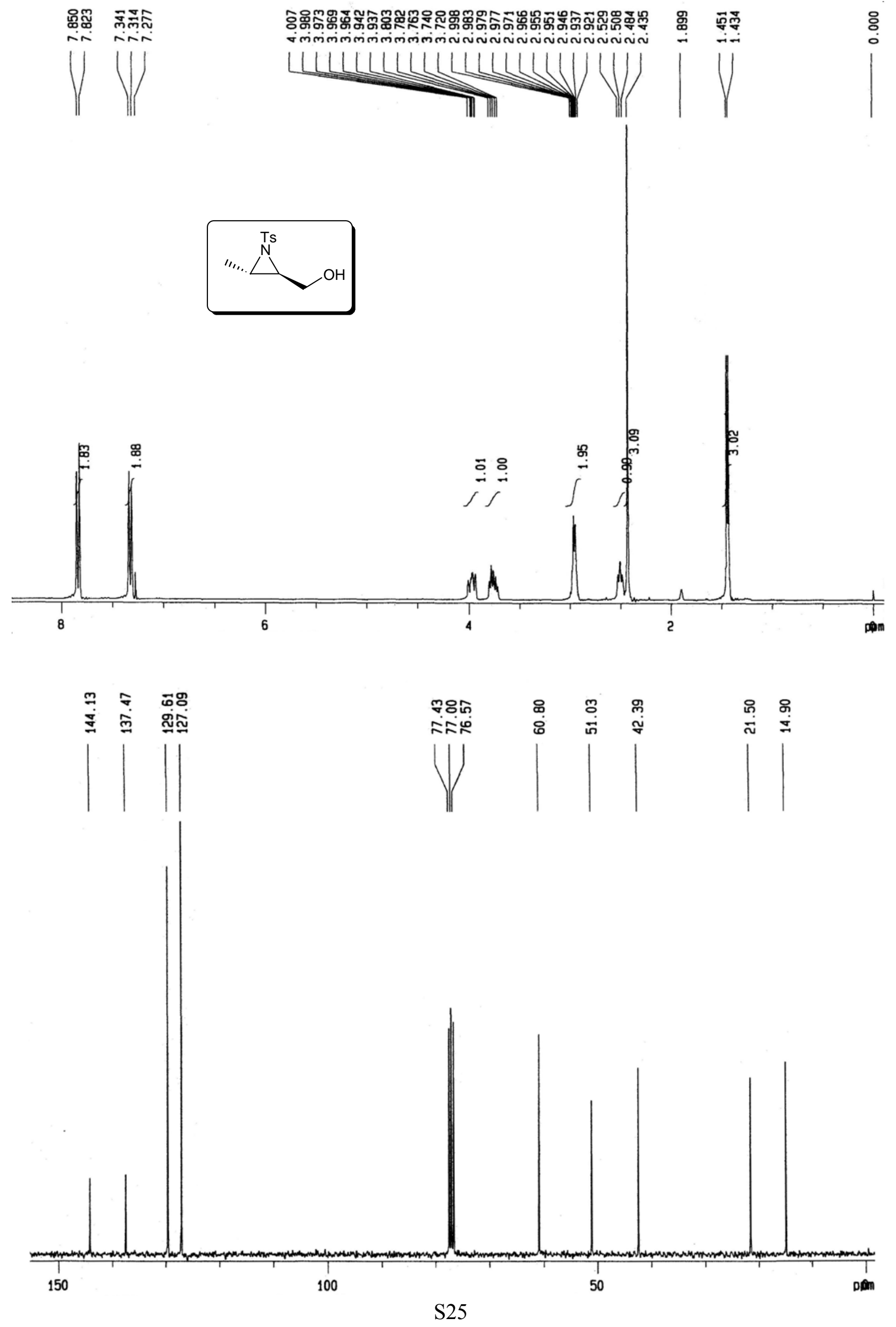



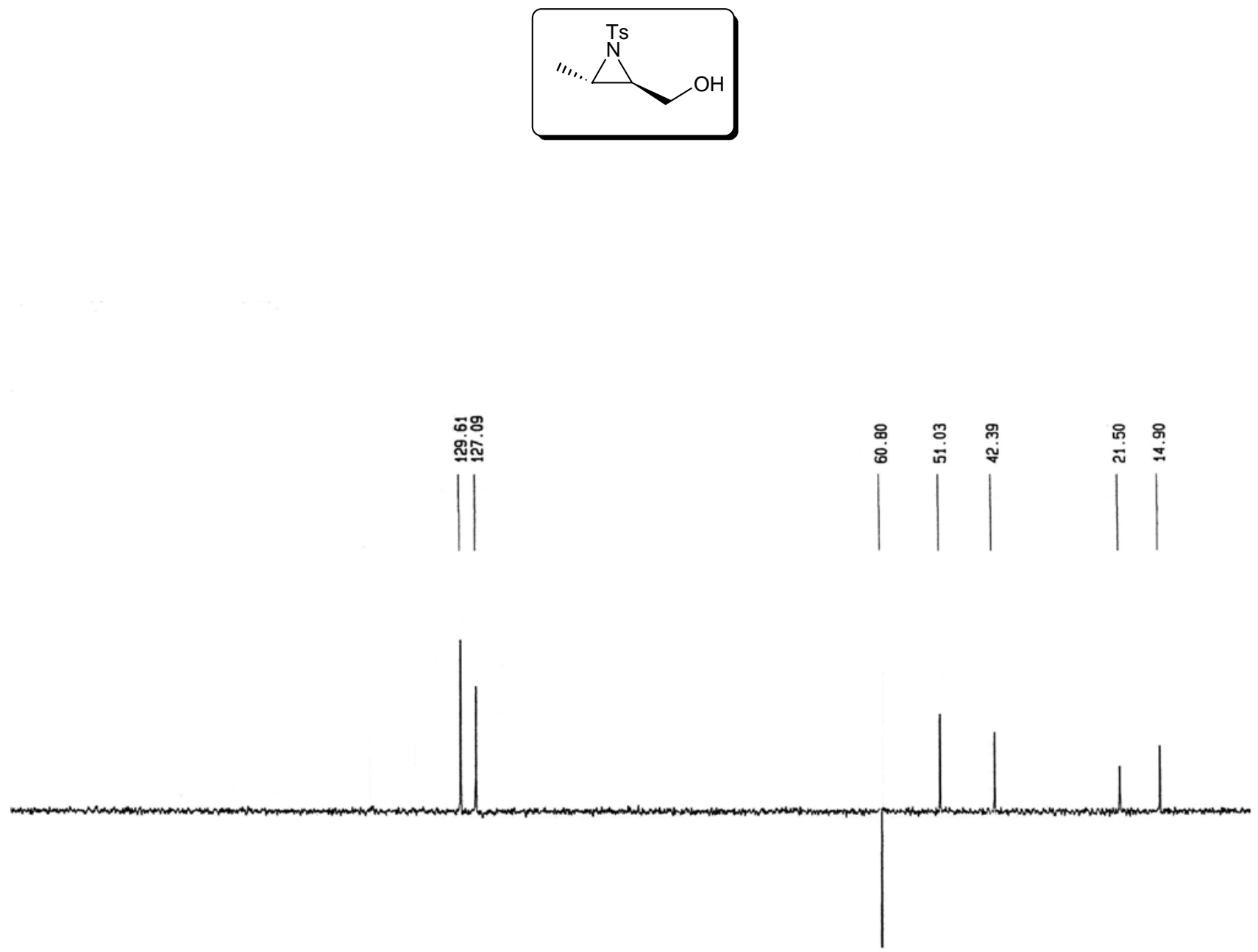

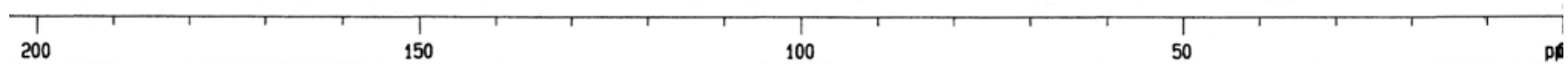




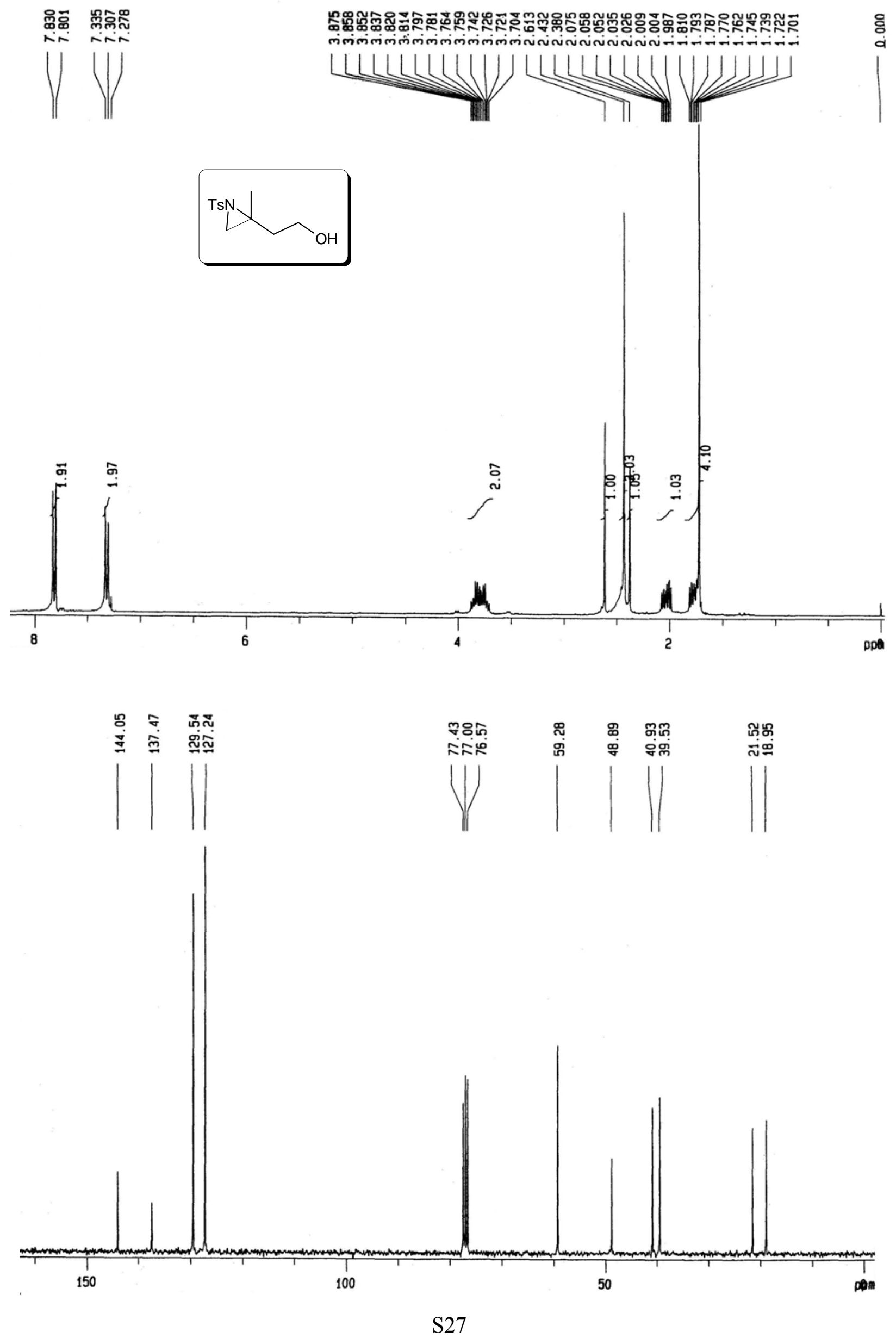



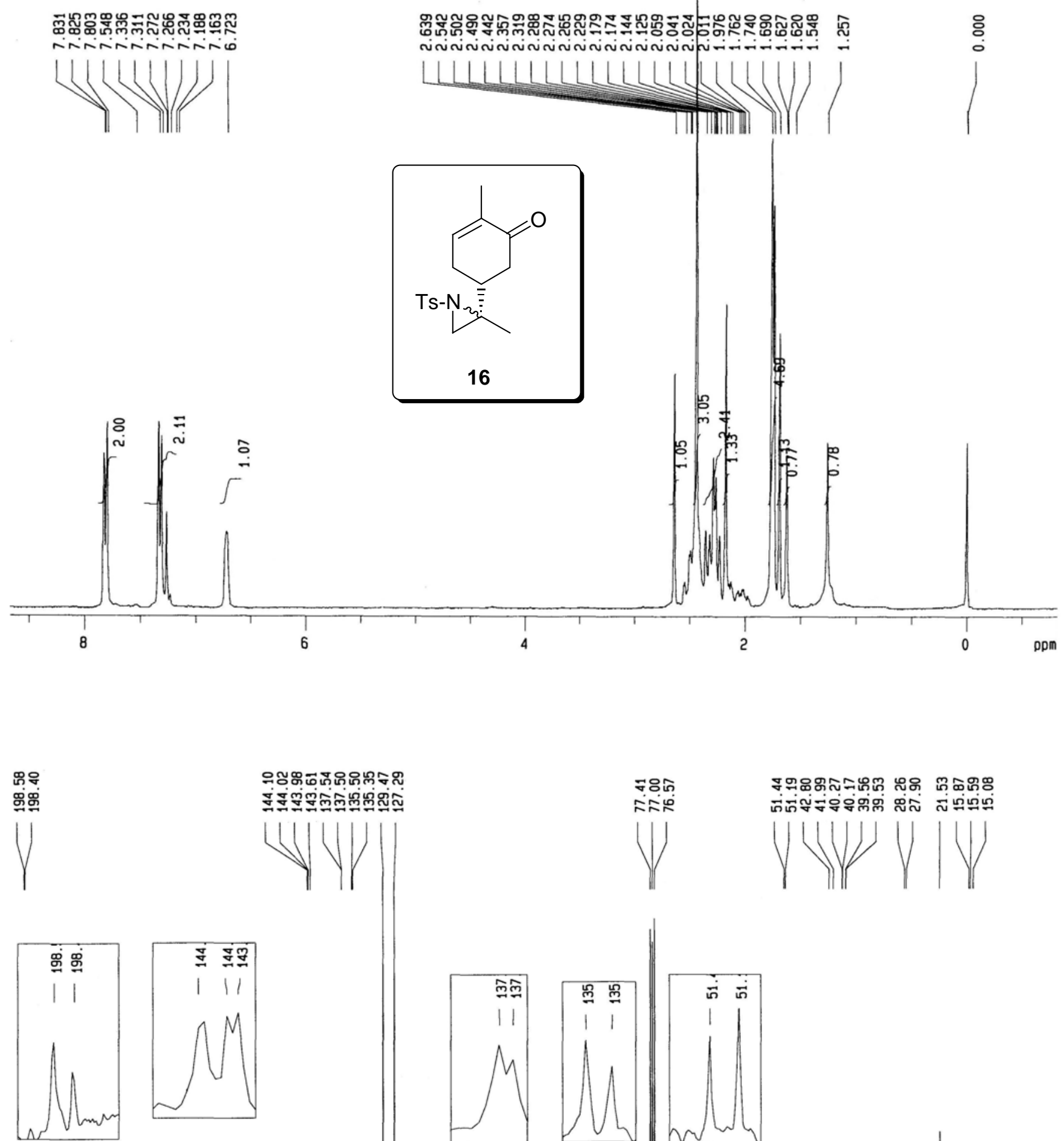

우ำตี

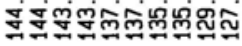

Fotก

중ํㅇ

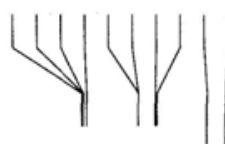

U

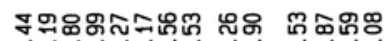

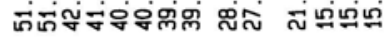
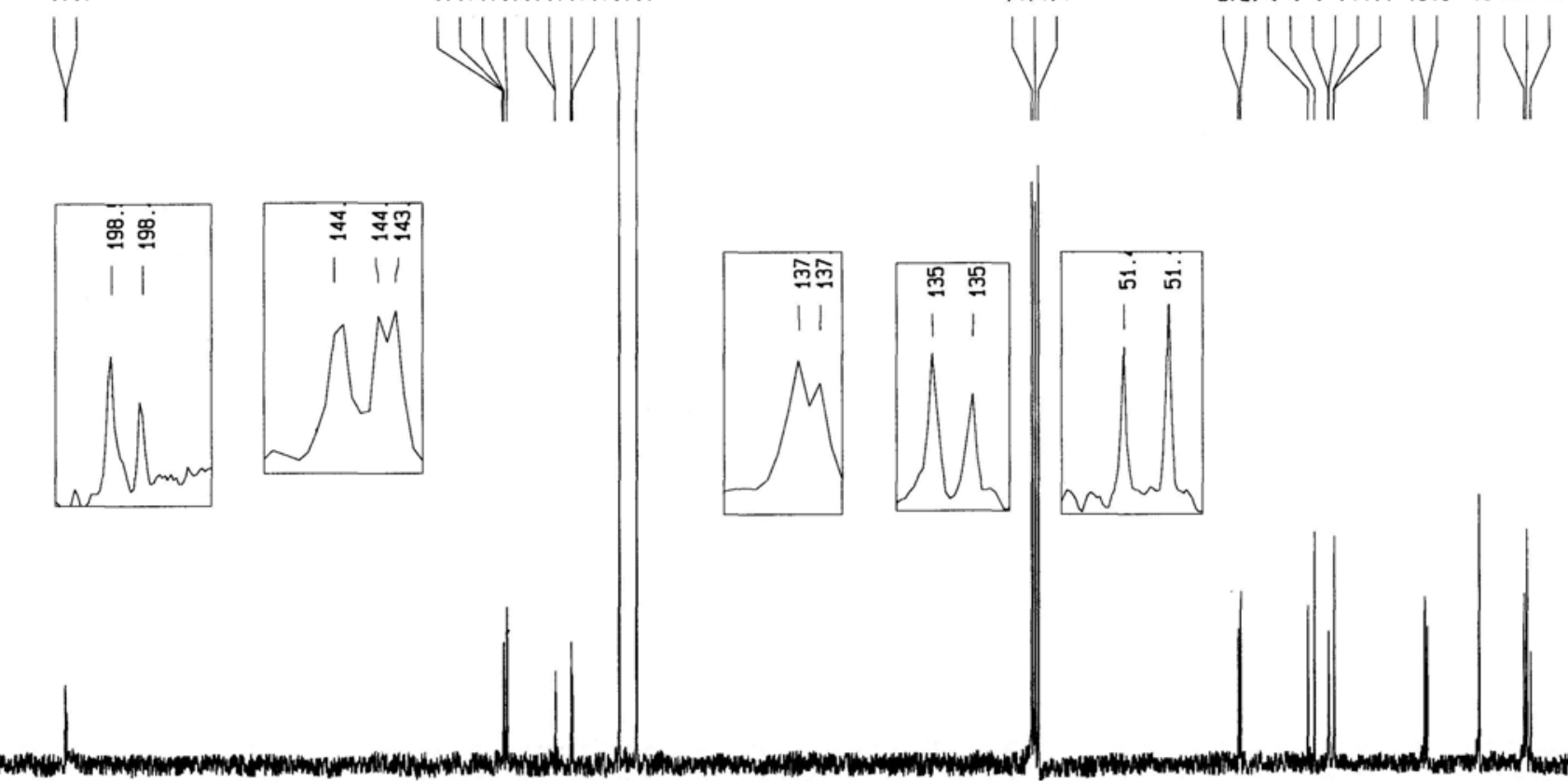

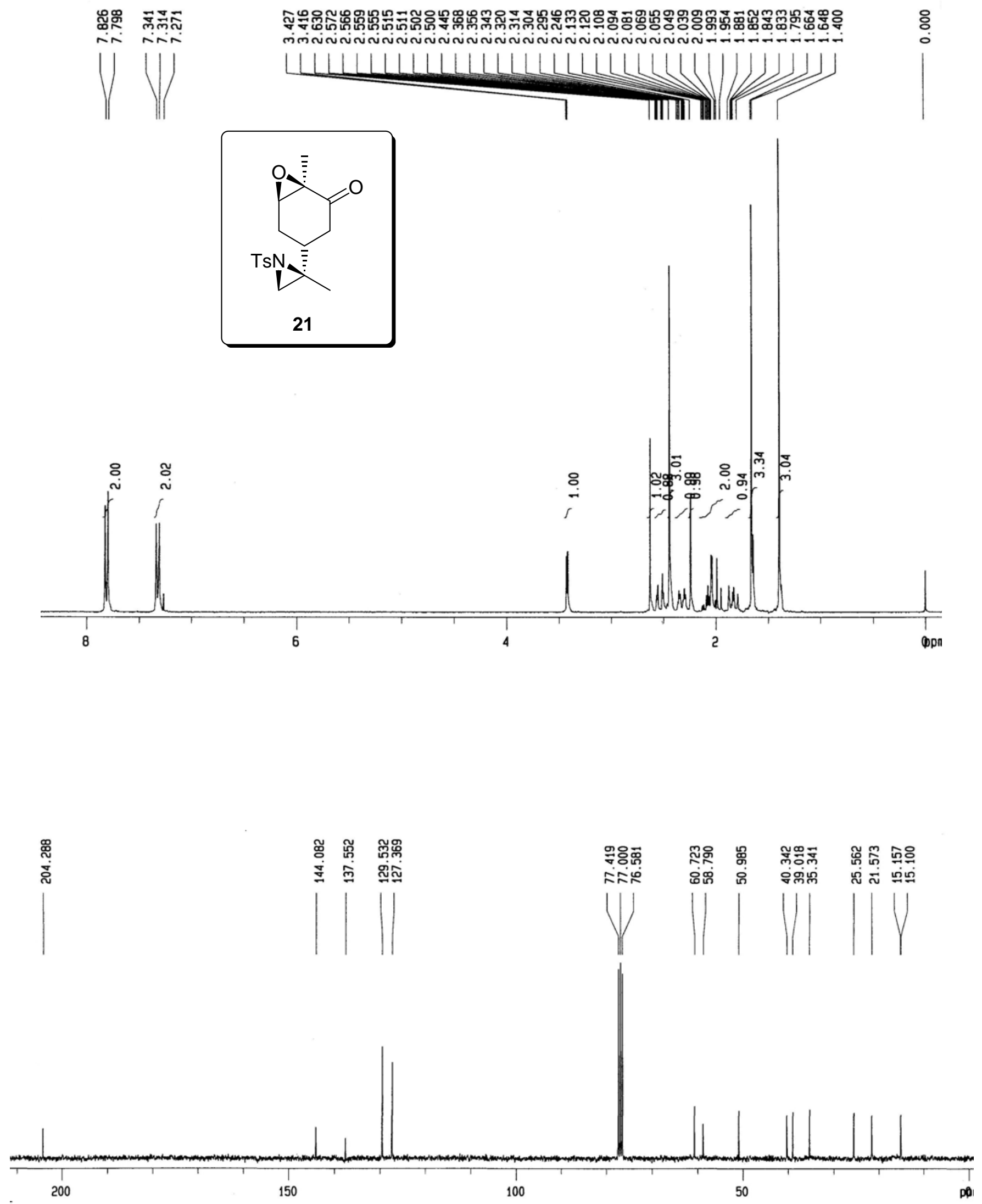

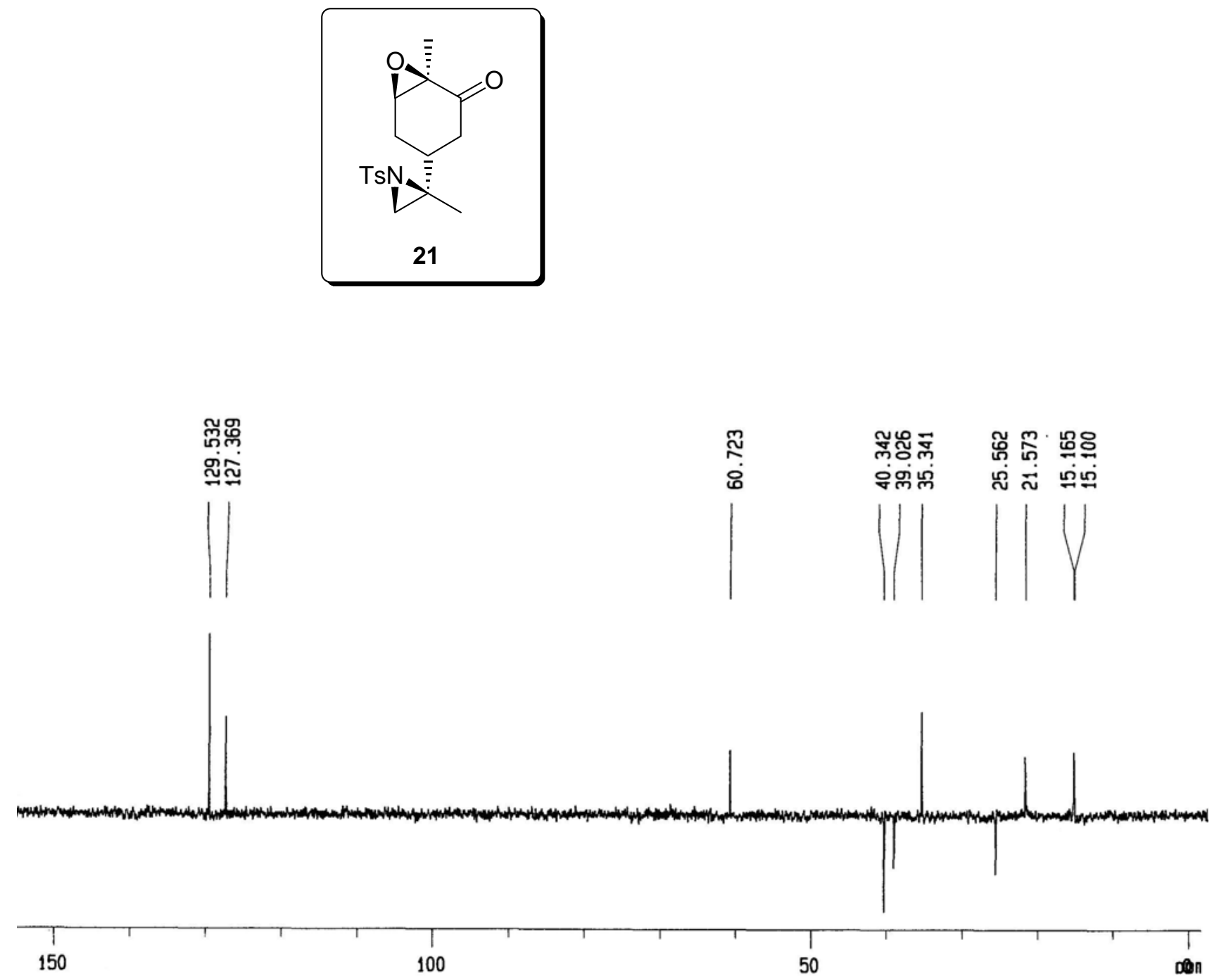

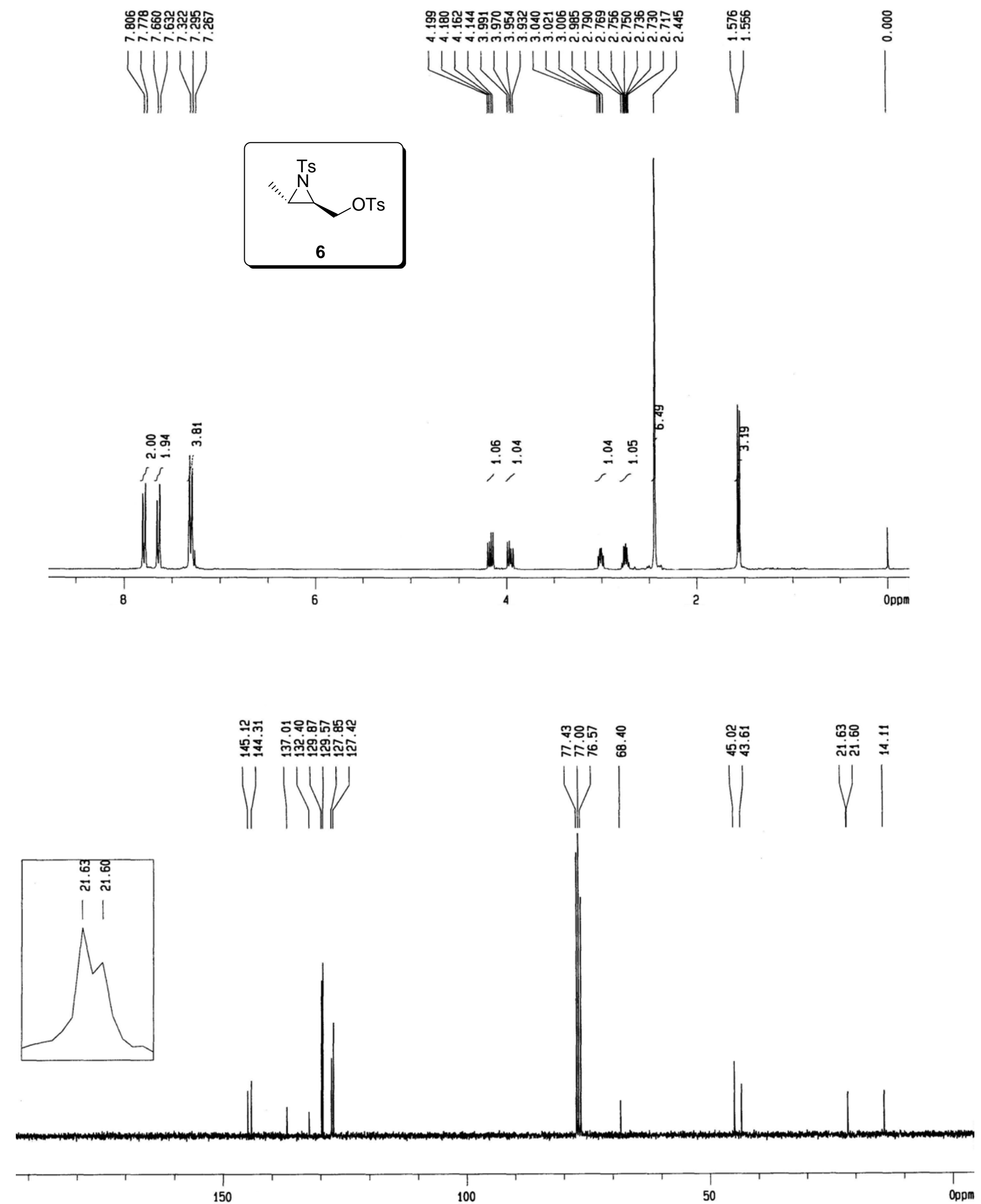

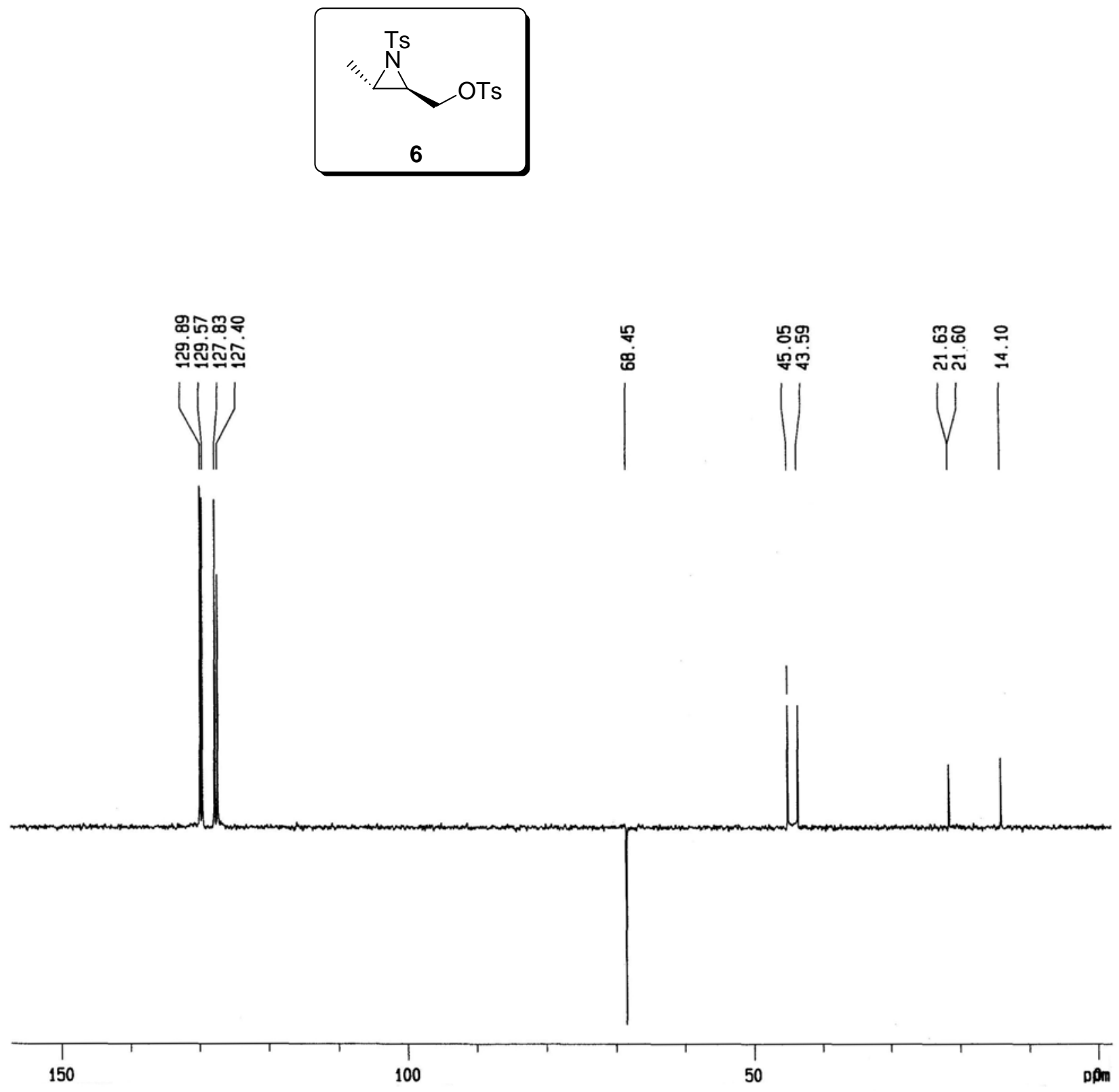

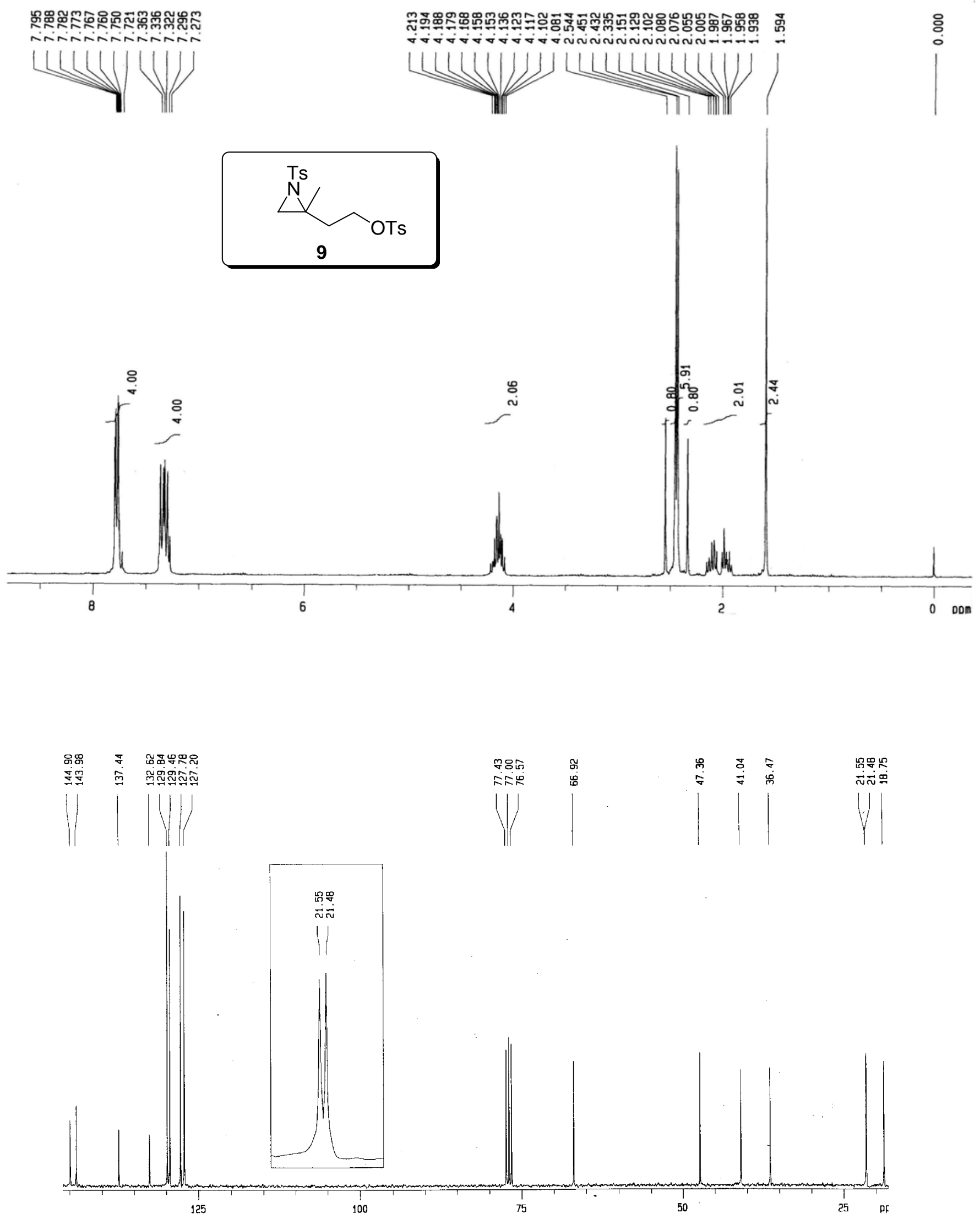

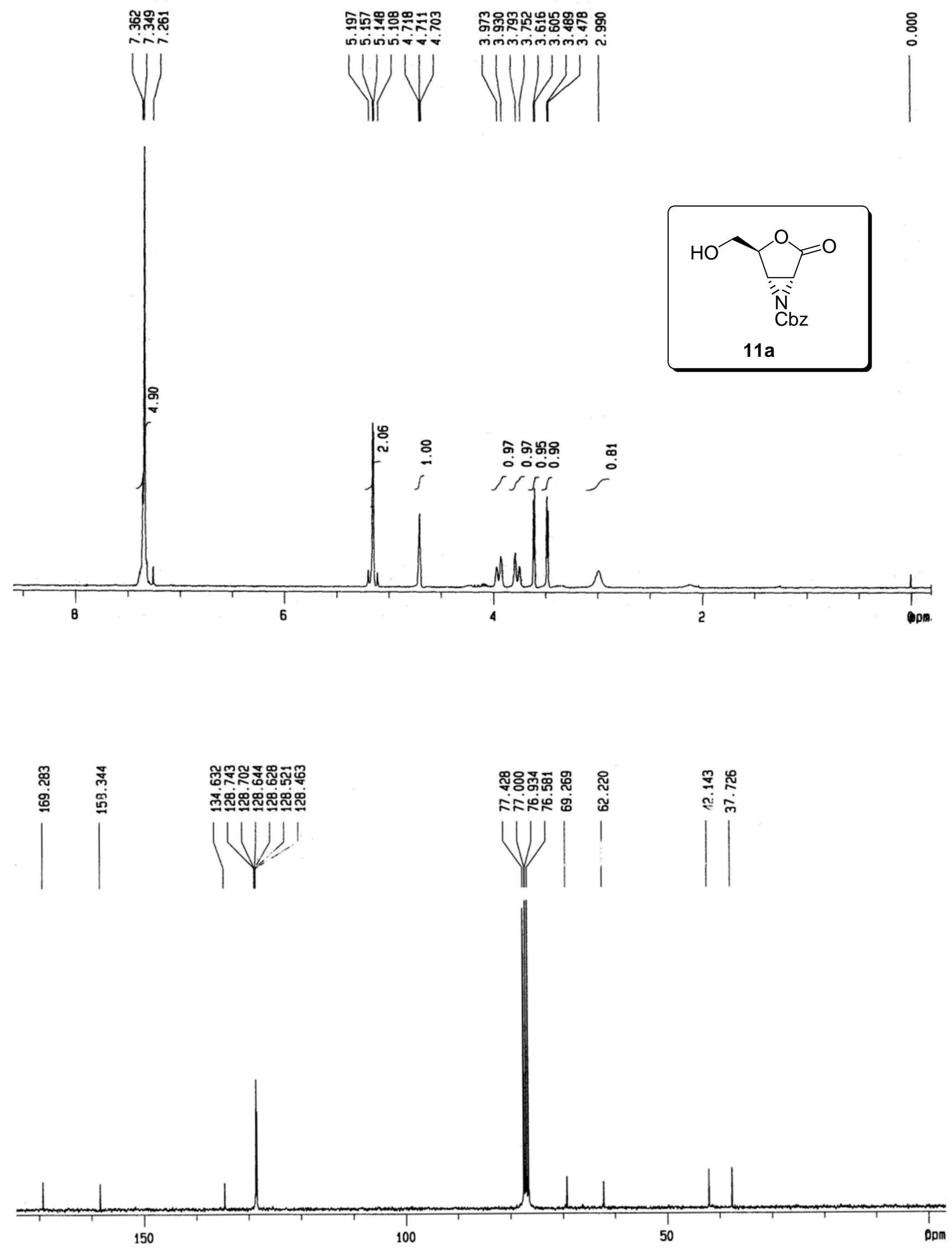

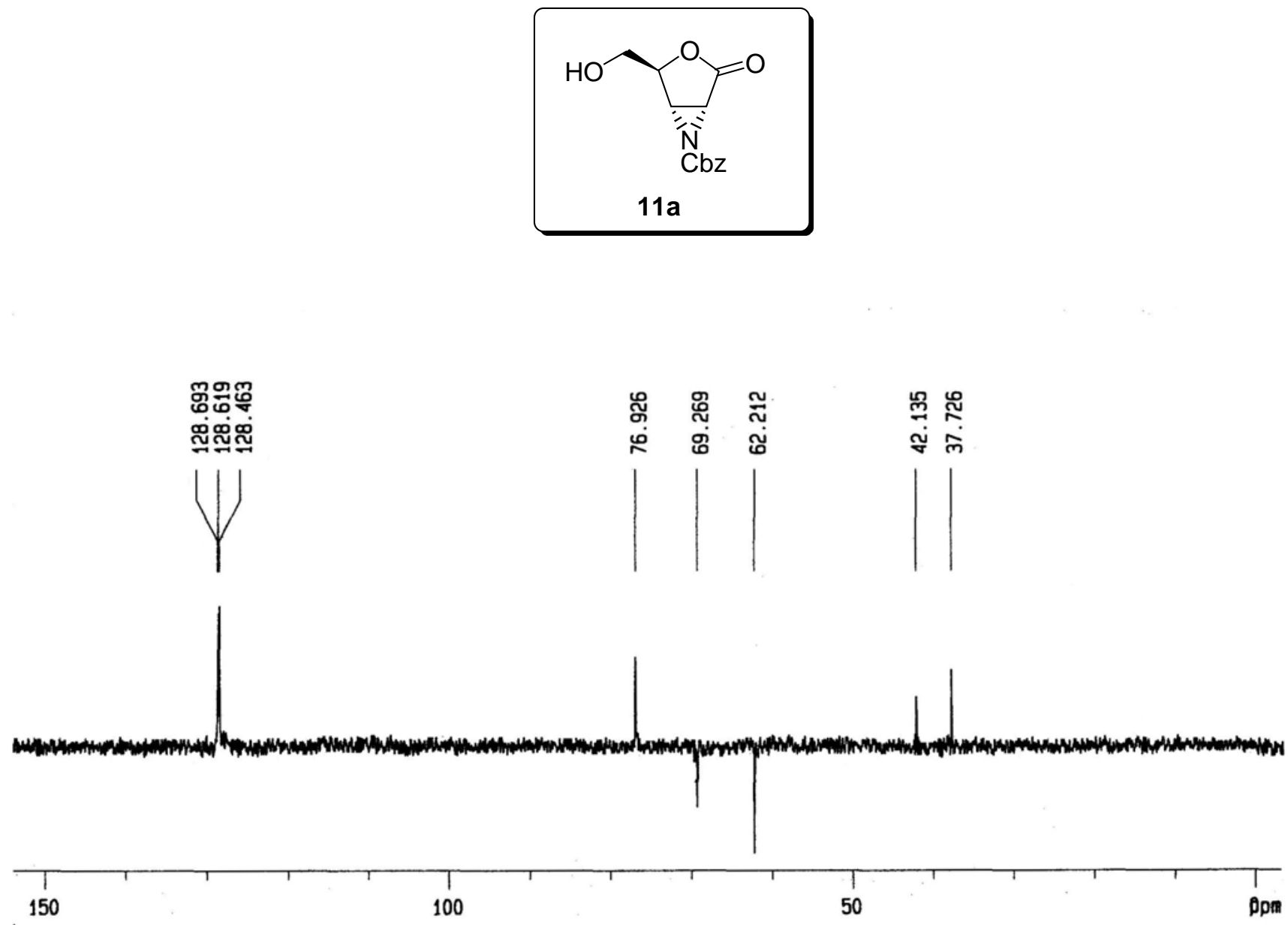

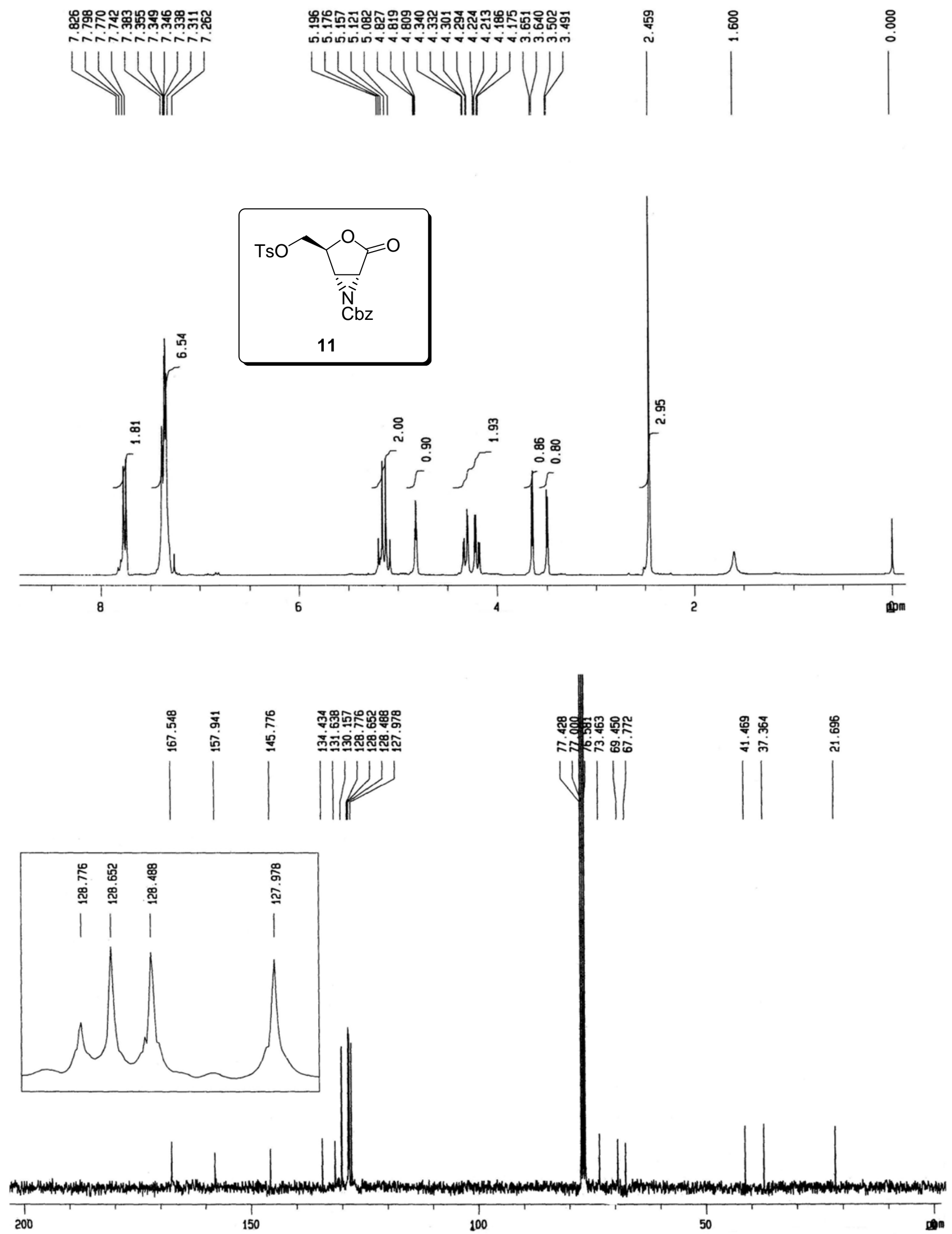


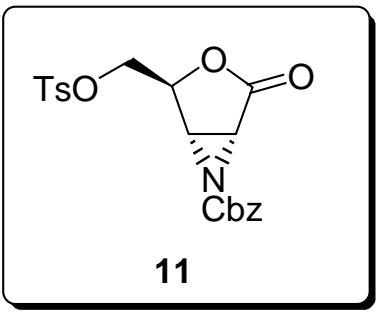

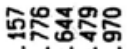

ำㅉำㅉำ

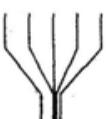

草视

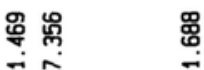

ก เต่อ
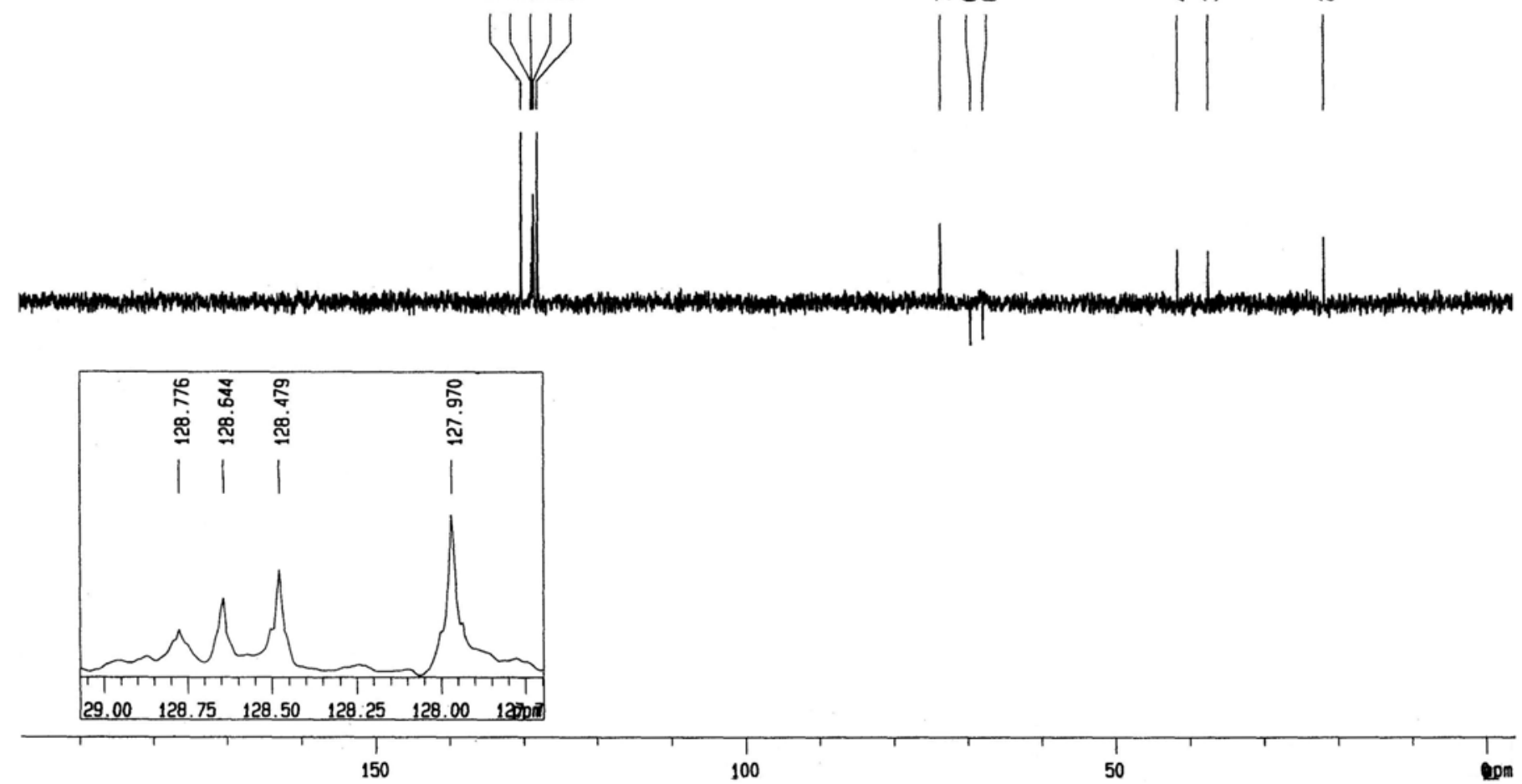

$\begin{array}{llllll}29.00 & 128.75 & 128.50 & 128.25 & 128.00 & 1270 \text { a }\end{array}$ 

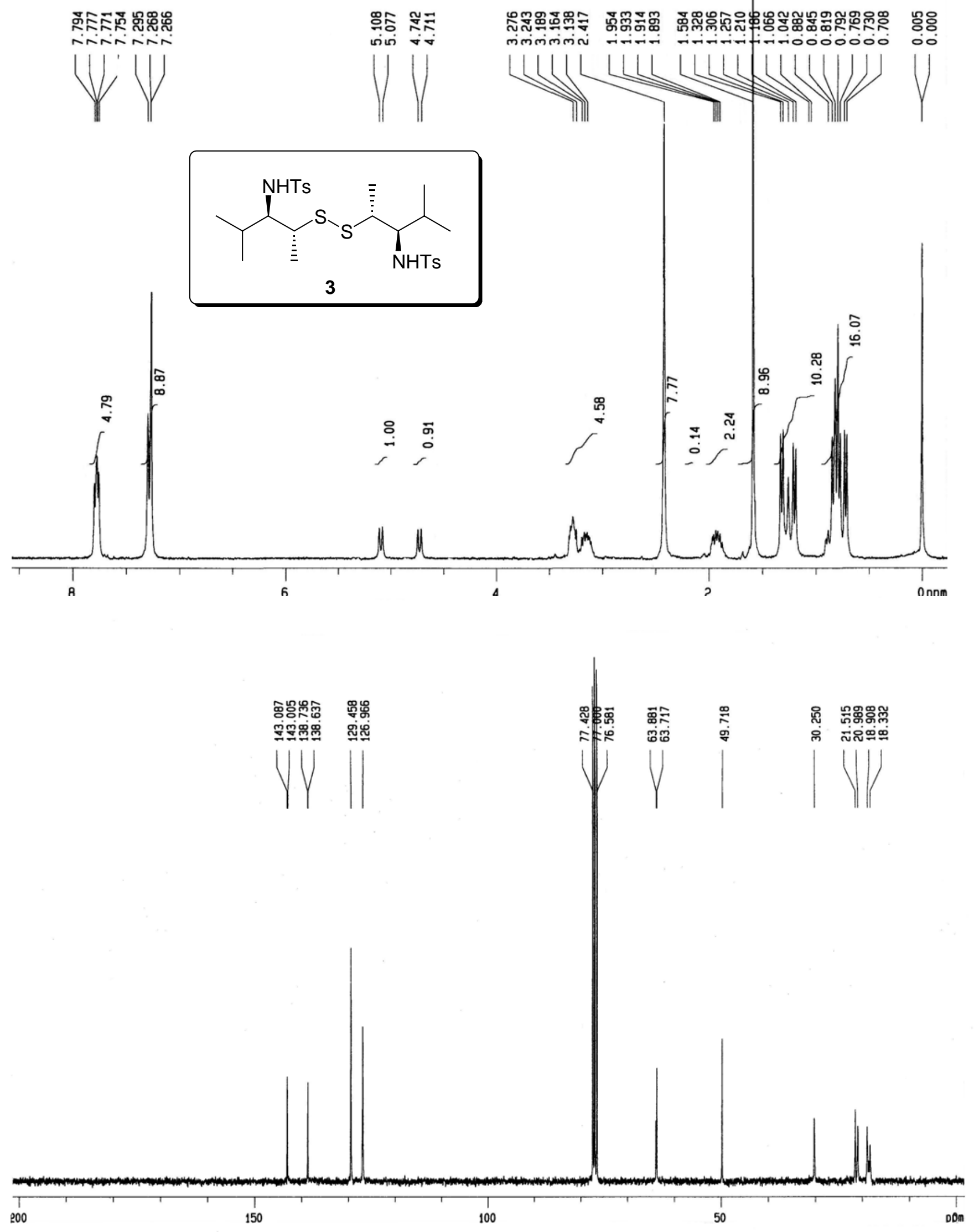


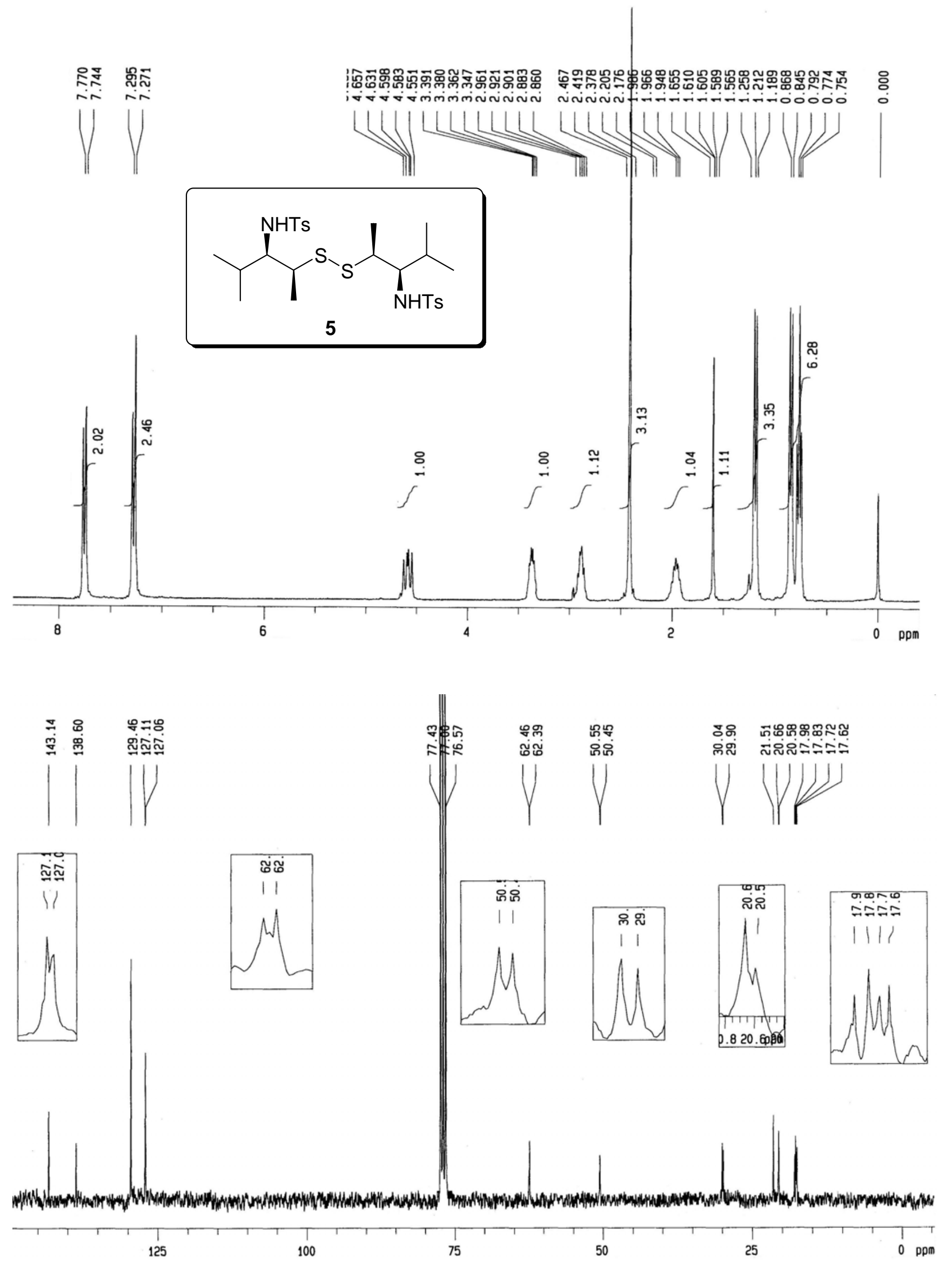



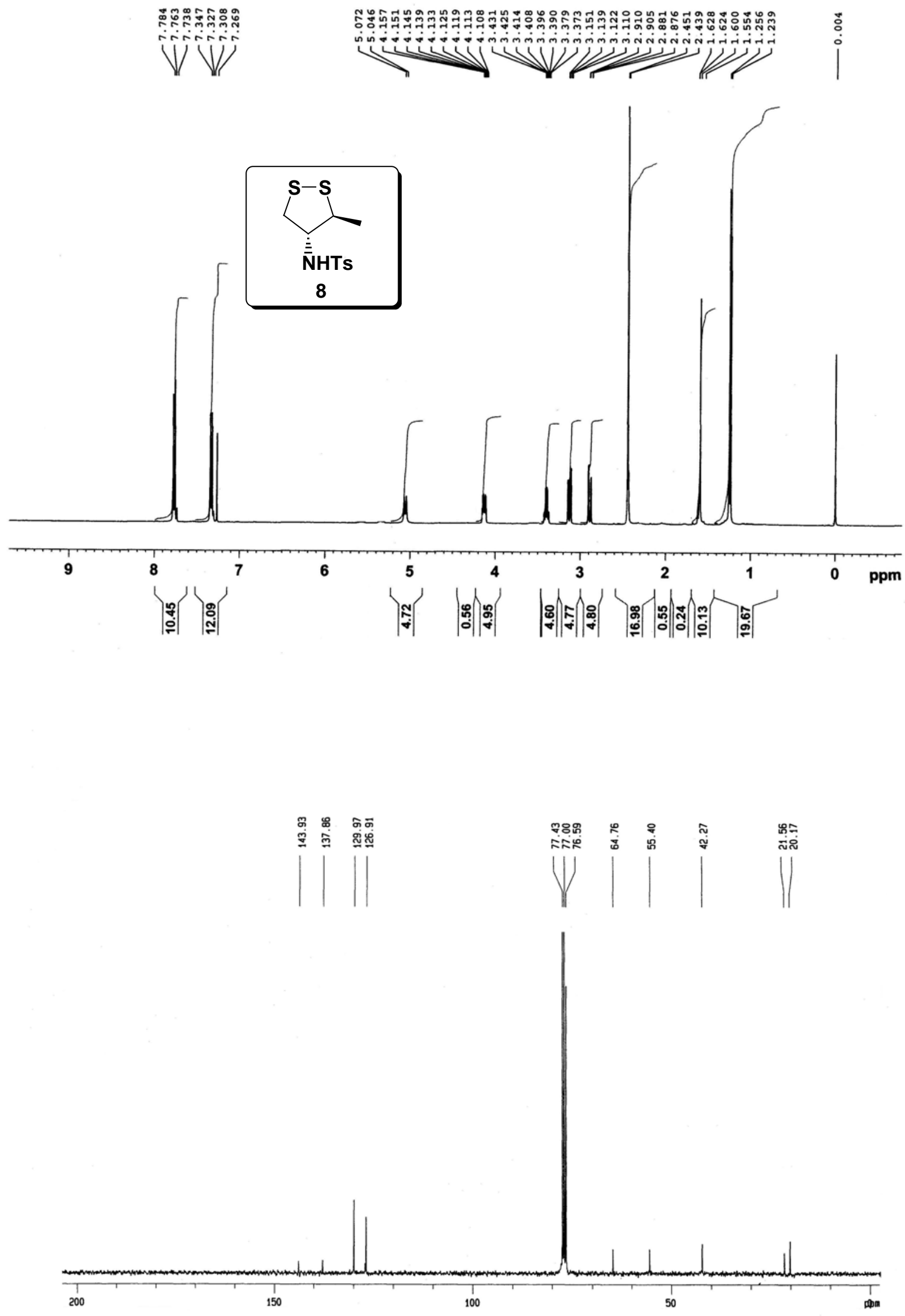

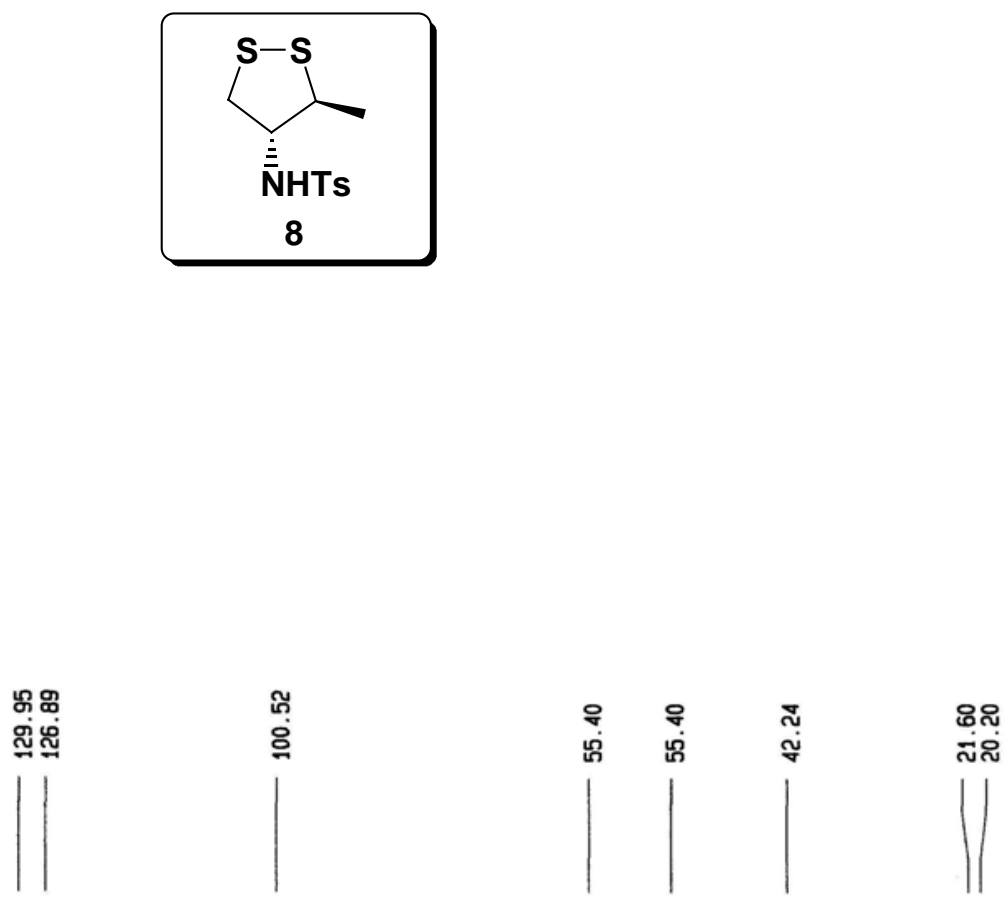

|l

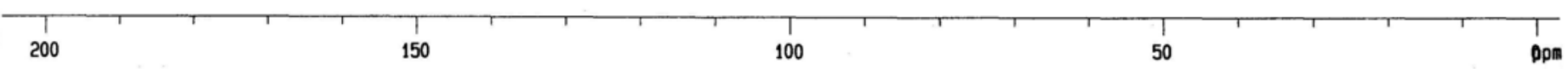




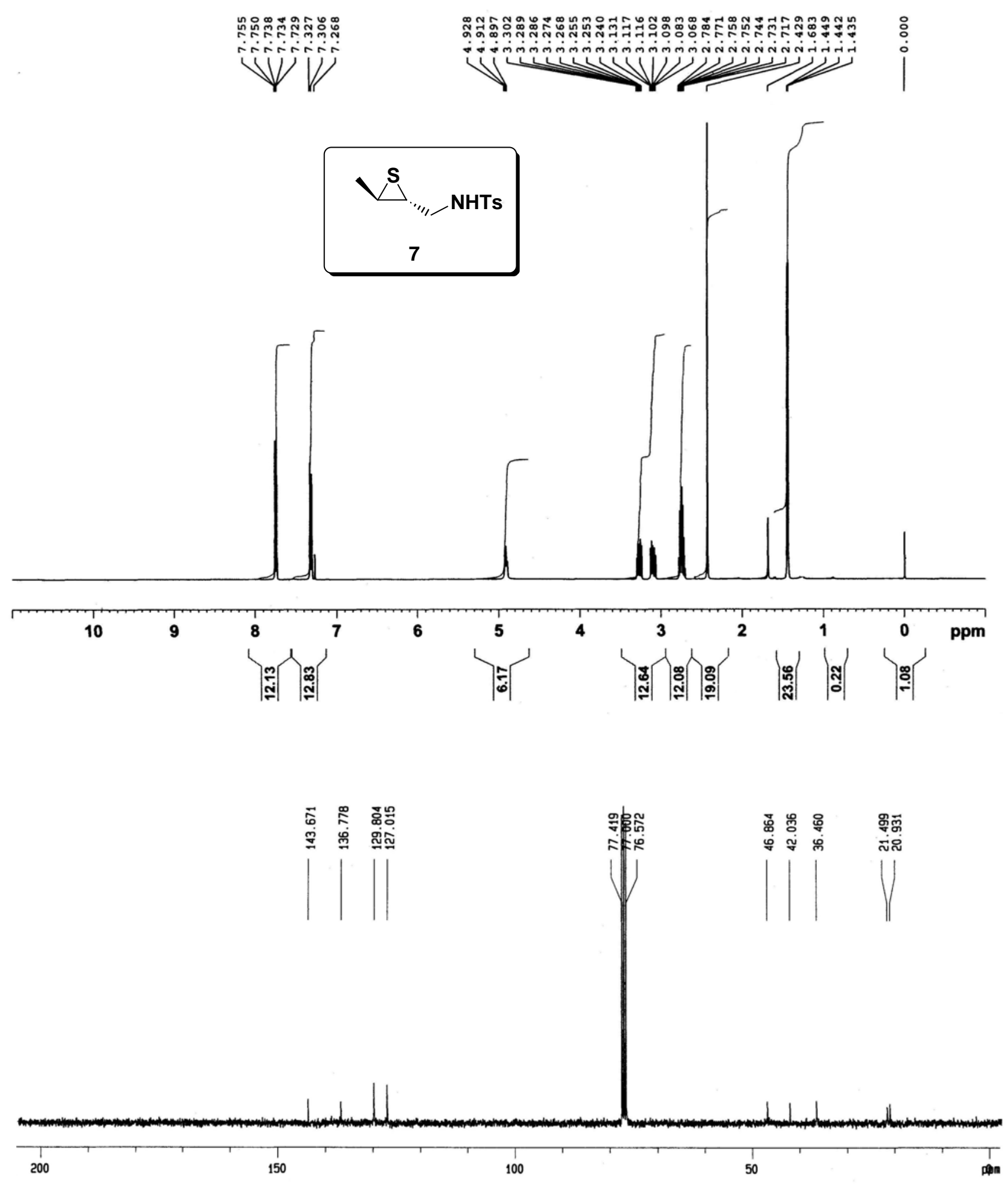




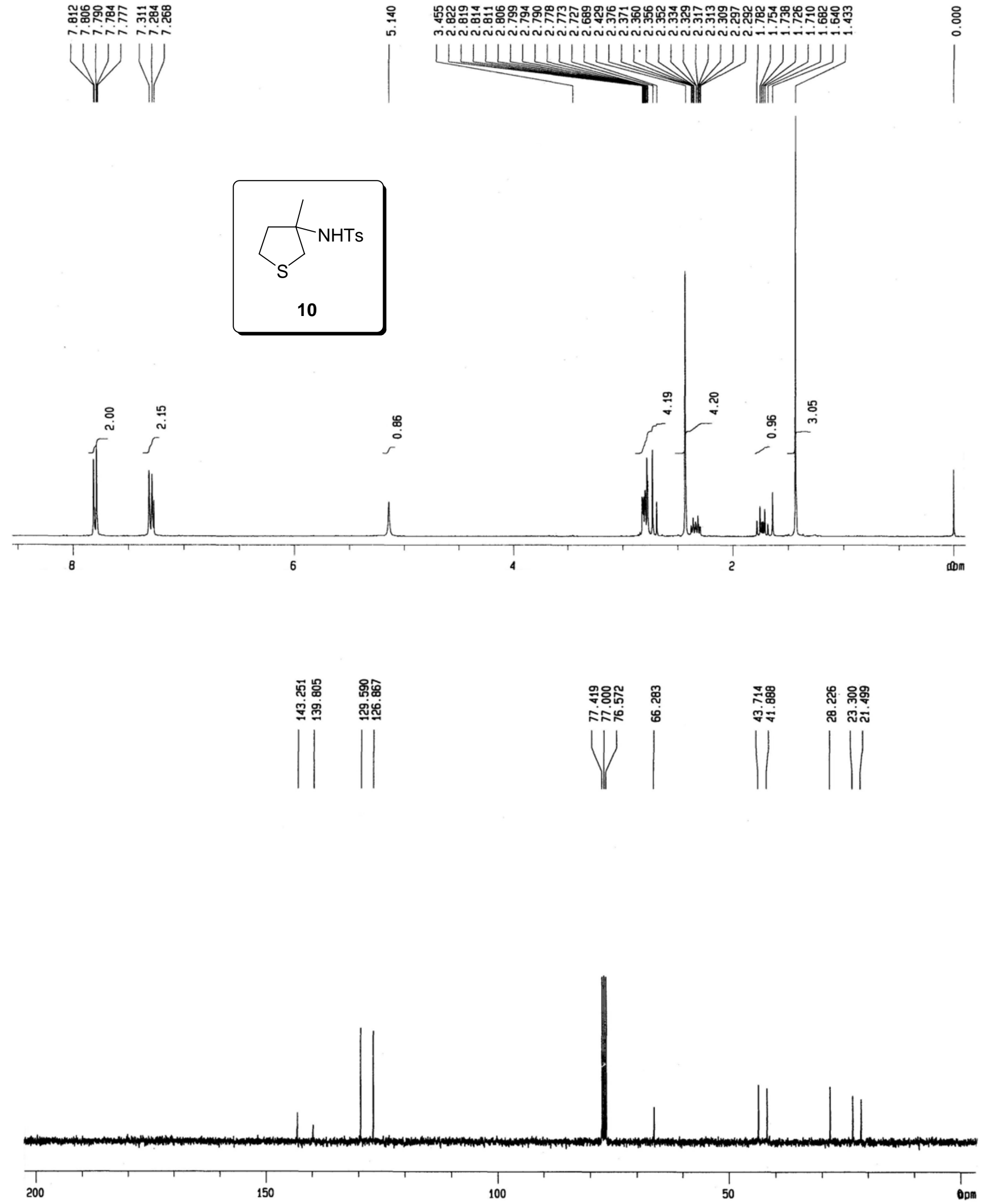



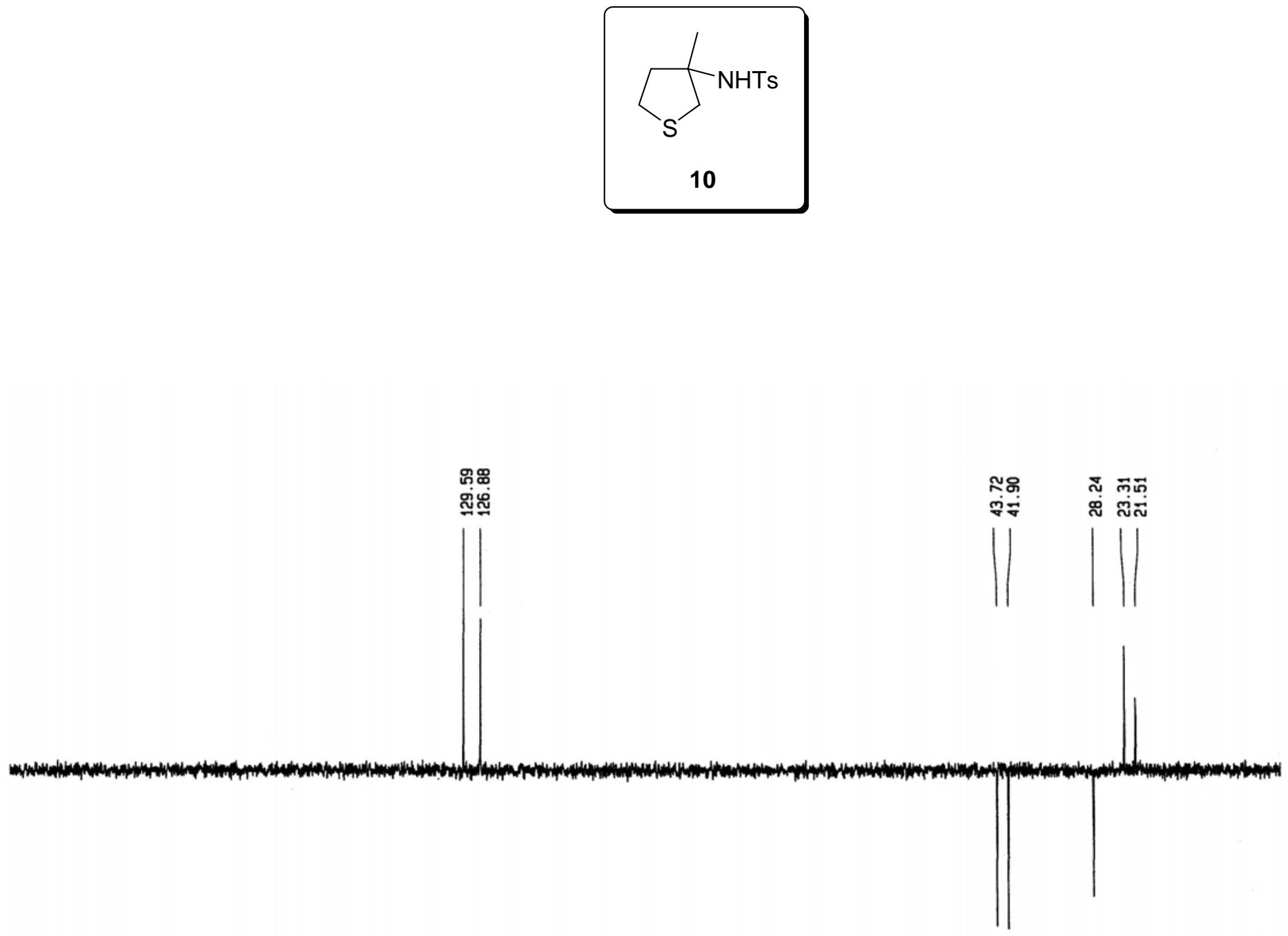

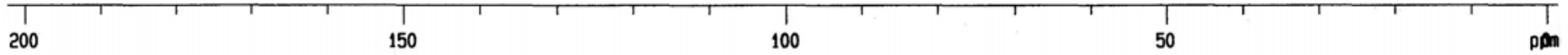


구ㅇㅛㅛ

ininisinis

u. 11111

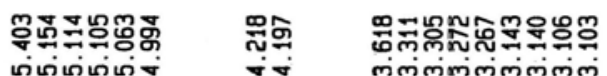

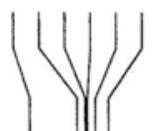

|

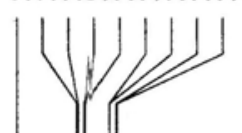

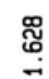

웅
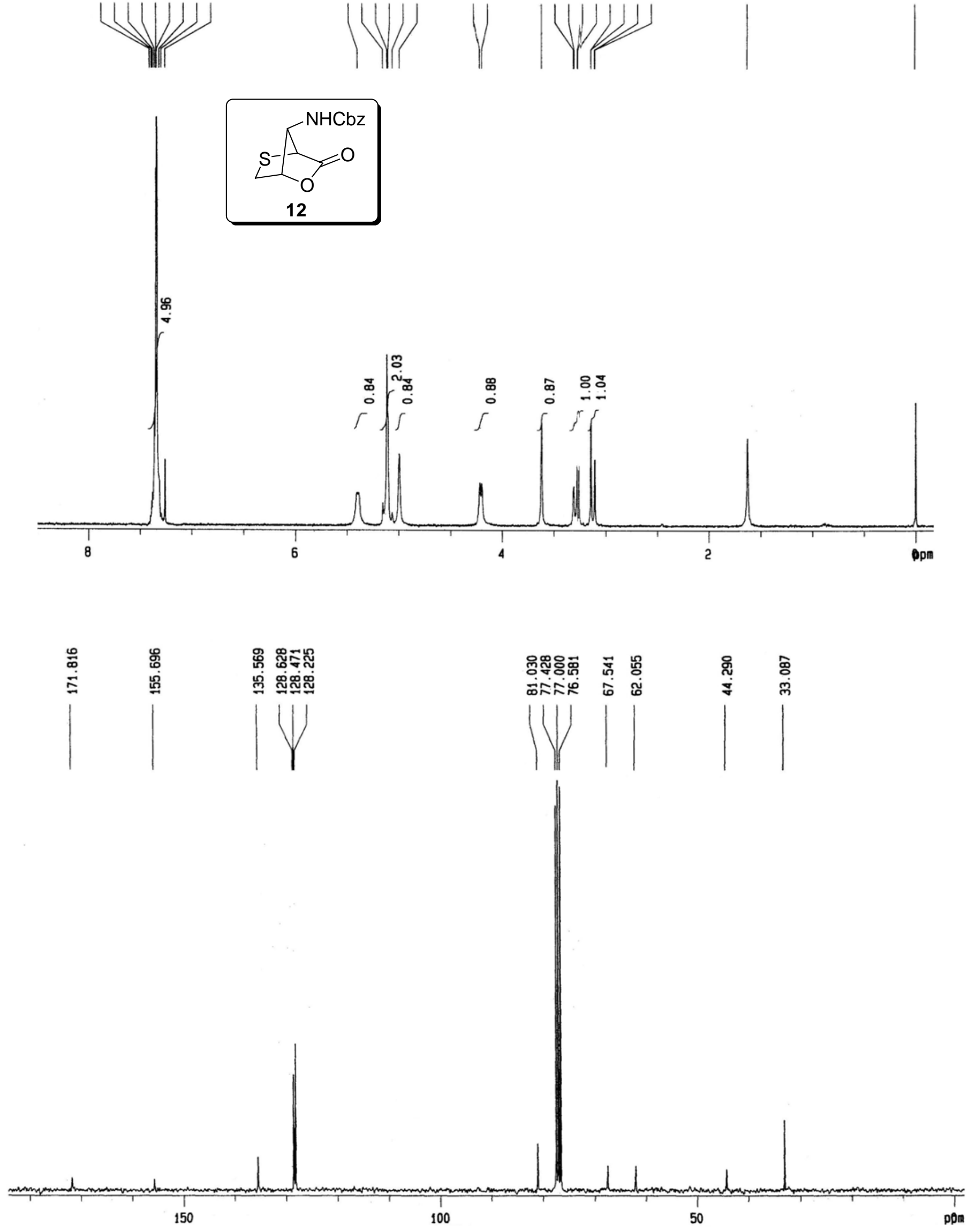

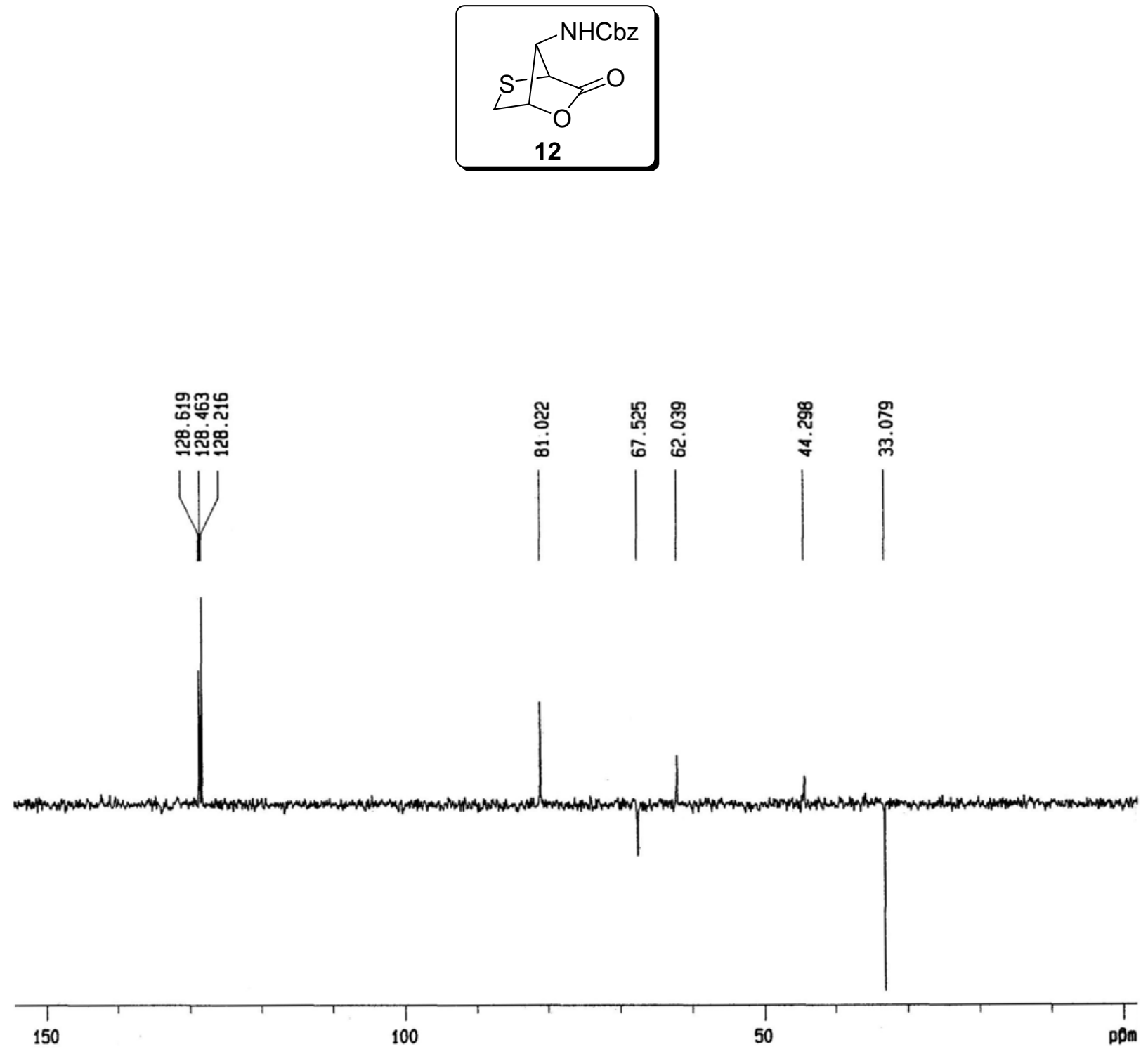

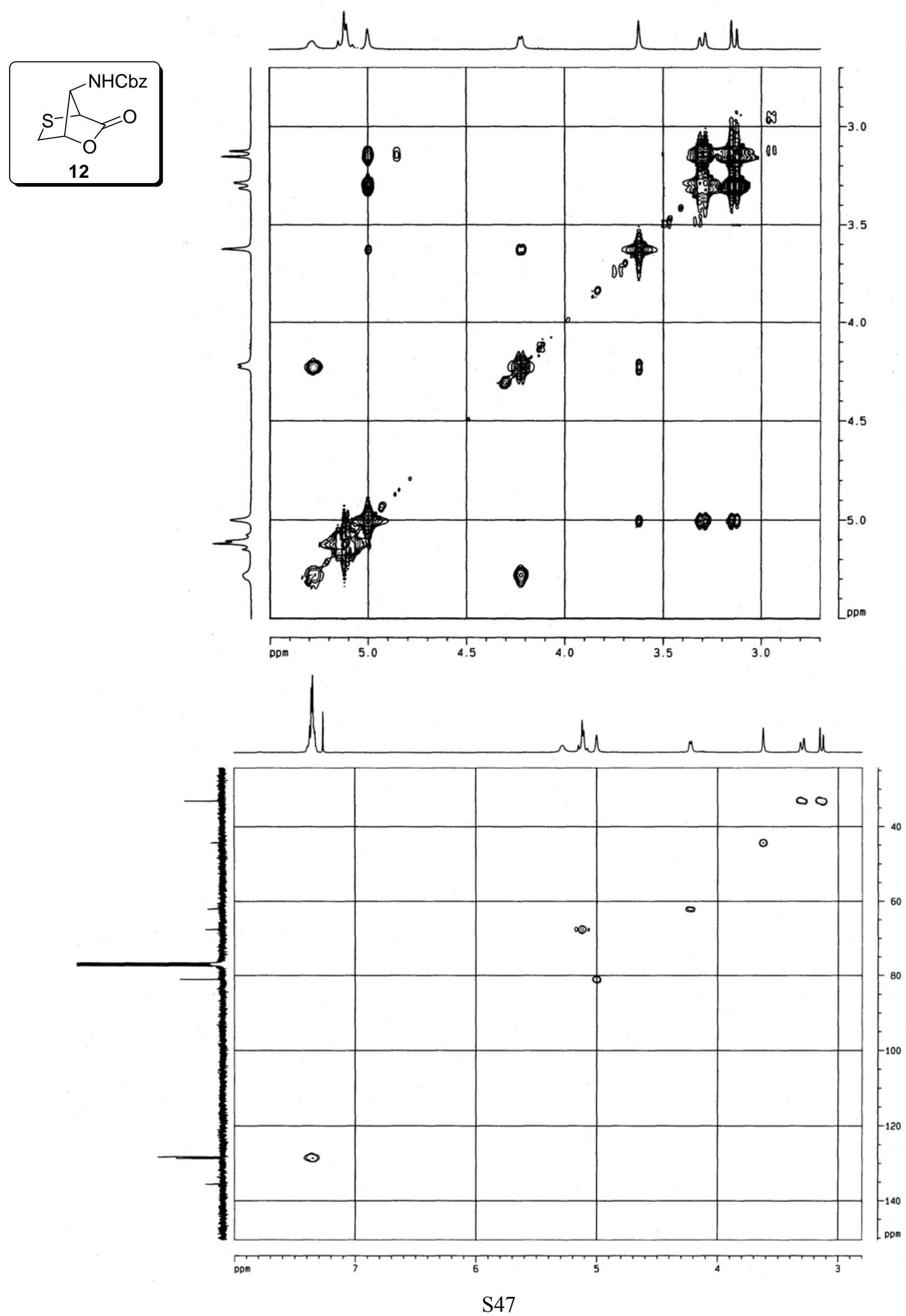

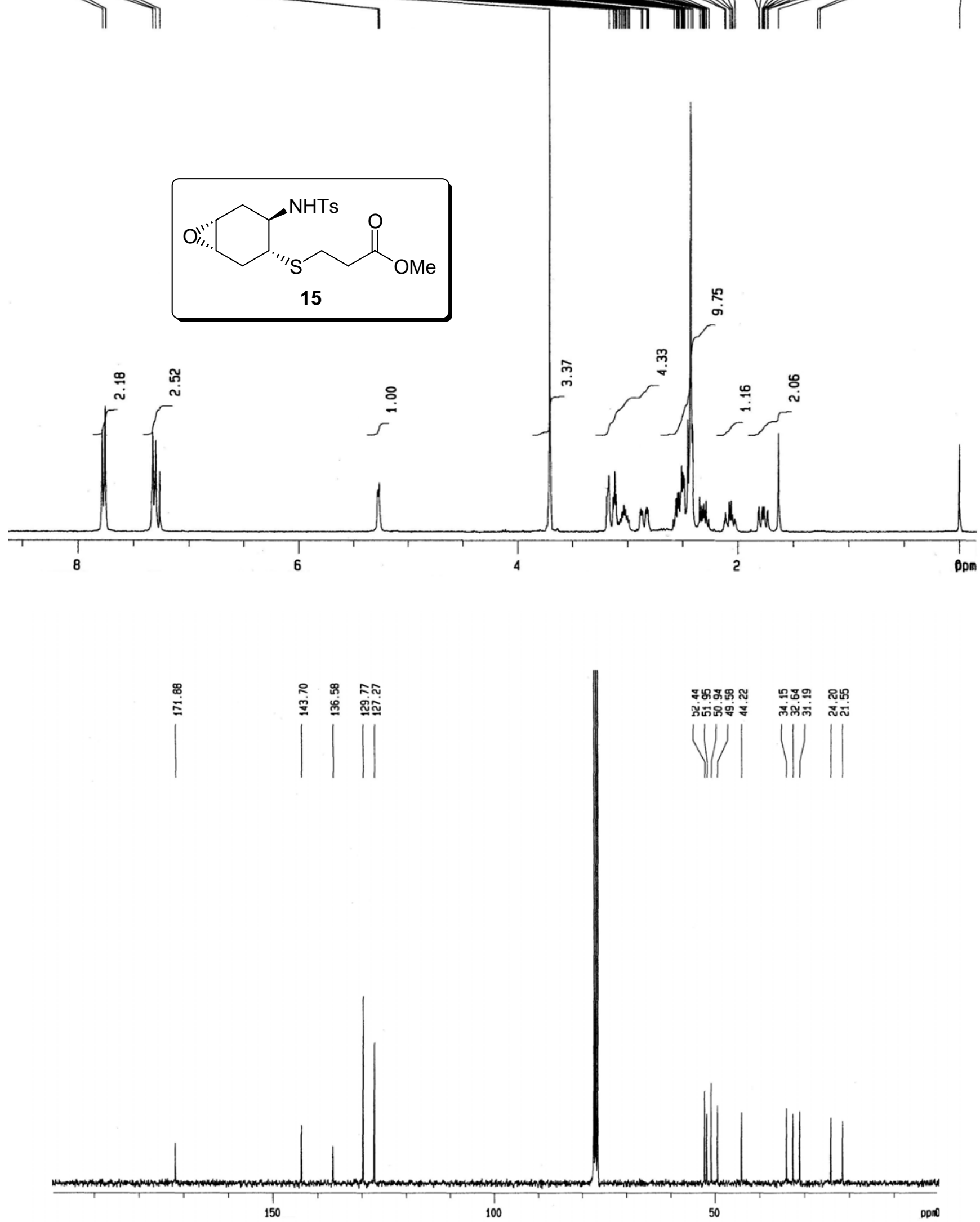


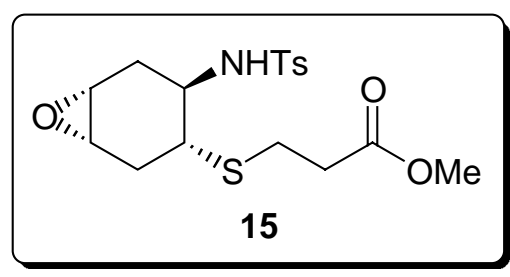

กำก

ูํำ

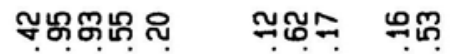

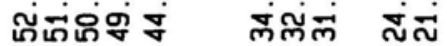

| 1

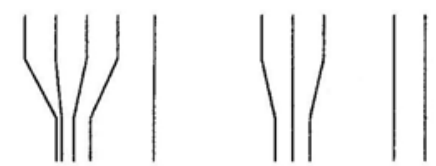

$\mid$

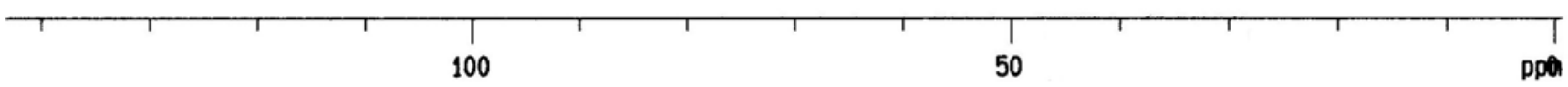



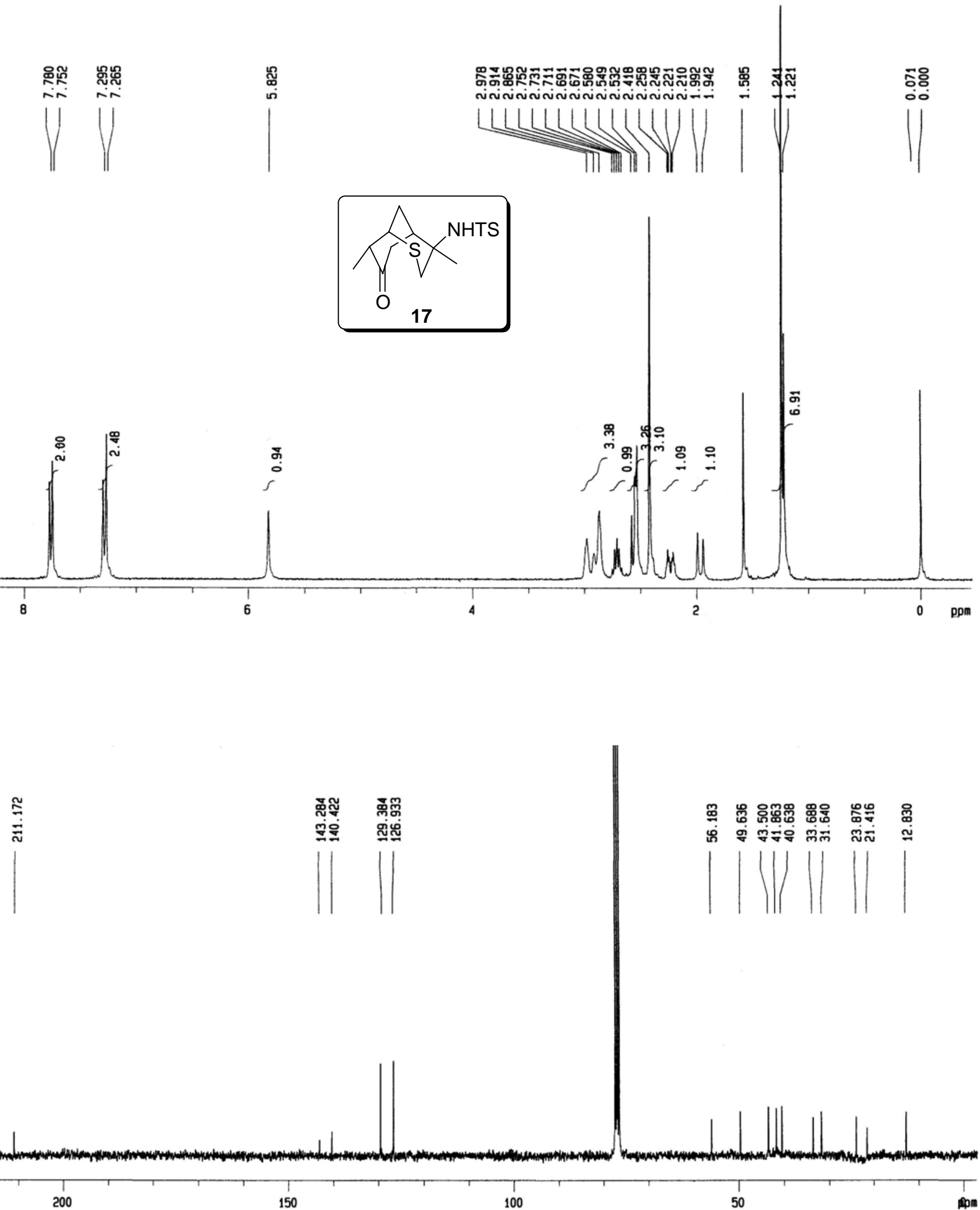

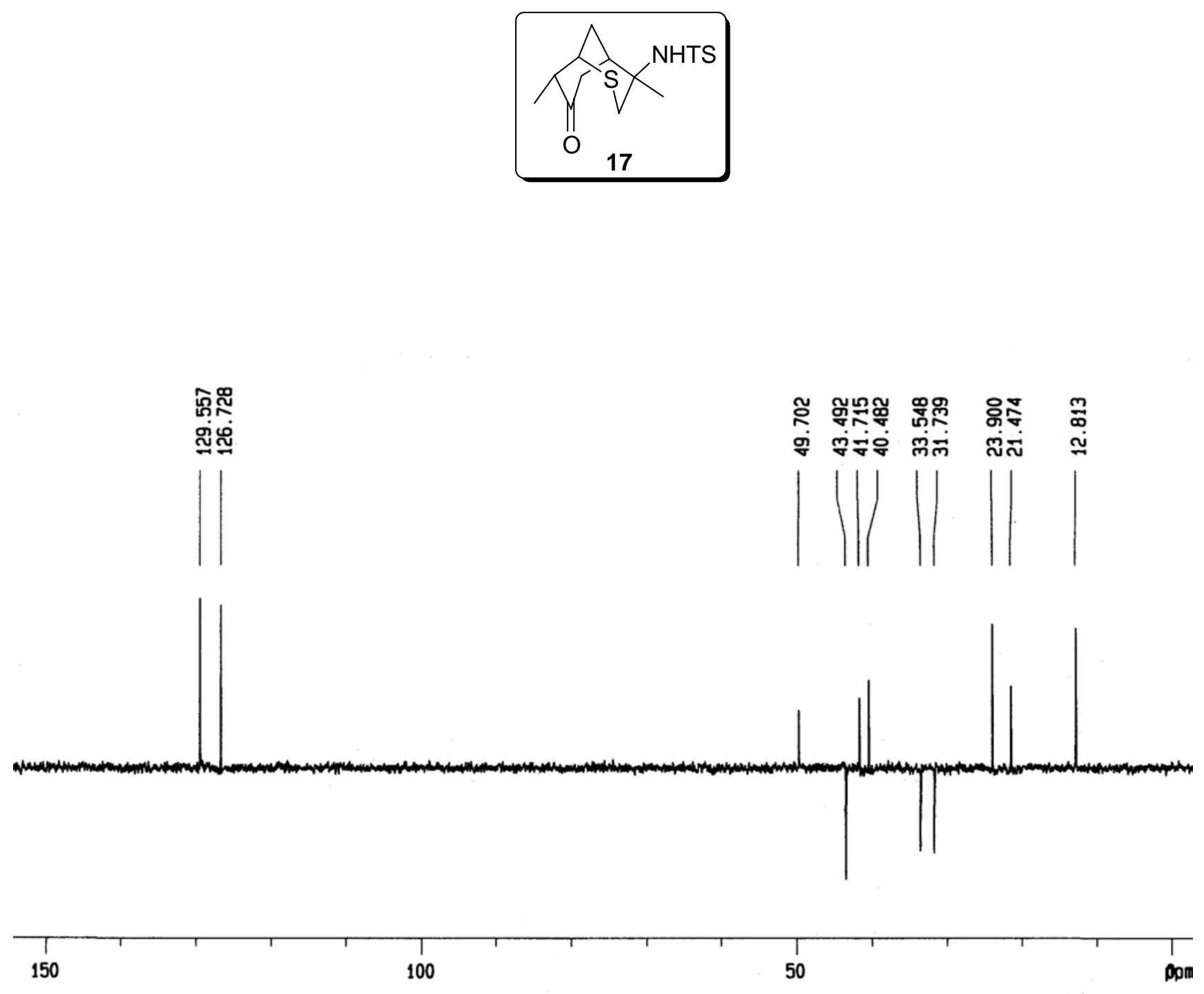

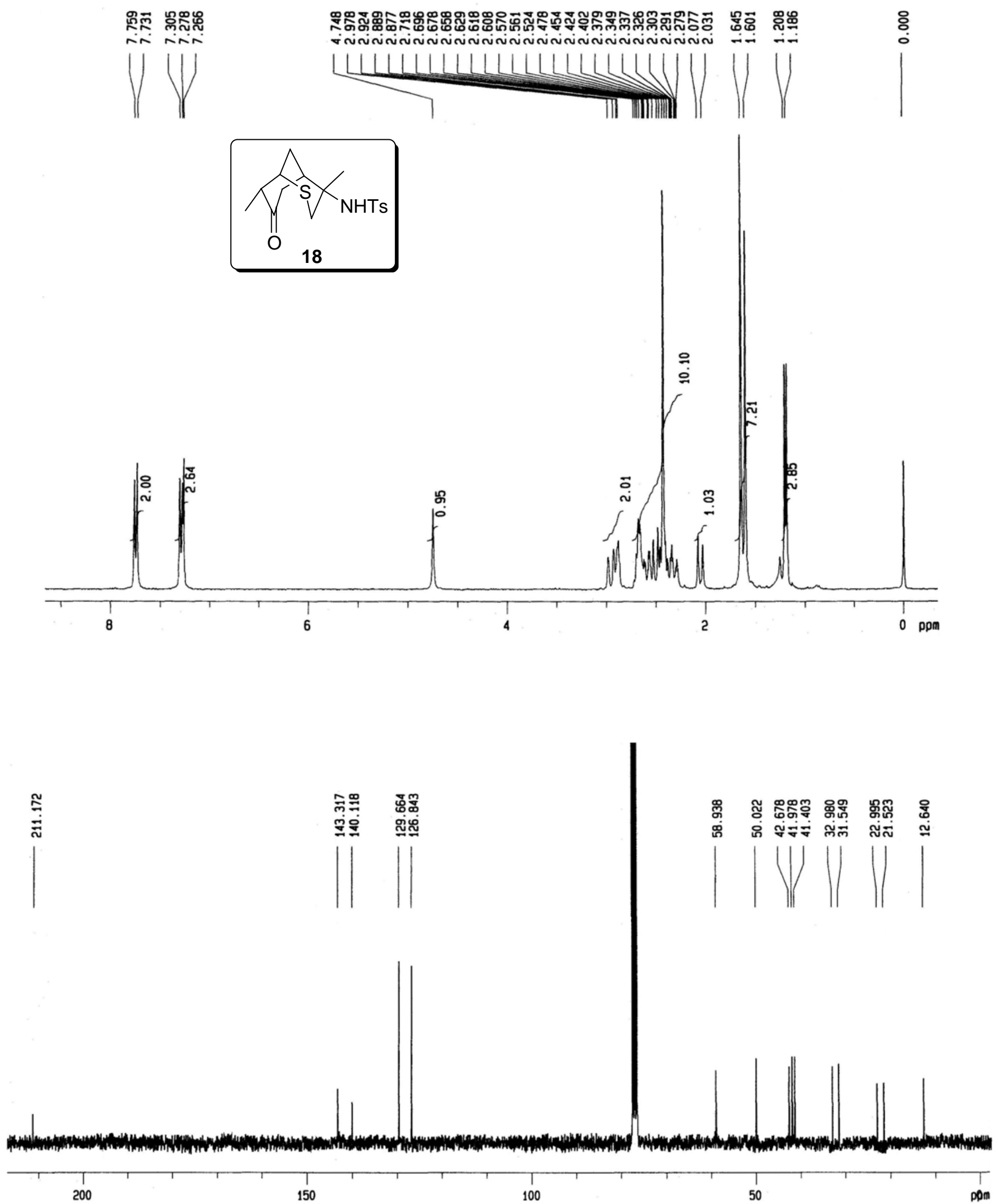

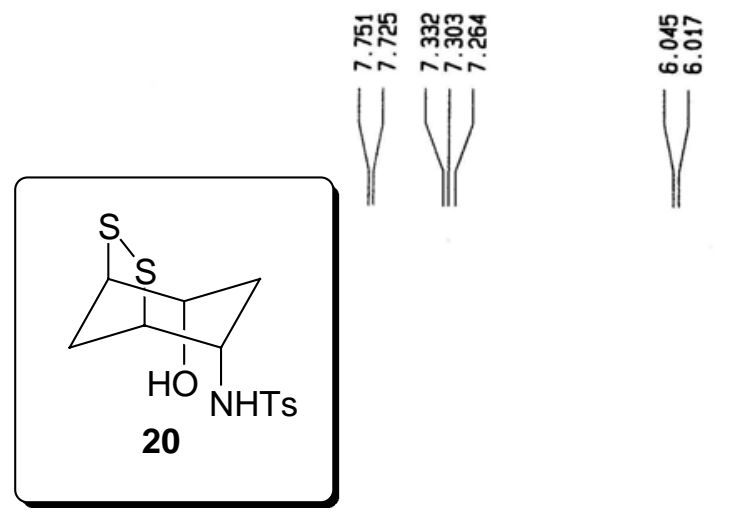

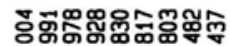

(1)

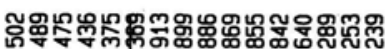

ส
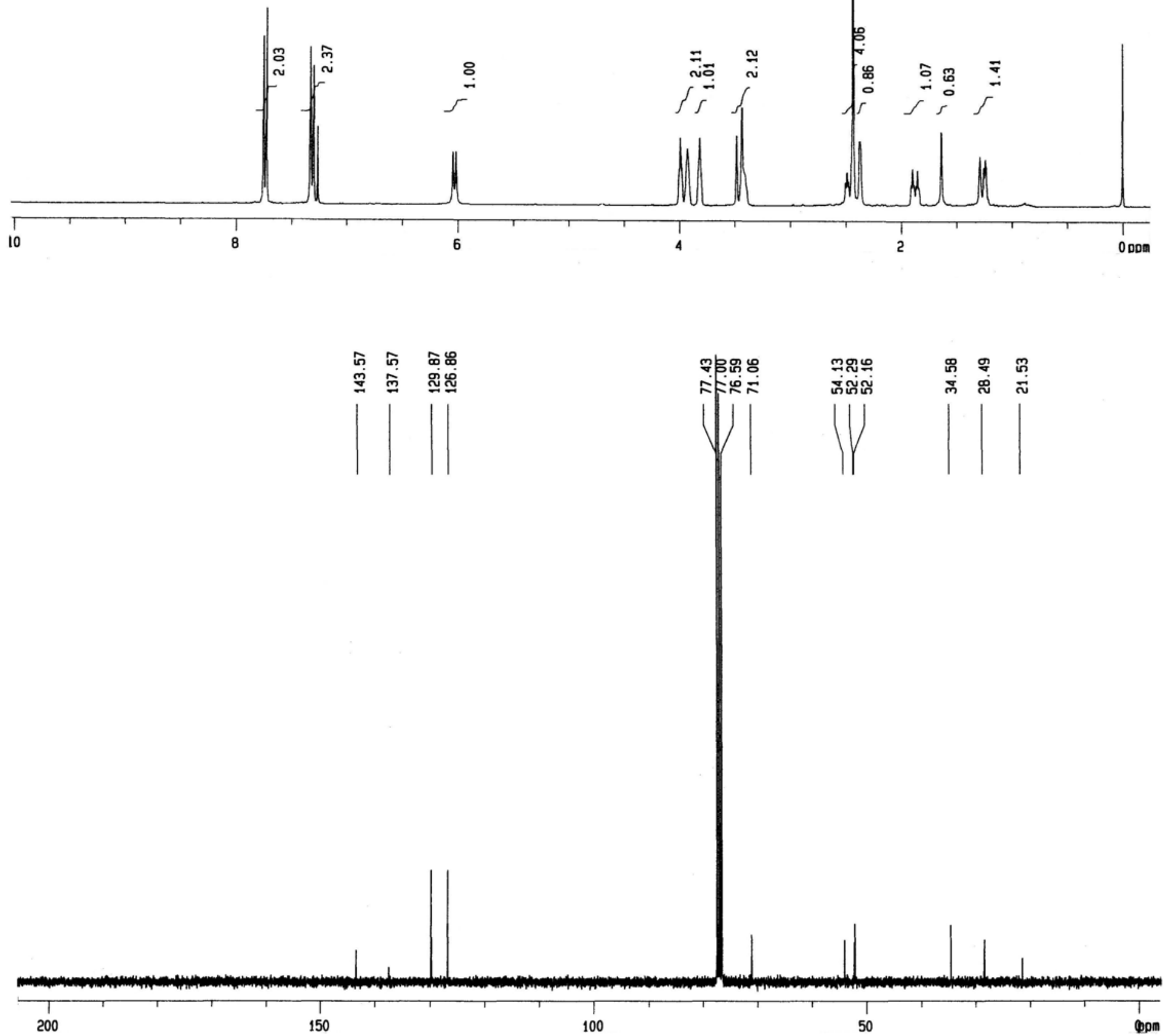

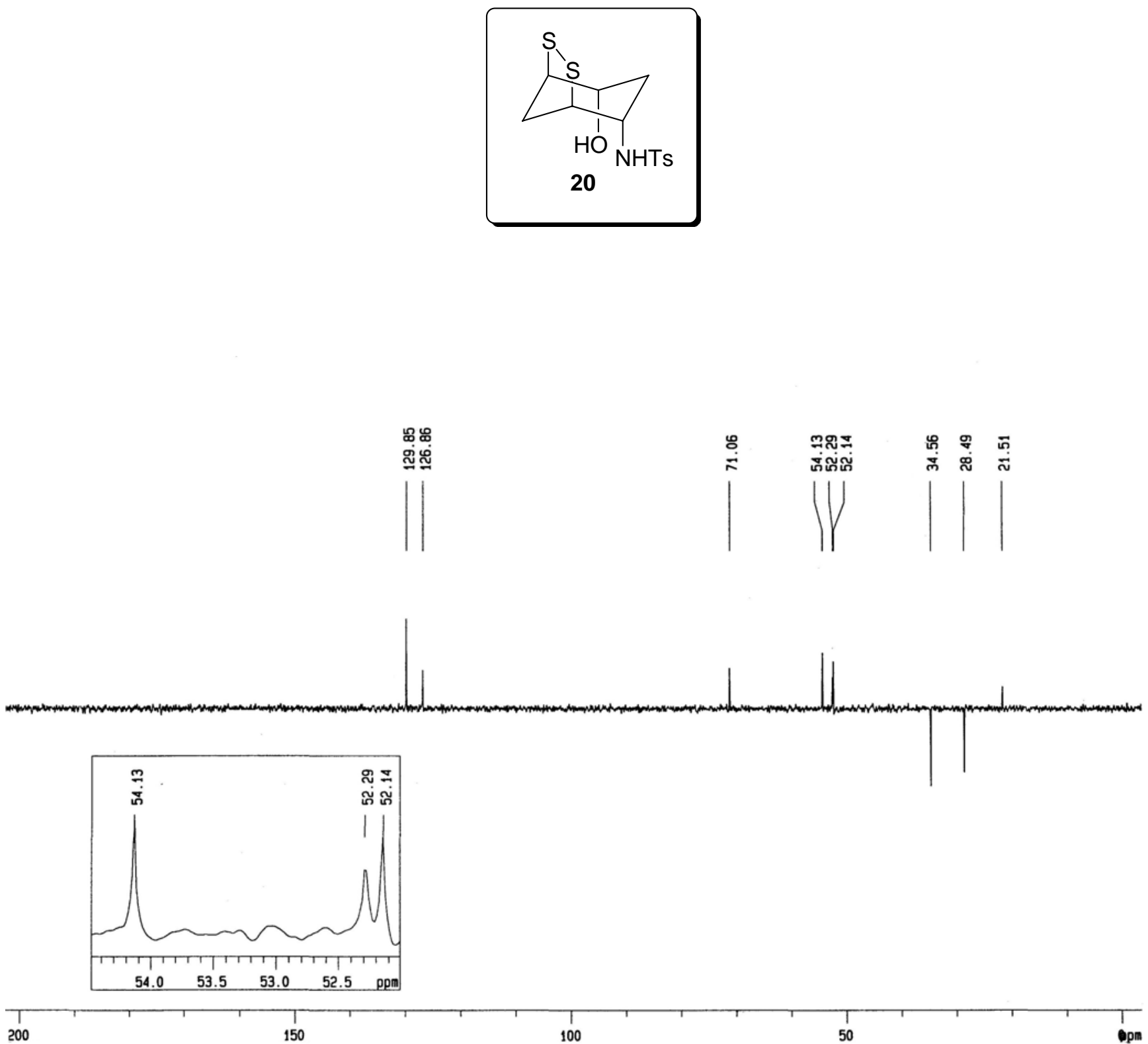

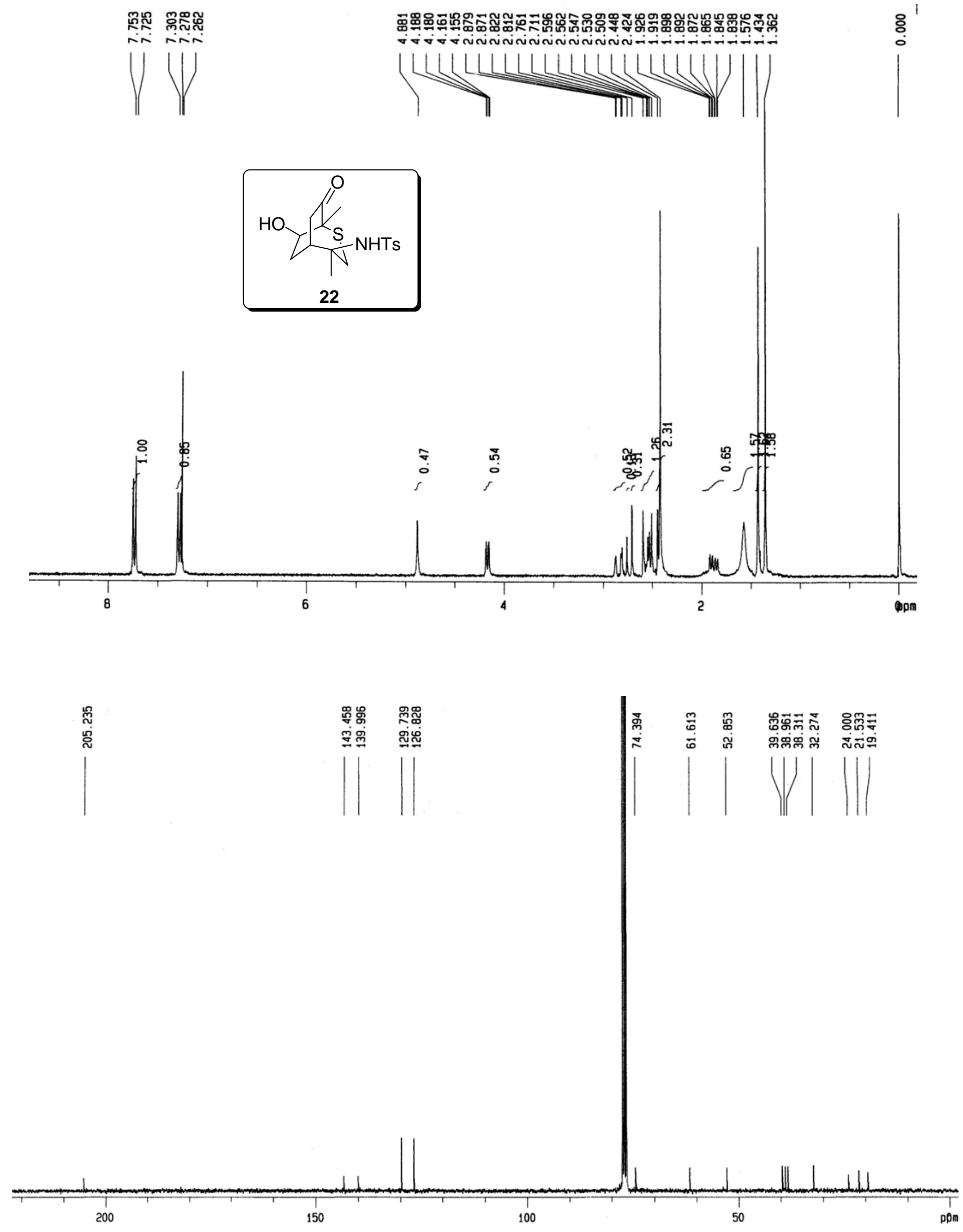


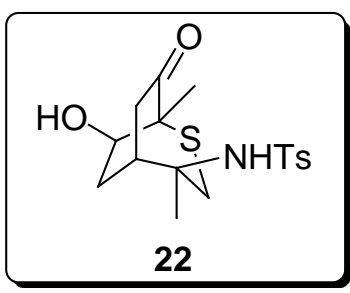

옹 品

誉

ฐ્ญำ

त

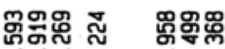

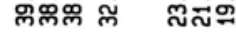
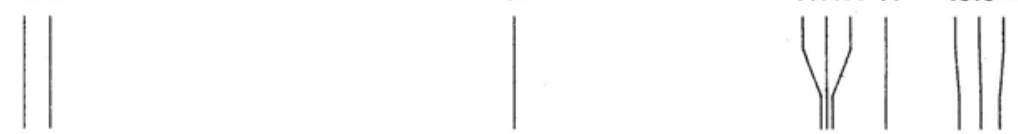

(1)

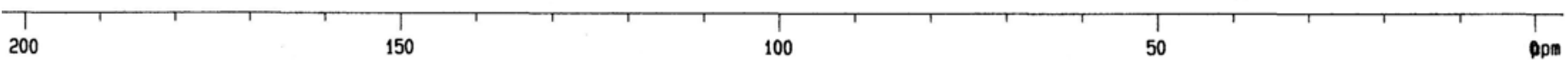



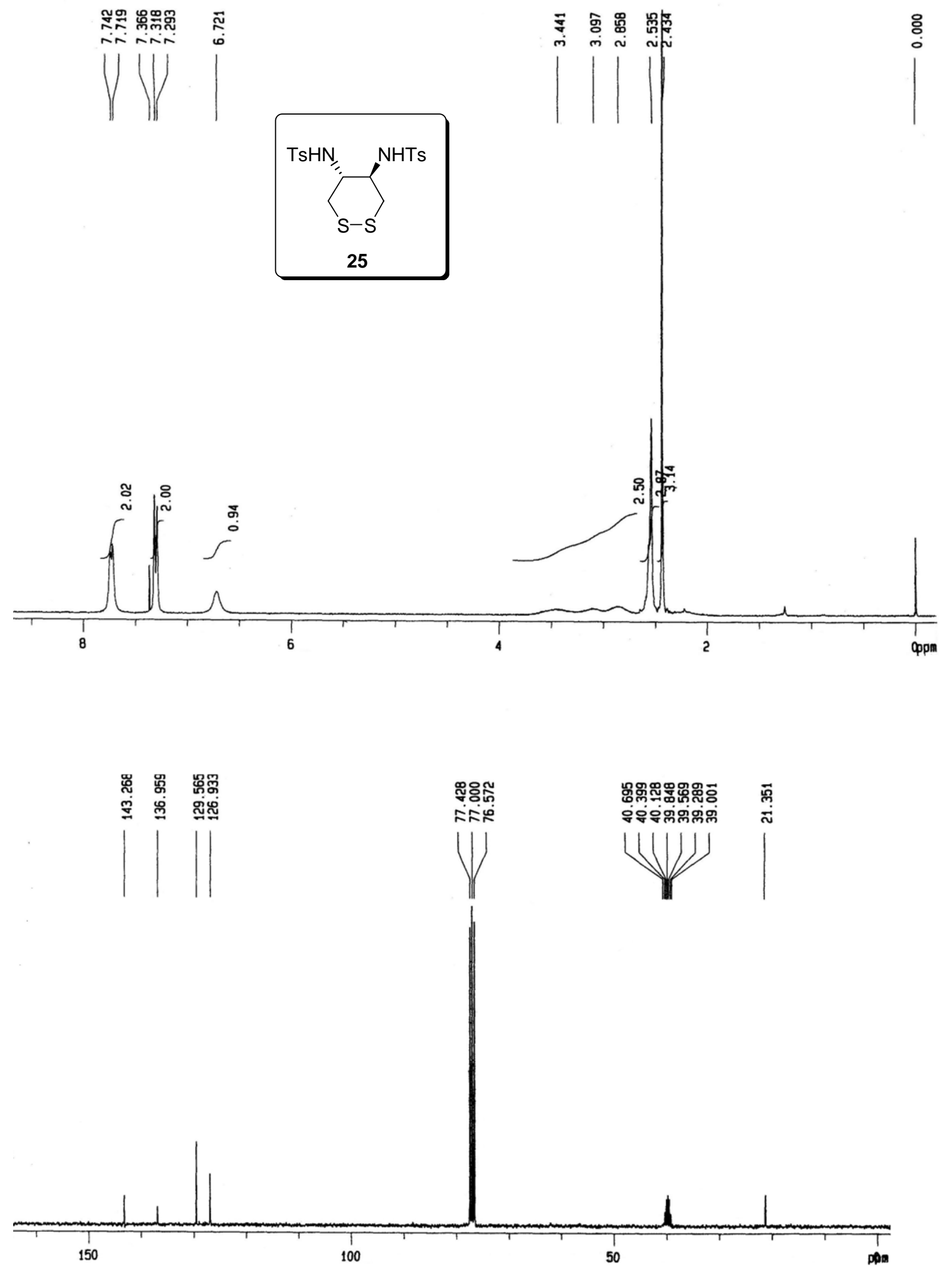


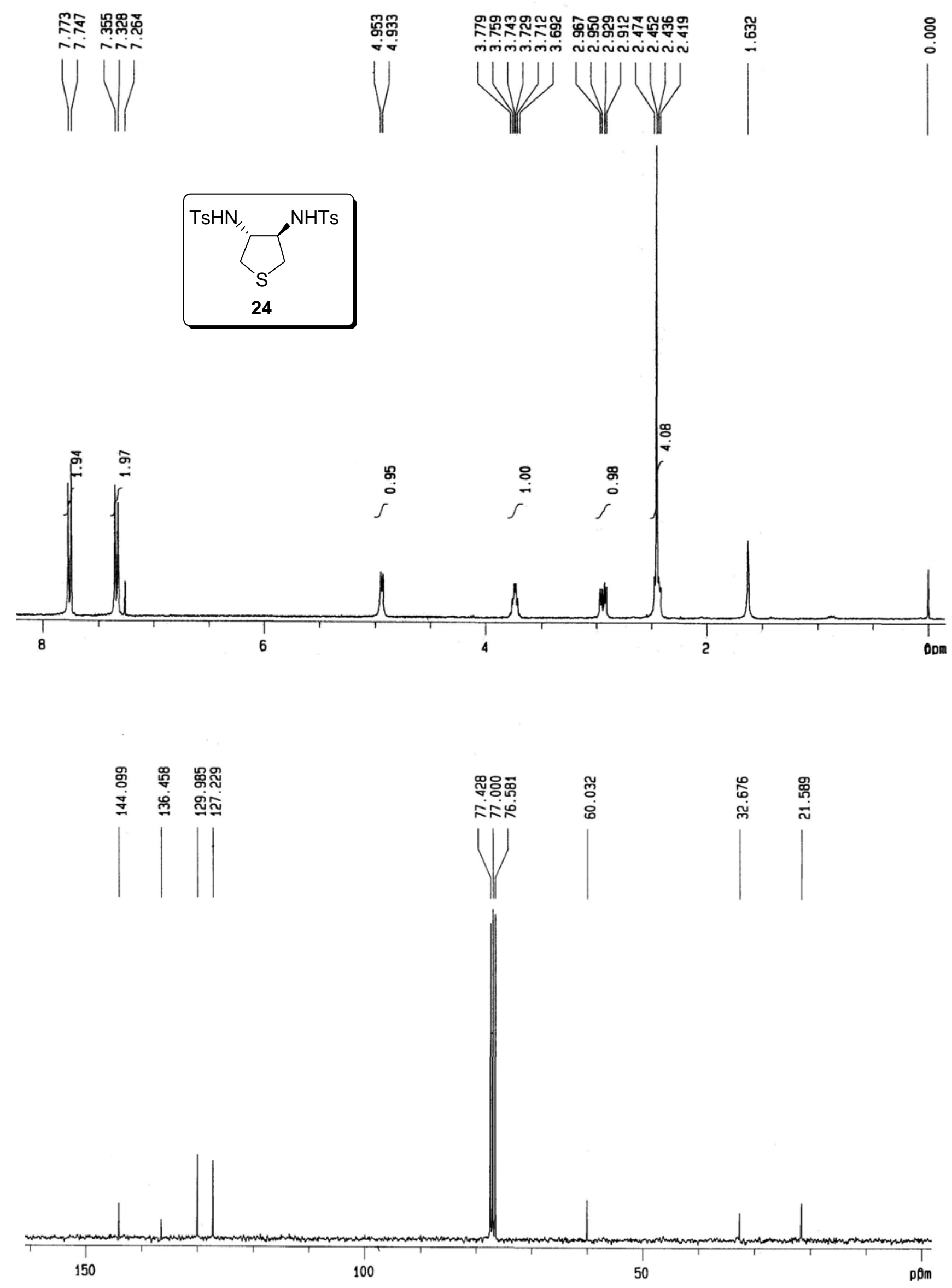



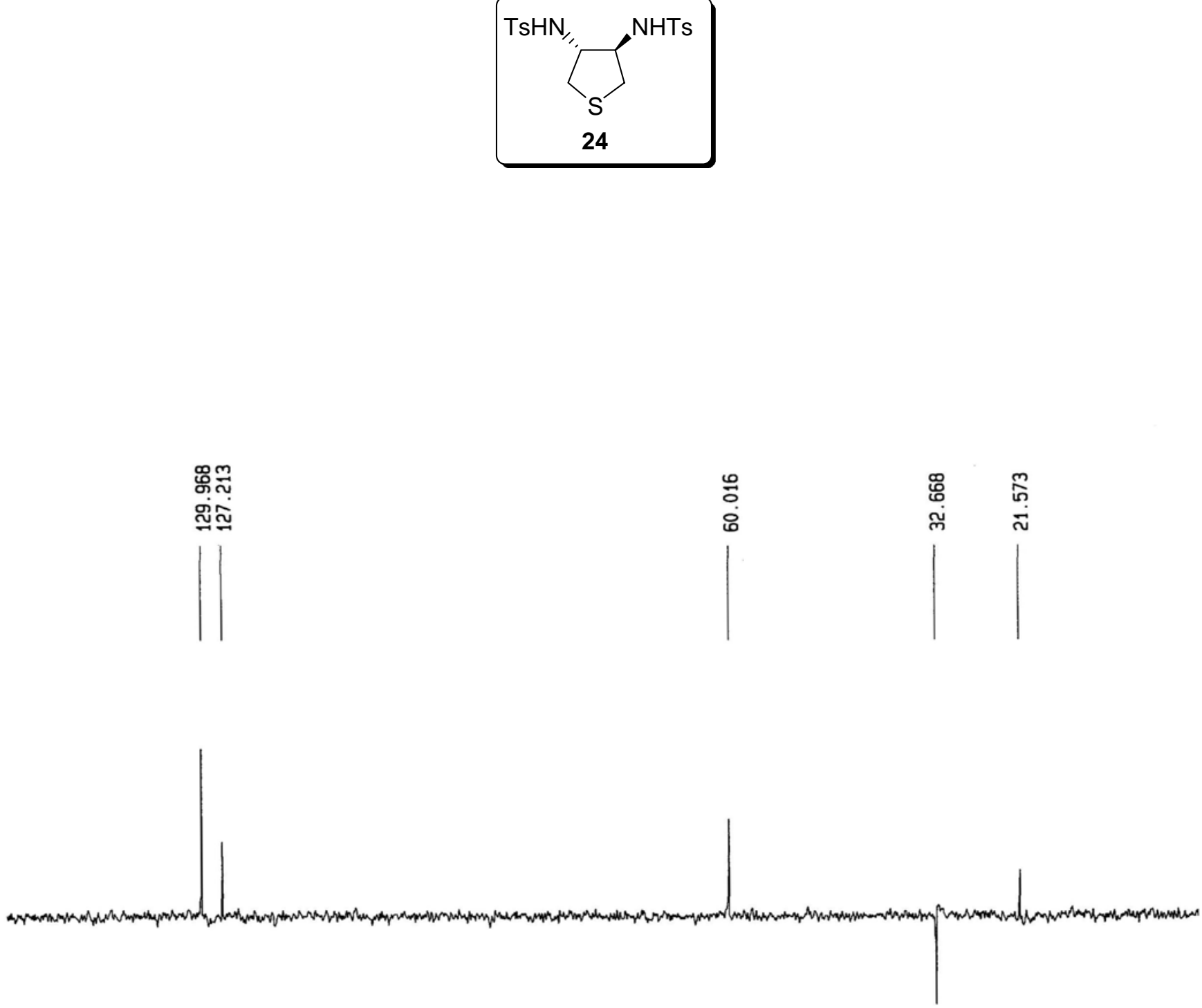

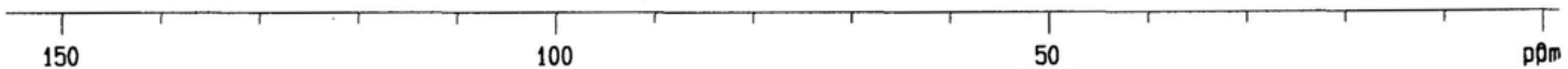



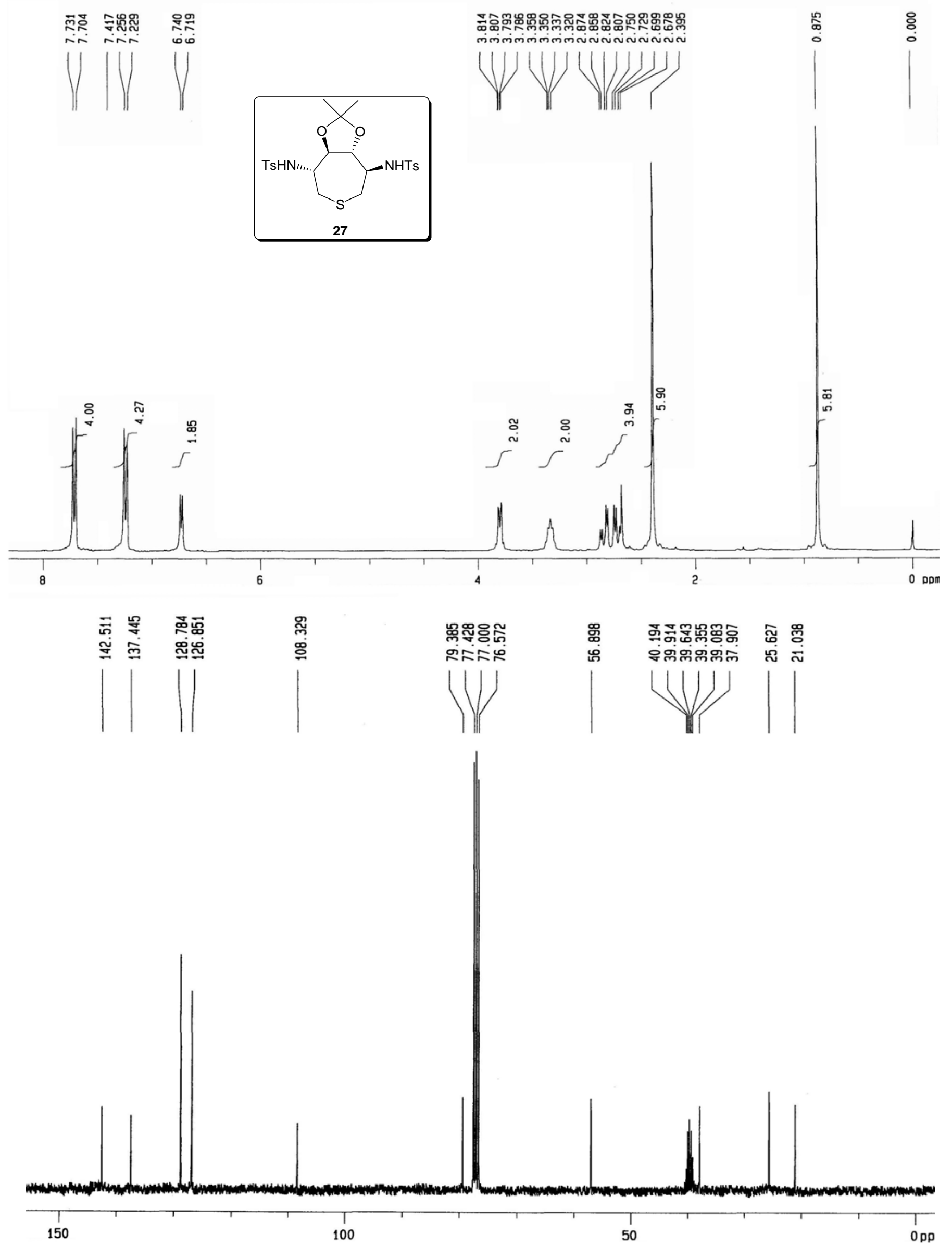

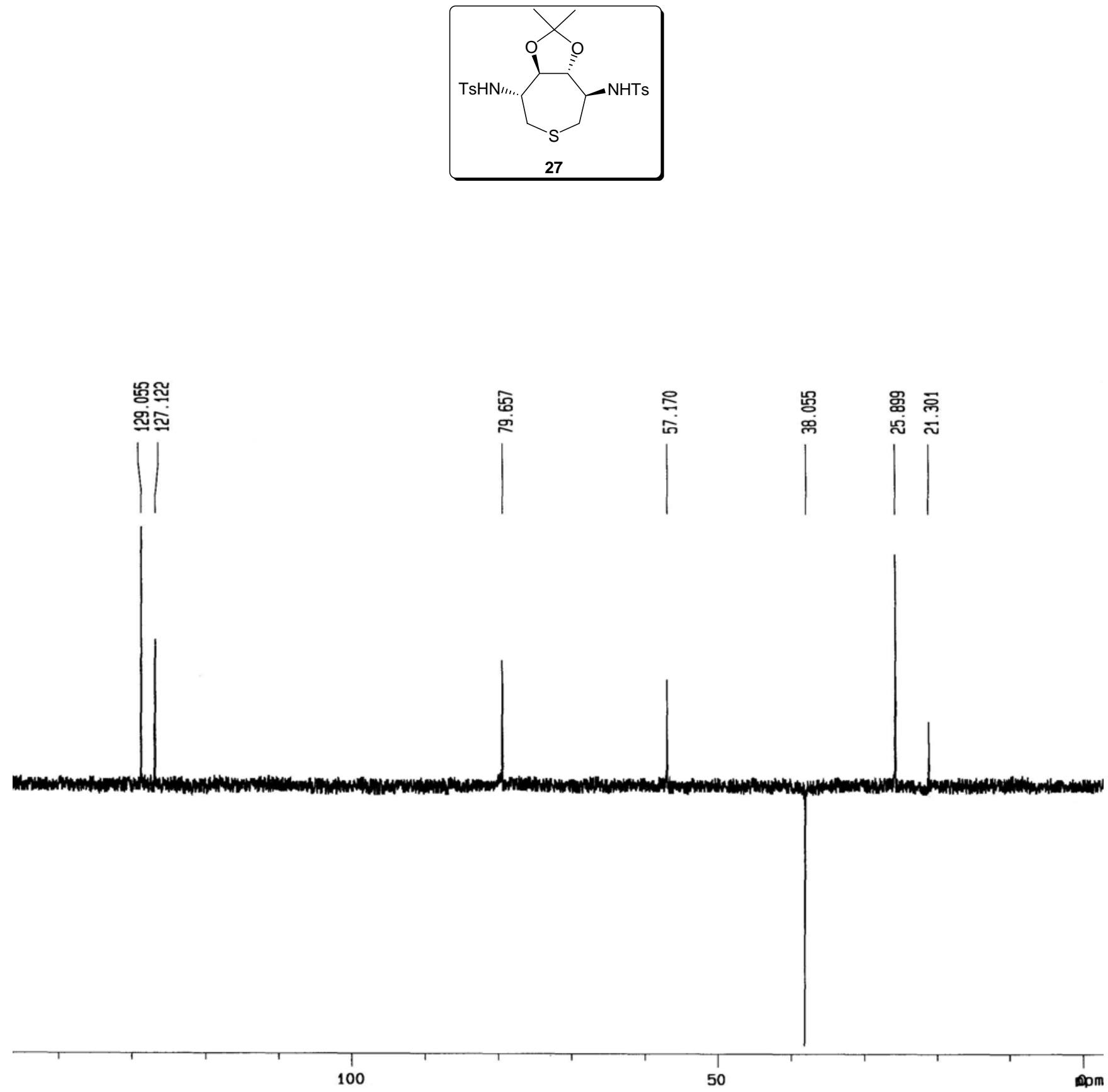